\title{
Space-Time Picture of Semiclassical Resonances
}

\author{
C. Gérard ${ }^{1}$ and I. M. Sigal ${ }^{2 \star, \star \star}$ \\ ${ }^{1}$ Centre de Mathématiques, Ecole Polytechnique, F-91128 Palaiseau Cedex, France \\ 2 Department of Mathematics, University of Toronto, Toronto M5S1A1, Canada
}

Received June 20, 1991

\begin{abstract}
This paper addresses the theory of quasiclassical resonances for Schrödinger operators with potentials smooth outside some possible local singularities. We introduce the notion of quasiresonance similar to that of quasimode, but incorporating a condition revealing its scattering nature, and describe its spacetime behaviour. The definition is given in terms of the original Schrödinger operator and uses a description of its frequency set. The result on the space-time behaviour justifies the intuitive picture of resonances as metastable states or "bound states with finite life-times." We demonstrate how quasiresonances arise in several natural situations.
\end{abstract}

\section{Introduction}

The quantum resonance is one of the central notions in Modern Physics. However, its mathematical understanding is still at a preliminary stage. The formal definition of the resonance in terms of the poles of a meromorphic continuation of the $S$-matrix or in terms of bumps in the scattering cross section, given in Physics, is hard to study. In Mathematical Physics the resonances are defined as complex eigenvalues of quantum Hamiltonians deformed by complex canonical transformations. This approach was developed in successive degrees of generality in [Ag-Co, $\mathrm{Ba}-\mathrm{Co}, \mathrm{Si} 2, \mathrm{~S} 1, \mathrm{Hu} 1, \mathrm{Cy}, \mathrm{He}-\mathrm{Sj} 2]$. One knows that both definitions yield the same object for two-body potentials (see for example [Ba1, Ge-Ma1]) and in some cases for $N$-body potentials (see [Ba2, Ha, De, S3]). Both definitions require analytic (either in the coordinate or momentum representation) potentials, use more complicated objects than original Hamiltonians and are not accessible for phase-space analysis ( $[\mathrm{He}-\mathrm{Sj} 2]$ uses a phase-space analysis but not in the original phase-space).

* Supported by NSERC under grant NA7901

$\star \star$ I.W.K. Killam Research Fellow 
The purpose of this paper is two-fold. First, we introduce the notion of quasiresonance. This notion is given directly in terms of the original quantum Hamiltonian, it is similar to that of a quasimode except that the square integrability is replaced by a condition pertaining to the propagation nature of the resonance. As in the case of quasimodes the main idea behind our definition is to neglect from the very beginning certain "inessential terms." As our analysis and examples show such an approach saves much of an effort. It allows to formulate the problem in terms of the Hamiltonians themselves and with natural restrictions on the potentials. Next we break up the unitary invariance of the conventional spectral theory by fixing a bounded region where the corresponding metastable will live for some time and an exterior region into which it will eventually escape.

Secondly, we describe the space-time behaviour of resonances corresponding to the intuitive picture of resonances as metastable states or "bound states with finite life-times" (see [Sk1, Sk2, Sk3, O, Hu2] for earlier results in this direction and [Si1] for a review). This result is an easy consequence of our definition. It determines the region in the phase-space in which the resonance states are essentially localized and their rate of escape from this region.

In this paper we deal with the quasiclassical regime which provides an additional structure necessary to define the resonance. We relate the notion of resonance to propagation of quasiclassical frequency set. The negligible terms are those of $O\left(h^{\infty}\right)$, where $h$ is a quasiclassical parameter (the Planck constant divided by $2 \pi$ ). Description of singularities modulo exponentially small terms would require analytic potentials and results on propagation of "analytic singularities." It is left out of this paper.

As an example we demonstrate that equilibrium points and hyperbolic orbits of the underlying classical system produce quasiresonances and compute the latter. These computations are rather standard exercises, in the second case it is analogous to the derivation of the Bohr-Sommerfeld quantization rules. The results coincide, of course, with earlier results of $[\mathrm{Ge}-\mathrm{Sj}, \mathrm{Sj}, \mathrm{B}-\mathrm{C}-\mathrm{D}]$ which were also given modulo $O\left(h^{\infty}\right)$ but whose derivations were rather involved.

In this paper we consider the Schrödinger operator

$$
P_{h}=-h^{2} \Delta+V(x)
$$

acting on $L^{2}\left(\mathbb{R}^{n}\right)$ with the quasiclassical parameter $h$. The quasiresonance states are defined as distributions $u_{h}$ solving the Schrödinger equation:

$$
P_{h} u_{h}-E(h) u_{h}=0 \bmod O\left(h^{\infty}\right)
$$

microlocally in some open set $\Omega$ of phase space. Here $E(h)$ is called a quasiresonance and we assume that $E(h) \rightarrow \lambda_{0 \in \mathbb{R}}$ when $h \rightarrow 0$.

In order to distinguish between incoming and outgoing resonances, we require that the quasiresonant state $u_{h}$ has singularities (i.e. frequency set, see Definition 3.1) only in a closed subset of phase space called the outgoing tail (see Definition 2.3). Let $p(x, \xi)=\xi^{2}+V(x)$ be the classical Hamiltonian function. The outgoing tail is the set of points $(x, \xi)$ in $p^{-1}\left(\lambda_{0}\right)$ such that the classical trajectory starting from $(x, \xi)$ does not go to infinity for negative times. In particular a quasiresonant state cannot have singularities at points $(x, \xi)$ which are trapped for positive times without being also trapped for negative times. We construct quasiresonant states and quasiresonances in all the cases where for dilation-analytic potentials true 
resonances have been computed in the semiclassical limit. Namely we consider in Sect. 4 quasiresonant states generated by a closed hyperbolic trajectory (treated in $[\mathrm{Ge}-\mathrm{Sj}]$ ), and by a potential maximum (treated in [B-C-D]), $[\mathrm{Sj}]$ ). Moreover if for example one considers a two-body Hamiltonian with a dilation-analytic potential, then it is easy to show that a resonant function is a quasiresonant state.

To construct metastable states out of quasiresonant states, we cut them of in a bounded region of phase space by applying a pseudodifferential operator $\chi\left(x, h D_{x}\right)$, where $\chi(x, \xi)$ is a smooth cutoff function. Then our main result can be described as follows (see Theorem 3.7):

$$
e^{-i t P_{h} / h} \chi\left(x, h D_{x}\right) u_{h}=e^{-i t E(h) / h} \chi\left(x, h D_{x}\right) u_{h}+r_{\text {out }}(t)+r_{\infty}(t),
$$

where $r_{\infty}(t)$ has a norm $O\left(h^{\infty}\right)$ uniformly for $t \geqq 0$ and $r_{\text {out }}(t)$ is an outgoing function in the sense that:

$$
\left\|\chi_{0}(x) r_{\text {out }}(t)\right\|=\frac{O\left(h^{\infty}\right)}{\langle t\rangle^{\infty}} .
$$

Here $\chi_{0}(x)$ is a suitable cutoff function. One also gets a sharper estimate on $r_{\text {out }}(t)$ (see Theorem 3.8) which essentially shows that $r_{\text {out }}(t)$ propagates out with a velocity bounded from below. These estimates hold true provided $\operatorname{Im} E(h)$ is not $O\left(h^{\infty}\right)$. We then study time decay in a situation where this condition is not satisfied, namely the well known shape resonances. In this case we need to assume that the potential is two-body and dilation-analytic. It is then well known (see for example [C-D-K-S, $\mathrm{He}-\mathrm{Sj} 2, \mathrm{Hi}-\mathrm{Si}]$,) that the width $\Gamma(h)$ of the shape resonances is $\widetilde{O}\left(e^{-2 S_{0} / h}\right)$, where $S_{0}$ is some Agmon distance and $\widetilde{O}(a)$ means $O_{\varepsilon}\left(a e^{\varepsilon / h}\right)$ for any $\varepsilon$. Then if $u_{h}$ is a resonant function associated with a resonance $E(h)$ we have the following time decay estimate:

$$
e^{-i t P_{h} / h} \chi\left(x, h D_{x}\right) u_{h}=e^{-i t E(h) / h} \chi\left(x, h D_{x}\right) u_{h}+r_{\text {out }}(t)+r_{\infty}(t),
$$

where:

$$
\left\|r_{\infty}(t)\right\|=O\left(e^{-S_{0} / h}\right)+\widetilde{O}\left(e^{-2 S_{0} / h}\right)\left(\frac{h}{\Gamma(h)}\left(1-e^{-t \Gamma(h) / h}\right)\right)
$$

uniformly for $t \geqq 0$ and $r_{\text {out }}(t)$ is an outgoing function in the sense that:

$$
\left\|\chi_{0}(x) r_{\text {out }}(t)\right\|=O\left(h^{\infty} e^{-S_{0} / h}\right)\left(e^{-t \Gamma(h) / h}+\langle t\rangle^{-\infty}\right)
$$

uniformly for $t \geqq 0$. (See Theorem 4.3). The estimate on $r_{\infty}(t)$ shows that our result is meaningful as long as $t$ is less than $C e^{-\varepsilon_{0} / h} \Gamma(h)$ for some $\varepsilon_{0}>0$. This difference between resonances created by tunneling and others may come from the fact that the decoupling between the decaying part of the metastable state and the outgoing part is caused here by ellipticity instead of propagation.

Let us now say a word about the techniques used in this paper. The crucial point is the dependence in $t$ of the constants arising for example in (1.3), (1.5). For example using a very simple argument of propagation of frequency set for solutions of pseudodifferential equations one gets that for finite time $t$ one has $\left\|\chi_{0}(x) r_{\text {out }}(t)\right\|=O_{t}\left(h^{\infty}\right)$, but without nice control on the size of $O_{t}$.

In order to have good estimates with respect to parameter $t$, we use the method of propagation observables introduced by Sigal-Soffer in [S-S]. An important 
intermediate result is a semiclassical version of the propagation estimate of $[\mathrm{S}-\mathrm{S}]$ (see Theorem 6.1). The essential idea (familiar also in partial differential equations) is to construct an operator $F_{t}$ such that the Heisenberg derivative $D_{t} F=$ $\partial_{t} F+i h^{-1}\left[P_{h}, F\right]$ is positive. The point is that one constructs $F_{t}$ essentially as a function $\chi(A / t)$ for some self adjoint operator $A$ and some cutoff function $\chi$. It seems that these operators are not in general pseudodifferential operators since the operators $A$ used (typically $\left.A=\left(x \cdot h D_{x}+h D_{x} \cdot x\right)\right)$ are not elliptic.

However there are two tools which allow to use this kind of operators almost as if they were pseudodifferential operators. The first one is a commutator expansion lemma whose use in this field is due to Sigal-Soffer (see Proposition 5.1) and the second a localization lemma (see Proposition 5.2) which is used to compose these operators with pseudodifferential cutoffs. We think that these methods could be of interest in other semiclassical problems.

The paper is organized as follows. In Sect. 2, we introduce the class of Hamiltonians we are going to consider in this paper and we give some examples. In Sect. 3, we introduce the definition of quasiresonance states and prove the main results of this paper, namely Theorems 3.7 and 3.8, using results from Sects. 5,6,7. Section 4 is devoted to the construction of quasiresonant states created by a closed trajectory of hyperbolic type and by a potential maximum (barrier top resonances). We also study time decay of cutoffs of resonant states for the shape resonance problem. We assume here that the potential is two-body and dilation-analytic (see Theorem 4.3). In Sect. 6 we prove a semiclassical version of the abstract propagation estimate of Sigal-Soffer and apply it to our class of Hamiltonians. Finally in Sect. 7, we prove minimal velocity estimates in the spirit of Sigal-Soffer. Some auxiliary results are given in the Appendix.

Notations. In all the paper, we will denote by $H^{s}(\Omega)$, the usual Sobolev space of order $s$ on an open set $\Omega \in \mathbb{R}^{n}$, and by $\|\cdot\|$ the $L^{2}$ norm on $\mathbb{R}^{n}$. For given hamiltonian $H$ and vector $u$, we will denote by $\langle A\rangle$ the (time-dependent) expectation value of $A$ given by $\left\langle A e^{-i t H} u, e^{-i t H} u\right\rangle$.

\section{Hamiltonians and Escape Functions}

In this section we introduce the class of Hamiltonians which will be studied in this paper. We also recall some geometric consequences of the existence of suitable escape functions. In all the paper, we will denote the function $\left(1+x^{2}\right)^{1 / 2}$ by $\langle x\rangle$.

2.A. Hypotheses on the Hamiltonian. We will consider semiclassical Schrödinger operators $P_{h}=-h^{2} \Delta+V(x)$, on $L^{2}\left(\mathbb{R}^{n}\right)$, where the potential $V=V_{r}+V_{s}$ satisfies the following conditions:

(H.1i): $V_{s}$ is a compactly supported multiplicative potential such that $V_{s}$ is $\Delta$ bounded with relative bound strictly less than one.

(H.1ii): $V_{r}$ is a smooth real potential satisfying some symbol type estimates.

To describe these estimates in a compact form, we will use the framework of the Weyl calculus for which we refer to the book of Hörmander [Hö]. Let $g_{x}(d x)$ be a metric on $\mathbb{R}^{n}$. We require that $V_{r}$ belongs to the symbol class $S\left(1, g_{x}\right)$, i.e. that $V_{r}$ satisfy the following estimates:

$$
\forall k \in \mathbb{N}, \quad \sup _{t_{i} T^{*}\left(\mathbb{R}^{n)}\right.}\left|V^{(k)}\left(x, t_{1}, \ldots, t_{k}\right)\right| \prod_{1}^{k} g_{x}\left(t_{i}\right)^{-1 / 2} \leqq C_{k},
$$


where $V^{(k)}$ is the $k^{\text {th }}$ differential of $V$. We will denote by $p=\xi^{2}+V_{r}(x)$ the symbol of $-h^{2} \Delta+V_{r}$, which is the regular part of $P_{h}$. The symbol $p$ belongs to the symbol class $S\left(\langle\xi\rangle^{2}, g\right)$, where $g$ is the metric on $T^{*}\left(\mathbb{R}^{n}\right)$ defined by:

$$
g_{x, \xi}(d x, d \xi)=\frac{(d \xi)^{2}}{\langle\xi\rangle^{2}}+g_{x}(d x) .
$$

To have a good symbolic calculus in the class $S\left(\langle\xi\rangle^{2}, g\right)$, we will make the following hypotheses on $g_{x}$ :

(H.2): $g_{x}$ is slowly varying i.e.:

$\exists \varepsilon$ and $C$ such that if $g_{x}(x-y) \leqq \varepsilon$, then $\forall d x \in \mathbb{R}^{n}, C^{-1} g_{x}(d x) \leqq g_{y}(d x) \leqq C g_{x}(d x)$,

(H.3): $g_{x}$ is $\sigma$-temperate which in our case is equivalent to:

$$
\exists C, N \text { such that } \forall t \in \mathbb{R}^{n} g_{y}(t) \leqq C g_{x}(t)\left(\langle x-y\rangle^{N}\right),
$$

(H.4): $g_{x}(d x) \leqq(d x)^{2}$ and $g_{x}(x) \leqq 1$.

As an immediate consequence of hypotheses (H.1) and (H.2), we see that $P_{h}$ is self-adjoint with domain $H^{2}\left(\mathbb{R}^{n}\right)$.

2.B. Hypotheses on the Escape Function. As we mentioned in the Introduction, in order to define resonances near a given energy level $\lambda$, one needs to have some control on the behaviour of the Hamiltonian near infinity. In semiclassical problems, this control can be obtained by making hypotheses on the classical Hamiltonian near infinity. We formulate these hypotheses by introducing an escape function and the so-called non-trapping condition. This notion of escape function was first introduced in the context of semiclassical resonances by Helffer and Sjöstrand (see [He-Sj2]). We start by introducing the following definition:

Definition 2.1. An energy interval I is called non-trapping for $p$ if $\forall(x, \xi) \in p^{-1}(I)$, one has: $\exp t H_{p}(x, \xi) \rightarrow \infty$ when $t$ tends either to $+\infty$ or to $-\infty$. Here $H_{p}$ denotes the Hamiltonian vector field of $p$.

Our first hypothesis is a condition ensuring that the classical Hamiltonian $p$ has no trapped trajectories near infinity. So we ask that there exist a function $G(x, \xi)$ in $C^{\infty}\left(T^{*}\left(\mathbb{R}^{n}\right)\right)$ called an escape function such that: $S(1, g)$.

(H.5): $G$ is of the form $G=\langle x, \xi\rangle+r(x, \xi)$, where $r$ belongs to the symbol class

(H.6): there exists an energy interval $I$ such that:

$$
H_{p} G \geqq C_{0} \quad \text { on } \quad p^{-1}(I) \cap\{|x| \geqq R\} \quad \text { for } \quad C_{0}>0, R \gg 1 .
$$

(H.7): there exist a real function $W \in C_{0}^{\infty}\left(\mathbb{R}^{n}\right)$ such that $I$ is non-trapping for $p_{1}=p+W$.

Let us now recall for later use two results proven in [Ge-Sj] and [ $\mathrm{Ge}-\mathrm{Ma} 2]$.

Proposition 2.2. i) Under hypotheses (H.5), (H.6) there exists a function $r_{0} \in C_{0}^{\infty}\left(T^{*}\left(\mathbb{R}^{n}\right)\right)$ such that:

$$
H_{p}\left(G+r_{0}\right) \geqq 0 \quad \text { on } \quad p^{-1}(I) .
$$

ii) Under hypotheses (H.6), if $I$ is non-trapping for $p$, then there exists a function $r_{1} \in C_{0}^{\infty}\left(T^{*}\left(\mathbb{R}^{n}\right)\right)$ such that if $G_{1}=G+r_{1}$, one has:

$$
H_{p} G_{1} \geqq C_{0} \quad \text { on } p^{-1}(I) \text { for } C_{0}>0 .
$$


We consider now the trapped part of the phase-space. We recall a geometric definition from [Ge-Sj, Appendix].

Definition 2.3. The outgoing (respectively incoming) tail $\Gamma_{ \pm}$for an energy interval $I$ is the set

$$
\left\{(x, \xi) \in p^{-1}(I) \mid \exp t H_{p}(x, \xi) \nrightarrow \infty, t \rightarrow \mp \infty\right\} .
$$

The trapped set is defined as $K=\Gamma_{+} \cap \Gamma_{-}$.

Note that from the existence of $G(x, \xi)$, we know that $K$ is a compact set in $p^{-1}(I)$.

2.C. Examples. We now give some examples of potentials satisfying our hypotheses.

Example 1: Two-body Potentials. These are potentials for which:

$$
\left|D_{x}^{\alpha} V_{r}(x)\right| \leqq C_{\alpha}\langle x\rangle^{-\rho-|\alpha|} \text { for } \rho>0, \quad \alpha \in \mathbb{N}^{n} .
$$

Then $V=V_{s}+V_{r}$ satisfies the hypotheses $(H)$ with the escape function $G(x, \xi)=\langle x, \xi\rangle$ on any bounded interval $I$ included in $\mathbb{R}_{+}^{*}$.

Example 2: $N$-body Potentials. These are potentials whose behaviour at infinity varies depending on the direction. Let $V_{s}=0$ and $V=V_{r}$ be given by:

$$
V(x)=\sum_{a \in \mathscr{A}} V_{a}\left(\pi^{a} x\right)
$$

Here $\mathscr{A}$ is a set labeling a family of vector subspaces $X^{a}$ and $\pi^{a}$ are orthogonal projections on $X^{a}$. Assume that each potential $V_{a}$ satisfies the estimates (2.2) on $X^{a}$. Then $V$ satisfies the hypotheses $(H)$ with an escape function $G=\langle x, \xi\rangle+r(x, \xi)$ for some function $r \in S(1, g)$ on a bounded energy interval $I$, if $I$ is non-trapping for all classical subhamiltonians. We refer to the Appendix 1 for a description of the metric $g$ and for detailed proofs.

\section{Resonances and Metastable States}

In this section we shall introduce the definition of quasiresonance states and quasiresonances for Hamiltonian considered in Sect. 2. These quasiresonant states are analogues of the well-known quasimodes in situations where discrete spectrum appears. We will then prove time decay estimates for the quantum evolution of these states which exhibit their metastable behaviour. Finally as an illustration of the influence of trapped trajectories for the classical system, we prove some propagation estimates for the non-trapping case.

3.A. The Frequency Set. To formulate the notion of a quasiresonance state, we will first recall the definition of the frequency set of an $h$-dependent distribution (see Guillemin-Sternberg [Gu-St]).

Definition 3.1. Let $u(x, h) \in D^{\prime}\left(\mathbb{R}^{n}\right)$ be a distribution such that for any open set $\Omega$, there exist $N_{0} \in \mathbb{N}$ with $\|u(x, h)\|_{H^{-N_{0}(\Omega)}}=O\left(h^{-N_{0}}\right)$. Then a point $\left(x_{0}, \xi_{0}\right)$ in $T^{*}\left(\mathbb{R}^{n}\right)$ is said not to belong the frequency set of $u$, denoted by $F S_{h} u$, if there exists a cutoff function $\chi(x, \xi)$ with $\chi\left(x_{0}, \xi_{0}\right) \neq 0$ and:

$$
\left\|\chi\left(x, h D_{x}\right) u\right\|=O\left(h^{\infty}\right) .
$$


We now recall the fundamental result of propagation of $F S_{h} u$ (see [Ro]).

Theorem 3.2. Let $p(x, \xi)$ be a real symbol in $S\left(m, d x^{2}+d \xi^{2}\right)$ with a weight $m$, such that $d p \neq 0$. Then $F S_{h} u \backslash F S_{h}\left(p\left(x, h D_{x}\right) u\right)$ is a union of integral curves of $H_{p}$.

A consequence of Theorem 3.2 adapted to our problem is:

Corollary 3.3. Let $V$ be smooth and real. Let $u_{0}(x, h)$ be an $L^{2}$ function with $\left\|u_{0}\right\|_{L^{2}}=O\left(h^{-N_{0}}\right)$ for some $N_{0} \in \mathbb{N}$. Then if $u_{t}=e^{-i t P_{h} / h} u_{0}$, one has:

$$
\left(x_{0}, \xi_{0}\right) \in F S_{h} u_{0} \Leftrightarrow\left(x_{t}, \xi_{t}\right) \in F S_{h} u_{t},
$$

where $\left(x_{t}, \xi_{t}\right)=\exp t H_{p}\left(x_{0}, \xi_{0}\right)$.

3.B. Quasiresonance States. We now introduce the definition of quasiresonances states. We consider a semiclassical Schrödinger Hamiltonian $P_{h}=-h^{2} \Delta+V(x)$, where $V$ satisfies the hypotheses $(H)$ of Sect. 2. It follows from Hypothesis $(H .5)$ that there exists constants $c_{0}$ and $R$ such that $H_{p} G \geqq c_{0}$ on $p^{-1}(I) \cap\{|x| \geqq R\}$, where $I$ is a given energy interval and $R$ can be chosen such that the support of the singular potential $V_{s}$ is included in $\{|x| \leqq R-1\}$.

Definition 3.4. An h-dependent distribution $u_{h}(x) \in H^{2}\left(\mathbb{R}^{n}\right)$ is called a quasiresonance state associated with the quasiresonance $E(h)$ if :

i) $\forall R_{0} \geqq 1, \exists N$ such that $\left\|u_{h}\right\|_{H^{-N}\left(\left\{|x| \leqq R_{0}\right\}\right)}=O\left(h^{-N}\right)$.

ii) There exists a compact set $K_{0} \subset \mathbb{R}^{n}$ such that $\left\|u_{h}\right\|_{L^{2}\left(K_{0}\right)} \geqq 1$.

iii) $\left(P_{h}-E(h)\right) u_{h}=O\left(h^{\infty}\right)$ in $L_{\text {loc }}^{2}\left(\mathbb{R}^{n}\right)$.

iv) $u_{h}$ is outgoing near infinity i.e.: there exists $C_{0}$ and $R_{0}$ such that:

$$
F S u_{h} \cap p^{-1}(I) \cap\left\{|x| \geqq R_{0}\right\} \subset\left\{(x, \xi) \mid G(x, \xi) \geqq C_{0}\right\} .
$$

v) $E(h) \rightarrow \lambda_{0}$, for some $\lambda_{0} \in \dot{I}$.

Let us first make some comments on this definition.

Remark 1. It follows easily from iii) and v) that $F S u_{h} \cap\{|x| \geqq R\} \subset p^{-1}\left(\lambda_{0}\right)$, if $R$ is chosen such that $\operatorname{supp} V_{s} \subset\{|x| \leqq R-1\}$.

Remark 2. If the frequency set of $u_{h}$ does not intersect the singular support of $V_{s}$ (which means that the singularities of the potential have no influence on the quasiresonance state), one can replace condition iv) by simpler conditions. This remark is expanded in the next proposition.

Proposition 3.5. Let $u_{h}$ be a quasiresonant state. Then $u_{h}$ the following properties:

$$
\forall C_{1}>0 \exists R_{1} \quad \text { such that } F S u_{h} \cap\left\{|x| \geqq R_{1}\right\} \subset\left\{(x, \xi) \mid G(x, \xi) \geqq C_{1}\right\} .
$$

If moreover $F S u_{h} \cap \operatorname{singsupp} V_{s}=\varnothing$, then the condition iv) is equivalent to any of the following conditions:

iv') $F S u_{h} \subset \Gamma_{+}$,

iv") for some $C_{1} \gg 1 F S u_{h} \cap\left\{(x, \xi) \mid G(x, \xi) \leqq-C_{1}\right\}=\varnothing$.

For the proof of Proposition 3.5, we refer to Appendix 3, Lemma C.1.

Remark 3. From Definition 3.4, it is clear that if $E(h)$ is a quasiresonance, $\operatorname{Im} E(h)$ is defined only modulo $O\left(h^{\infty}\right)$. In Sect. 4 we will see that under stronger hypotheses 
one can get also time decay estimates in the case of shape resonances where $\operatorname{Im} E(h)=O\left(e^{-C / h}\right)$ for some constant $C$.

3.C. Time Decay for Quasiresonance States. Our goal in this subsection is to prove time decay results from the evolution of a quasiresonant state $u_{h}$. In order to apply $e^{-i t P_{h} / h}$ to $u_{h}$ we first need to cut $u_{h}$ microlocally in a bounded region of phase space to turn it into an $L^{2}$ function. The cutoff region has to be large enough in order to eliminate the influence of the singular part of the potential and of the possible existence of trapped trajectories in a compact region of $p^{-1}(I)$. So we start by picking up a cutoff function in a convenient way and by introducing some notations. Let:

$$
C_{1}=1+\sup _{\operatorname{supp} W \cap p_{1}^{-1}(I)} G
$$

Here $W(x)$ is the modifying potential (see $(H .7)$ ) such that $I$ is non-trapping for $p_{1}=p+W$. We take then a cutoff function $\chi(x, \xi) \in C_{0}^{\infty}\left(T^{*}\left(\mathbb{R}^{n}\right)\right)$ such that:

$$
\operatorname{supp} \nabla \chi \subset\left\{(x, \xi) \||x| \geqq R_{1}\right\},
$$

where $R_{1}$ is large enough such that $\operatorname{supp} V_{s} \cup \operatorname{supp} W \subset\left\{|x| \leqq R_{1}-1\right\}$ and such that (3.1) holds with the constant $C_{1}$ chosen above. Let now introduce a definition:

Definition 3.6. $A$ cutoff function $\chi_{0}(x) \in C_{0}^{\infty}\left(\mathbb{R}^{n}\right)$ is adapted to $\left(C_{1}, R_{1}, I\right)$, if the following condition is fulfilled:

for any $(x, \xi)$ such that $G(x, \xi) \geqq C_{1}, \quad|x| \geqq R_{1}, \quad$ and $\quad p_{1}(x, \xi) \in I$, one has:

$$
\pi_{x}\left(\exp t H_{p_{1}}(x, \xi)\right) \notin \operatorname{supp} \chi_{0}, \quad \text { for } t \geqq 0 .
$$

It is easy to see using the fact that $I$ is non trapping for $p_{1}$ and Proposition 2.2 that one can find cutoffs adapted to $\left(C_{1}, R_{1}, I\right)$ with arbitrary large supports by taking $R_{1}$ large enough.

The following Theorem is our main result on time decay:

Theorem 3.7. Let $u_{h}$ be a quasiresonant state with quasiresonance $E(h)$ and let us denote by $a(h)=h^{-1} \operatorname{Im} E(h)$ the inverse of the lifetime of the quasiresonance $E(h)$. Then if $\chi(x, \xi)$ is a cutoff function as above we have:

$$
e^{-i t P_{h} / h} \chi\left(x, h D_{x}\right) u_{h}=e^{-i t E(h) / h} \chi\left(x, h D_{x}\right) u_{h}+r_{\text {out }}(t, h)+r_{\infty}(t, h)
$$

where:

i) $r_{\infty}(t, h)$ has an $L^{2}$ norm of size $O\left(h^{\infty}\right) a^{-1}\left(1-e^{-t a}\right)$ uniformly for $t \geqq 0$.

ii) if $\chi_{0}$ is an adapted cutoff, $r_{\text {out }}(t, h)$ satisfies the estimate:

$$
\left\|\chi_{0}(x) r_{\text {out }}(t, h)\right\|=O\left(h^{\infty}\right)\left(\frac{1}{a\langle t\rangle^{\infty}}+e^{-t a}\right), \text { uniformly for } t \geqq 0 .
$$

Let us make some comments on this theorem.

Remark 4. If $|\operatorname{Im} E(h)| \geqq C h^{N_{0}}$ for some $N_{0} \geqq 0$, then the estimates i), ii) become:

$$
r_{\infty}(t, h)=O\left(h^{\infty}\right), \quad \chi_{0}(x) r_{\text {out }}(t, h)=\frac{O\left(h^{\infty}\right)}{\langle t\rangle^{\infty}} .
$$


The estimate ii) captures the outgoing nature of $r_{\text {rout }}(t, h)$. To describe more precisely this outgoing behavior we prove the following result:

Theorem 3.8. There exist $v>0, c>0, T_{0} \geqq 1$ such that for any $0<\alpha<1$ we can write:

$$
r_{\text {out }}(t, h)=r_{\alpha}(t, h)+r_{\text {out }, \alpha}(t, h)
$$

with $r_{\alpha}$ and $r_{\text {out }, \alpha}$ satisfying:

i) if $a(h)=O_{h}(1)$, then:

$$
\left\|r_{\alpha}(t, h)\right\|=O\left(e^{-a t(1-\alpha)}\left(1+a(h)^{-1 / 2}\left(1-e^{-c a t}\right)^{1 / 2}\right)\right)
$$

uniformly for $h \in] 0,1], t \geqq T_{0}$

ii) if $a(h) \rightarrow \infty$ when $h \rightarrow 0$, then:

$$
\left\|r_{\alpha}(t, h)\right\|=O\left(a(h)^{-1} e^{-a t(1-\alpha)}\right)
$$

uniformly for $h \in] 0,1], t \geqq T_{0}$.

iii) $\left\|\chi(|x| \leqq v \alpha t) r_{\text {out }, \alpha}(t, h)\right\|=\frac{O\left(h^{\infty}\right)}{\langle t\rangle^{\infty}}$ uniformly for $\left.\left.h \in\right] 0,1\right], t \geqq T_{0}$.

Proof of Theorem 3.7. we have:

$$
\begin{aligned}
& e^{-i t\left(P_{h}-E(h)\right) / h} \chi\left(x, h D_{x}\right) u_{h} \\
& =\chi\left(x, h D_{x}\right) u_{h}-i h^{-1} \int_{0}^{t} e^{-i s\left(P_{h}-E(h)\right) / h}\left(P_{h}-E(h)\right) \chi\left(x, h D_{x}\right) u_{h} d s \\
& =\chi\left(x, h D_{x}\right) u_{h}-i h^{-1} \int_{0}^{t} e^{-i s\left(P_{h}-E(h)\right) / h} \chi\left(x, h D_{x}\right)\left(P_{h}-E(h)\right) u_{h} d s \\
& \quad-i h^{-1} \int_{0}^{t} e^{-i s\left(P_{h}-E(h)\right) / h}\left[P_{h}, \chi\right] u_{h} d s .
\end{aligned}
$$

From Definition 3.4, it follows that $\chi\left(x, h D_{x}\right)\left(P_{h}-E(h)\right) u_{h}=r_{\infty, 1}(h)$ has a norm $O\left(h^{\infty}\right)$.

Also $i h^{-1}\left[P_{h}, \chi\left(x, h D_{x}\right)\right]$ is a p.d.o. supported in $\left\{|x| \geqq R_{1}\right\}$, (see (3.1)) so

$$
i h^{-1}\left[P_{h}, \chi\left(x, h D_{x}\right)\right] u_{h}=r_{\infty, 2}(h)+\chi_{\text {out }}\left(x, h D_{x}, h\right) u_{h},
$$

where $r_{\infty, 2}$ has an $L^{2}$ norm $O\left(h^{\infty}\right)$ and $\chi_{\text {out }}(x, \xi, h)$ is supported in an outgoing region $\left\{(x, \xi)\left|G(x, \xi) \geqq C_{1},\right| x \mid \geqq R_{1}\right\}$ by Definition 3.4 iv). On the support of $\chi_{\text {out }}, u_{h}$ satisfies $\left(-h^{2} \Delta+V_{r}(x)+W(x)-E(h)\right) u_{h}=O\left(h^{\infty}\right)$, since $W$ and $V_{s}$ vanish in $\left\{|x| \geqq R_{1}\right\}$. Using then the functional calculus of Helffer-Robert [He-Ro], generalized to non-conformal metrics in [Ge1], we easily get that:

$$
\chi\left(P_{h, 1}\right) \chi_{\text {out }} u_{h}=\chi_{\text {out }} u_{h}+O_{L^{2}}\left(h^{\infty}\right)
$$

where $\chi(\lambda)$ is a cutoff function supported in $I$. We also have by combining Proposition 5.4 and Theorem 7.3:

$$
\begin{aligned}
e^{-i s P_{h} / h} \chi_{\text {out }} u_{h} & =e^{-i s P_{h} / h} \chi\left(P_{h}\right) \chi_{\text {out }} u_{h}+O\left(h^{\infty}\right) \\
& =e^{-i s P_{h, 1} / h} \chi\left(P_{h, 1}\right) \chi_{\text {out }} u_{h}+O\left(h^{\infty}\right) .
\end{aligned}
$$


Here $\chi(\lambda)$ is a cutoff function supported in $I$ and equal to 1 near $\lambda_{0}$. Finally we get:

$$
\begin{aligned}
e^{-i t P_{h} / h} \chi\left(x, h D_{x}\right) u_{h}= & e^{-i t E(h) / h} \chi\left(x, h D_{x}\right) u_{h} \\
& -\int_{0}^{t} e^{-i(t-s) E(h) / h} e^{-i s P_{h, 1 / h}} \chi\left(P_{h, 1}\right) \chi_{\text {out }} u_{h} d s \\
& +\int_{0}^{t} e^{-i(t-s) E(h) / h} r_{\infty}(s) d s,
\end{aligned}
$$

where $\left\|r_{\infty}(s)\right\|=O\left(h^{\infty}\right)$ uniformly in $s$.

The integral of the last term is bounded by:

$$
O\left(h^{\infty}\right) a^{-1}\left(1-e^{-a t}\right)
$$

uniformly in $t$, which proves $i$ ).

To prove the Theorem, we just have to establish the desired estimates on:

$$
r_{\text {out }}(t)=\int_{0}^{t} e^{-i(t-s) E(h) / h} e^{-i s P_{h, 1} / h} \chi\left(P_{h, 1}\right) \chi_{\text {out }} u_{h} d s .
$$

If $\chi_{0}(x)$ is as in the statement of the Theorem 3.7, we have:

$$
\left\|\chi_{0}(x) e^{-i s P_{h, 1 / h}} \chi\left(P_{h, 1}\right) \chi_{\text {out }}\right\|=\frac{O\left(h^{\infty}\right)}{\langle s\rangle^{\infty}} .
$$

This follows from Theorem 7.2 and from Egorov's Theorem for small $s$. This gives:

$$
\left\|\chi_{0}(x) r_{\text {out }}(t)\right\| \leqq \int_{0}^{t} e^{-(t-s)|\operatorname{Im} E(h)| / h} C_{N} \frac{h^{N}}{\langle s\rangle^{N}} d s
$$

for any $N \in \mathbb{N}$. Then it suffices to apply Proposition B.1 in Appendix 2 to prove ii), which completes the proof of the theorem.

Proof of Theorem 3.8. We write $r_{\text {out }}(t)=r_{\alpha}(t)+r_{\text {out }, \alpha}(t)$ where:

$$
\begin{aligned}
r_{\alpha}(t) & =\int_{0}^{\alpha t} e^{-i(t-s) E(h) / h} e^{-i s P_{h, 1 / h}} \chi\left(P_{h, 1}\right) \chi_{\text {out }} u_{h} d s, \\
r_{\text {out }, \alpha}(t) & =\int_{\alpha t}^{t} e^{-i(t-s) E(h) / h} e^{-i s P_{h, 1 / h}} \chi\left(P_{h, 1}\right) \chi_{\text {out }} u_{h} d s .
\end{aligned}
$$

Let us first estimate $\left\|r_{\alpha}(t)\right\|$ : the computation is rejected to the Appendix. By Proposition B.2, we get:

- if $a(h)=O_{h}(1)$ :

$$
\left\|r_{\alpha}(t)\right\| \leqq C e^{-a t(1-\alpha)}\left(1+(a)^{-1 / 2}\left(1-e^{-C a t}\right)^{1 / 2}\right)
$$

- if $a(h) \rightarrow \infty$ when $h \rightarrow 0$ :

$$
\left\|r_{\alpha}(t)\right\| \leqq C a^{-1} e^{-a t(1-\alpha)} .
$$

Let us now consider $r_{\text {out }, \alpha}(t)$ : by Theorem 7.2 we get that:

$$
\left\|\chi(|x| \leqq v \alpha t) e^{-i s P_{h, 1} / h} \chi\left(P_{h, 1}\right) \chi_{\text {out }} u_{h}\right\| \leqq C_{N} h^{N}\langle s\rangle^{-N},
$$

uniformly for $s \geqq \alpha t$. 
Then we get immediately that:

$$
\left\|\chi(|x| \leqq v \alpha t) r_{\text {out }, \alpha}(t)\right\|=\frac{O\left(h^{\infty}\right)}{\langle t\rangle^{\infty}}
$$

which completes the proof of Theorem 3.8.

3.D. Propagation Estimates Near a Non-Trapping Energy Level. We consider in this subsection an Hamiltonian.

$$
P_{h}=-h^{2} \Delta+V(x)
$$

satisfying the conditions of Sect. 2 with $V_{s}=0$, and an energy interval $I$ such that $I$ is non-trapping for $p(x, \xi)=\xi^{2}+V(x)$. We denote by $\chi_{1}(\lambda) \in C_{0}^{\infty}(\mathbb{R})$ a cutoff function supported in $I$ so that Corollary 6.5 and Theorem 7.2 hold with the energy cutoff $\chi_{1}\left(P_{h}\right)$. Then we have the following result:

Theorem 3.9. Let $\chi_{0}(x, \xi) \in C_{0}^{\infty}\left(T^{*} \mathbb{R}^{n}\right)$ be a cutoff function. Then there exist $\delta>0$ and a time $T_{0}=T\left(\chi_{0}\right)$ such that:

$$
\text { for } t \geqq T_{0}\left\|\chi(|x| \leqq \delta t) e^{-i t P_{h} / h} \chi_{1}\left(P_{h}\right) \chi_{0}\left(x, h D_{x}\right)\right\|=O\left(\frac{h^{\infty}}{\langle t\rangle^{\infty}}\right) .
$$

The important part in this estimate is of course the factor $O\left(h^{\infty}\right)$.

Proof. The proof will be an easy application of Theorem 7.2 and Egorov's theorem (see [Ro]).

We first take another cutoff function $\chi_{2}(\lambda)$ with $\chi_{1}(\lambda) \chi_{2}(\lambda)=\chi_{1}(\lambda)$ and $\chi_{2}$ is supported in $I$. Then using the functional calculus (see [He-Ro], [Ge1]) we get that:

$$
\chi_{2}\left(P_{h}\right)=\chi_{p s}\left(x, h D_{x}, h\right)+R_{\infty}(h)
$$

where $\chi_{p s}\left(x, h D_{x}, h\right)$ is a pseudodifferential operator with total symbol supported in $p^{-1}(I)$, and $R_{\infty}(h)$ has a norm $O\left(h^{\infty}\right)$ between any weighted Sobolev spaces $H_{\langle x\rangle^{k}}^{s}$. So

$$
\chi_{1}\left(P_{h}\right) \chi_{0}\left(x, h D_{x}\right)=\chi_{1}\left(P_{h}\right) \chi_{p s}\left(x, h D_{x}, h\right) \chi_{0}\left(x, h, D_{x}\right)+R_{\infty}(h) \chi_{0}\left(x, h D_{x}\right) .
$$

Since $\chi_{\text {out }, 1}=R_{\infty}(h) \chi_{0}\left(x, h D_{x}\right)$ satisfies the estimates iii) of Proposition 6.4, we have:

$\left\|\chi(|x| \leqq v(t+1)) e^{-i t P_{h} / h} \chi_{\text {out }, 1}\right\|=\left\|\chi(|x| \leqq v(t+1)) e^{-i t P_{h} / h} \chi_{2}\left(P_{h}\right) \chi_{\text {out }, 1}\right\|=O\left(\frac{h^{\infty}}{\langle t\rangle^{\infty}}\right)$

by Theorem 7.2 .

We consider now $\chi_{p s}\left(x, h D_{x}, h\right) \chi_{0}\left(x, h D_{x}\right)$ which is a pseudodifferential operator supported in $p^{-1}(I)$. By Egorov's theorem (see [Ro]) we get:

$$
e^{-i t P_{h} / h} \chi_{p s}\left(x, h D_{x}, h\right) \chi_{0}\left(x, h D_{x}\right)=\chi_{t}\left(x, h D_{x}, h\right) e^{i t P_{h} / h},
$$

where $\chi_{t}\left(x, h D_{x}, h\right)$ is a p.d.o. with essential support in

$$
\exp t H_{p}\left(\operatorname{supp} \chi_{p s}(x, \xi, h) \chi_{0}(x, \xi)\right) \text {. }
$$

Since $I$ is non-trapping, we can choose $T=T\left(\chi_{0}\right)$ such that $\chi_{T}\left(x, h D_{x}, h\right)$ has essential support in a region $\left\{(x, \xi) \mid G(x, \xi) \geqq C_{1}\right\}$, where $C_{1} \gg 1$, and $G$ is the escape 
function modified as in Proposition 2.2 Hence we can apply Theorem 7.2 with $\chi_{\text {out }}=\chi_{T}\left(x, h D_{x}, h\right)$ for $t \geqq T$, which proves Theorem 3.9.

\section{Examples}

In this section, we will give examples of quasiresonance states and of quasiresonances. We first show that for dilation analytic two-body potentials, exact resonance states are quasiresonance states in the sense of Definition 3.4. We will then construct quasiresonances states and quasiresonances in situations where one has shown the existence of true resonances, at least for two-body dilation analytic potentials. Namely we will study the cases when the set of trapped trajectories in $p^{-1}\left(\lambda_{0}\right), K \cap p^{-1}\left(\lambda_{0}\right)$ is either a closed hyperbolic trajectory (see [Ge-Sj]) or an unstable equilibrium point. This last case occurs for example when $V_{r}$ has a non-degenerate maximum (see $[\mathrm{Sj}],[\mathrm{B}-\mathrm{C}-\mathrm{D}])$. Although we make these constructions for smooth potentials, it is clear that one can admit a compactly supported singular potential, as long as the frequency set of the quasiresonances we construct does not intersect with its singular support. Finally we will treat under stronger hypotheses the case of the shape resonances, where the methods of Sect. 3 no longer apply.

4.A. Dilation Analytic Potentials. We assume now that $P_{h}$ is a 2-body Hamiltonian and that $V_{r}(x)$ is dilation analytic in a sector $\left\{x \in \mathbb{C}^{n}|| \operatorname{Im} x \mid \leqq \varepsilon\langle\operatorname{Re} x\rangle\right\}$. Then one can define resonances near $I$ with $I \subset \mathbb{R}_{+}^{*}$ by the method of complex canonical transformations developped in successive degrees of generality by Aguilar-Combes [Ag-Co], Balslev-Combes [Ba-Co], Sigal [S1] Hunziker [Hu1] (see CombesDuclos-Klein-Seiler [C-D-K-S], Briet-Combes-Duclos [B-C-D], HislopSigal [Hi-Si], Sigal [2] for semiclassical versions) and Helffer-Sjöstrand [He-Sj2].

In this last approach, resonant functions are distributions in some Hilbert spaces denoted by $H\left(\Lambda_{t G}, m\right)$ of Sobolev type with microlocal exponential weights. Then it is very easy to see, using for example the characterization of frequency set through F.B.I. transforms (see Martinez [Ma]) that a resonant function is also a quasiresonance state since it has no frequency set in $\left\{(x, \xi) \mid G(x, \xi) \leqq-C_{1}\right\}$ for $C_{1}>1$ (see also Remark 2).

4.B. Quasiresonances Generated by a Closed Hyperbolic Trajectory. This situation was studied by Gérard-Sjöstrand [Ge-Sj] for dilation analytic potentials. We put ourselves under the hypotheses of Sect. 2 and fix an energy level $\lambda_{0}$. The energy interval we consider will be of the form $I=\left[\lambda_{0}-\varepsilon, \lambda_{0}+\varepsilon\right]$ for $\varepsilon$ small enough. We assume that the set of trapped trajectories in $p^{-1}\left(\lambda_{0}\right)$ is a closed trajectory $\gamma^{0}$ of hyperbolic type, i.e. the eigenvalues of the linearized Poincaré map are not of modulus 1 .

We first fix some notations. For $\lambda$ close to $\lambda_{0}$ we denote by $\gamma^{\lambda}$ the (unique) closed trajectory of $H_{p}$ in $p^{-1}(\lambda) . \gamma^{\lambda}$ is still hyperbolic for $\lambda$ near $\lambda_{0}$ and we denote by $\theta_{1}(\lambda), \ldots, \theta_{n-1}(\lambda)$ the eigenvalues of modulus $>1$ of the Poincaré map $P^{\lambda}$ of $\gamma^{\lambda}$.

To completely specify $P^{\lambda}$, we fix in $p^{-1}(\lambda)$ a symplectic manifold $\Pi^{\lambda}$ of codimension 2 in $T^{*} \mathbb{R}^{n}$ which is transverse to $H_{p}$ at $\gamma^{\lambda} \cap \Pi^{\lambda}=\rho^{\lambda}$. Then we take $P^{\lambda}$ to be the first return map in $\Pi^{\lambda}$ and $P^{\lambda}$ is a symplectic map for the symplectic structure on $\Pi^{\lambda}$. We denote by $\Lambda_{+(-)}^{\lambda}$ the stable outgoing (incoming) Lagrangian 
manifold of $\gamma^{\lambda} . \Lambda_{+}^{\lambda}$ is tangent at $\rho^{\lambda}$ to the space generated by $H_{p}$ and the eigenvectors of $D_{\rho^{\lambda}} P^{\lambda}$ with eigenvalues $\theta_{i}(\lambda) 1 \leqq i \leqq n-1$.

We denote by $\Gamma_{ \pm}^{\lambda}$ the manifold $\Lambda_{ \pm}^{\lambda} \cap \Pi^{\lambda}$, which is still Lagrangian in $\Pi^{\lambda}$ and invariant under $P^{\lambda}$.

For $\alpha \in \mathbb{N}^{n-1}$, we denote by $N(\alpha)$ the number of $\beta \in \mathbb{N}^{n-1}$ such that $\theta(\lambda)^{-\beta}=\theta(\lambda)^{-\alpha}$. We denote by $\sigma$ the Maslov index of $\gamma^{0}$, by $C(\lambda)=\int \xi d x$ the action along $\gamma^{\lambda}$, and by $T(\lambda)=C^{\prime}(\lambda)$ the period of $\gamma^{\lambda}$.

Then we have:

Proposition 4.1. For any $\alpha \in \mathbb{N}^{n-1}$, there exist aN $(\alpha) \times N(\alpha)$ matrix

$$
M(\lambda, z, h) \cong \sum_{0}^{\infty} M_{j}(\lambda, z) h^{j}
$$
where $M_{0}(\lambda, z)$ has the eigenvalue $\theta(\lambda)^{-\alpha} \prod_{1}^{n-1}\left|\theta_{i}(\lambda)\right|^{-1 / 2} e^{i T(\lambda) z}$ with multiplicity $N(\alpha)$
such that:

i) $\lambda+h z$ is a quasiresonance if

$$
\operatorname{det}\left(e^{i C(\lambda) / h} e^{i \pi \sigma / 4} M(\lambda, z, h)-1\right)=0,
$$

ii) the quasiresonant state $u_{h}$ associated with $E_{\alpha}(h)=\lambda+h z$ is a Lagrangian distribution living on $\Lambda_{+}^{\lambda}$.

Remark 4. As in $[\mathrm{Ge}-\mathrm{Sj}]$ one shows easily that:

$$
\begin{aligned}
& \operatorname{Im} E_{\alpha}(h)=\left(\log \left(\prod_{1}^{n-1}\left|\theta_{i}(\lambda)\right|^{-1 / 2}\right)-\sum_{1}^{n-1} \alpha_{i} \log \theta_{i}(\lambda)\right) T(\lambda)^{-1} h+o(h) \\
& \operatorname{Re} E_{\alpha}(h)=\lambda+T(\lambda)^{-1} h\left(\sigma \frac{\pi}{4}+2 k \pi-C(\lambda) / h\right)+o(h),
\end{aligned}
$$

where $k \in \mathbb{Z}$ is such that $2 k \pi-C(\lambda) / h=O(1)$.

Proof. The construction of quasiresonant states will be separated in three steps:

Step 1. We find quantization conditions to have a solution $u_{h}$ of:

$$
P_{h} u_{h}-(\lambda+z h) u_{h}=O\left(h^{\infty}\right)
$$

microlocally near $\gamma^{0}$. Here $z=O_{h}(1)$ and $P_{h}$ could be any pseudodifferential operator $p\left(x, h D_{x}, h\right)$. Instead of directly constructing $u_{h}$ as a Lagrangian distribution on $\Lambda_{+}^{\lambda}$, we reduce (4.1) to construction of solutions of

$$
K_{\lambda}(z) v_{h}=v_{h}+O\left(h^{\infty}\right)
$$

microlocally near $\rho^{\lambda}$, where $v_{h}$ lives on $\Pi^{\lambda}$ and $K_{\lambda}(z)$ is the "quantized Poincaré map."

We first choose a canonical transformation $\chi_{\lambda}$ which sends $\rho^{\lambda}$ to $(0,0), \Pi^{\lambda}$ on $\left\{x_{1}=\xi_{1}=0\right\}, \Gamma_{+}^{\lambda}$ on $\left\{\xi^{\prime}=0\right\}, \Gamma_{-}^{\lambda}$ on $\left\{x^{\prime}=0\right\}$, where $x=\left(x_{1}, x^{\prime}\right), \xi=\left(\xi_{1}, \xi^{\prime}\right)$ (see [Ge-Sj, Sect. 2]). Since $H_{p}$ is transversal to $\Pi^{\lambda}$, we can also assume (possibly after exchanging $x_{1}$ and $\left.\xi_{1}\right)$ that $\frac{\partial p}{\partial \xi_{1}}(0,0) \neq 0$ in the new coordinates. $\chi_{\lambda}$ is defined near 
$\rho^{\lambda}$ and we extend it arbitrarily in a large region of $T^{*} \mathbb{R}^{n}$. We quantize $\chi_{\lambda}$ by a Fourier integral operator $T_{\lambda}$ and consider everything after conjugation by $T_{\lambda}$.

In these new symplectic coordinates we can construct an operator $M_{\lambda}(z)$ such that if $v_{h}$ is defined microlocally near $(0,0)$ in $\Pi^{\lambda}, u_{h}=M_{\lambda}(z) v_{h}$ solves:

$$
\left\{\begin{array}{c}
\left(P_{h, \lambda}-E_{\lambda}\right) u_{h}=O\left(h^{\infty}\right) \text { microlocally near } \gamma^{\lambda} \\
\left.u_{h}\right|_{x_{1}=0}=v_{h}
\end{array} .\right.
$$

Here $P_{h, \lambda}=T_{\lambda} P_{h} T_{\lambda}^{-1}$, and $E_{\lambda}=\lambda+z h$. We will see below that the existence of $u_{h}$ solution to (4.1) is equivalent to the existence of a solution to (4.3) which is univalued under the operation of "moving one loop around $\gamma^{\lambda}$."

$M_{\lambda}(z) v_{h}$ can be constructed near $x_{1}=0$ as a Fourier integral operator:

$$
M_{\lambda, 1}(z) v_{h}=(2 \pi h)^{-n+1} \int e^{i\left(S_{\lambda}\left(x_{1}, x^{\prime}, \eta^{\prime}\right)-y^{\prime} \cdot \eta^{\prime}\right) / h} a_{\lambda}\left(x, \eta^{\prime}, z, h\right) v_{h}\left(y^{\prime}\right) d y^{\prime} d \eta^{\prime},
$$

where $S_{\lambda}$ solves the Hamilton-Jacobi equation:

$$
\left\{\begin{array}{l}
p_{\lambda}\left(x, \frac{\partial S_{\lambda}}{\partial x}\right)=\lambda \\
\left.S_{\lambda}\right|_{x_{1}=0}=x^{\prime} \cdot \eta^{\prime}
\end{array},\right.
$$

where $p_{\lambda}$ is the symbol of $P_{h, \lambda}$, and:

$$
a_{\lambda}\left(x, \eta^{\prime}, z, h\right)=\sum_{0}^{\infty} a_{\lambda, j}\left(x, \eta^{\prime}, z\right) h^{j}
$$

with $a_{\lambda, j}$ solving the transport equations.

Equation (4.4) can be solved near $x_{1}=0$ since $H_{p}$ is transversal to $\Pi^{\lambda}$ at $\rho^{\lambda}$. The global construction of $M_{\lambda}(z) v_{h}$ as a Lagrangian distribution amounts to construction of the Lagrangian manifold $\Lambda_{\lambda, \eta^{\prime}}$ which is the image of $\left\{\xi^{\prime}=\eta^{\prime}\right\}$ under the $H_{p}$ flow in $p^{-1}(\lambda)$.

Let us write the global construction of $M_{\lambda}(z) v_{h}$ as:

$$
M_{\lambda}(z) v_{h}=\sum_{1}^{N} M_{\lambda, i}(z) v_{h},
$$

where $M_{\lambda, i}(z) v_{h}$ is a Lagrangian distribution solution to (4.3) near some point $\rho_{\lambda, i}$ on $\gamma^{\lambda}$, and $M_{\lambda, N}(z) v_{h}$ lives near $\rho^{\lambda}$.

The $M_{\lambda, i}(z) v_{h}$ patch together as a global solution to (4.3) except for $M_{\lambda, N}(z) v_{h}$ and $M_{\lambda, 1}(z) v_{h}$. The quantization condition for the existence of a solution to (4.1) is that $M_{\lambda, N}(z) v_{h}=M_{\lambda, 1}(z) v_{h}+O\left(h^{\infty}\right)$. Since $M_{\lambda, N}(z) v_{h}$ and $M_{\lambda, 1}(z) v_{h}$ solve (4.1) locally near $\rho^{\lambda}$, they coincide (modulo $O\left(h^{\infty}\right)$ ) if and only if:

$$
\left.M_{\lambda, N}(z) v_{h}\right|_{x_{1}=0}=v_{h}+O\left(h^{\infty}\right) \text { near }(0,0) .
$$

We will denote by $K_{\lambda}(z)$ the operator:

$$
\left.v_{h} \mapsto M_{\lambda, N}(z) v_{h}\right|_{x_{1}=0}
$$

acting on $D^{\prime}\left(\mathbb{R}^{n-1}\right)$ and defined microlocally near $(0,0)$.

Let us now describe $K_{\lambda}(z)$ in more details. $K_{\lambda}(z)$ is a F.I.O. associated with the canonical transformation $P^{\lambda}$. Since $D_{\rho^{\lambda}} P^{\lambda}$ is of the form $\left(\begin{array}{cc}A_{\lambda} & 0 \\ 0 & \left({ }^{t} A_{\lambda}\right)^{-1}\end{array}\right)$ in the new 
coordinates, where $A_{\lambda}$ is a matrix with eigenvalues $\theta_{i}(\lambda), K_{\lambda}(z)$ can be written as:

$$
K_{\lambda} v_{h}=(2 \pi h)^{-n+1} \int e^{i\left(\varphi_{\lambda}\left(x^{\prime}, \eta^{\prime}\right)-y^{\prime} \cdot \eta^{\prime}\right) / h} k_{\lambda}\left(x^{\prime}, \eta^{\prime}, z, h\right) v_{h}\left(y^{\prime}\right) d y^{\prime} d \eta^{\prime},
$$

where

$$
\begin{gathered}
\varphi_{\lambda}\left(x^{\prime}, \eta^{\prime}\right)=\varphi_{\lambda}(0,0)+A_{\lambda}^{-1} x^{\prime} \cdot \eta^{\prime}+O\left(\left|\left(x^{\prime}, \eta^{\prime}\right)\right|^{3}\right), \\
k_{\lambda}\left(x^{\prime}, \eta^{\prime}, z, h\right) \cong \sum_{0}^{\infty} k_{\lambda, j}\left(x^{\prime}, \eta^{\prime}, z\right) h^{j} .
\end{gathered}
$$

We will now compute $\varphi_{\lambda}(0,0)$ and $k_{\lambda, 0}(0,0, z)$.

By Hamilton-Jacobi theory we know that:

$$
\varphi_{\lambda}(0,0)=C(\lambda)=\int_{\gamma^{\lambda}} \xi d x
$$

Note that $C^{\prime}(\lambda)=T_{\lambda}$, where $T_{\lambda}$ is the period of $\gamma^{\lambda}$.

To compute $k_{\lambda, 0}(0,0, z)$, we have to take into account the transport equation and the Maslov bundle on $\Lambda_{\lambda, 0}$. If we consider again $M_{\lambda, 1}$ near $x_{1}=0$ (fixing $\eta^{\prime}=0$ ) and compute the principal symbol $m_{\lambda}$ of $M_{\lambda, 1}$ as a half density, we get:

$$
m_{\lambda}=a_{\lambda}(x, 0, z)|d x|^{1 / 2} \quad \text { with } a_{\lambda}(0,0, z)=1,
$$

since $\Lambda_{\lambda, 0}$ can be parametrized by $x$ near $x_{1}=0$. It is well known (see [Hö, Theorem 25.2.4]) that the principal symbol $m_{\lambda}$ of $M_{\lambda}$ satisfies:

$$
\mathscr{L}_{\boldsymbol{H}_{\boldsymbol{p}}} m_{\lambda}-i z m_{\lambda}=0
$$

(remember that the subprincipal symbol of a Schrödinger operator vanishes). Here $\mathscr{L}_{H_{p}} m_{\lambda}$ denotes the Lie derivative of $m_{\lambda}$.

So if we denote by $\Phi^{t}$ the flow of $H_{p}$ on $\Lambda_{\lambda, 0}$, one has:

$$
\left(\Phi^{T_{\lambda}}\right)^{*} m_{\lambda, N}=m_{\lambda, 1} \times e^{i T_{\lambda z}},
$$

where $m_{\lambda, j}$ is the principal symbol of $M_{\lambda, j}$.

At $(0,0), m_{\lambda, N}$ is equal to $k_{\lambda, 0}(0,0, z)|d x|^{1 / 2}$ (modulo the Maslov factor).

According to [Ge-Sj, Sect. 2] the differential of $\Phi^{T_{\lambda}}$ restricted to $\Lambda_{\lambda, 0}=\Lambda_{\lambda,+}$ is of the form $\left(\begin{array}{cc}1 & 0 \\ 0 & A_{\lambda}\end{array}\right)$ so:

$$
k_{\lambda}(0,0, z)\left|\operatorname{det} A_{\lambda}\right|^{1 / 2}|d x|^{1 / 2}=e^{+i T_{\lambda} z}|d x|^{1 / 2} .
$$

modulo the Maslov factor.

If we take into account this factor we finally get:

$$
k_{\lambda}(0,0, z)=e^{i T_{\lambda z}}\left|\operatorname{det} A_{\lambda}\right|^{-1 / 2} e^{i \pi \sigma / 4}
$$

where $\sigma$ is the Maslov index of $\gamma^{0}$. We will denote $\left|\operatorname{det} A_{\lambda}\right|^{-1 / 2}$ by $b_{\lambda}$. This completes Step 1.

Step 2. We will describe the quantization conditions for (4.5). A similar problem (with no dependence on $\lambda$ ) has been treated in [Ge2], so we will be quite sketchy.

If we replace $K_{\lambda}(z)$ by its linearization $\tilde{K}_{\lambda}(z)$ at $(0,0)$, i.e. we replace $\phi_{\lambda}$ by $C(\lambda)+A_{\lambda}^{-1} x^{\prime} \cdot \eta^{\prime}$, and $k_{\lambda, 0}\left(x^{\prime}, \eta^{\prime}, z, h\right)$ by $k_{\lambda, 0}(0,0, z)$ we see that:

$$
\tilde{K}_{\lambda}(z) v_{h}=e^{i C(\lambda) / h}\left|\operatorname{det} A_{\lambda}\right|^{-1 / 2} e^{i T_{\lambda} z+i \pi \sigma / 4} v_{h}\left(A_{\lambda}^{-1} x^{\prime}\right) .
$$


The eigenvectors of $v_{h} \mapsto v_{h}\left(A_{\lambda}^{-1} x^{\prime}\right)$ are polynomials with eigenvalues

$$
\theta(\lambda)^{-\alpha}=\theta_{1}(\lambda)^{-\alpha_{1}} \cdots \theta_{n-1}(\lambda)^{-\alpha_{n}-1}
$$

for $\alpha \in \mathbb{N}^{n-1}$. So the quantization condition corresponding to $\tilde{K}_{\lambda}(z) v=v$ is:

$$
C(\lambda)=(2 k \pi-\sigma / 4 \pi) h+i \log b_{\lambda} h-T(\lambda) z h-i h \sum_{1}^{n-1} \alpha_{i} \log \theta_{i}(\lambda)
$$

for $k \in \mathbb{Z}$. This can be inverted since $T(\lambda) \neq 0$. The quantization conditions for $K_{\lambda}(z)$ can now be found by perturbation following the arguments of [Ge2]. One gets the quantization conditions given in Proposition 4.1. One also gets that $v_{h}$ solution of (4.2) is a Lagrangian distribution living on $\Gamma_{+}^{\lambda}$.

Step 3. We extend globally the solutions of (4.1). If $v_{h}$ is the solution of (4.2), $u_{h}=M_{\lambda}(z(h)) v_{h}$ is a Lagrangian distribution living on $\Lambda_{+}^{\lambda}$, which satisfies $P_{h} u_{h}-(\lambda+z(h)) u_{h}=O\left(h^{\infty}\right)$ near $\gamma^{0}$.

Since $\gamma^{\lambda}$ is the only trapped trajectory on $p^{-1}(\lambda)$, we can extend $u_{h}$ to any open neighborhood of $\Lambda_{+}^{\lambda}$ in such a way that it still solves $P_{h} u_{h}-(\lambda+z(h)) u_{h}=O\left(h^{\infty}\right)$ in this neighborhood. To solve Schrödinger equation everywhere, it suffices to replace $u_{h}$ by $\chi\left(P_{h}\right) u_{h}$, where $\chi(\lambda)$ is supported near $\lambda_{0}$, and to use the functional calculus of [He-Ro]. This completes the proof of Proposition 4.1.

4.C. Quasiresonances Generated by a Potential Maximum. This situation was studied by $\mathrm{Sjöstrand}[\mathrm{Sj}]$, Briet-Combes-Duclos [B-C-D] for dilation analytic potentials. As in subsection 4.B, we take $I=\left[\lambda_{0}-\varepsilon, \lambda_{0}+\varepsilon\right]$, for $\lambda_{0} \in \mathbb{R}$ and $\varepsilon$ small enough and we assume that $V$ satisfies the conditions of Sect. 2 .

We assume that the set of trapped trajectories in $p^{-1}\left(\lambda_{0}\right)$ is a point $\left(x_{0}, 0\right)$ where $V\left(x_{0}\right)=\lambda_{0}, \nabla_{x_{0}} V=0, V^{\prime}\left(x_{0}\right)$ is negative definite. If the eigenvalues of $V^{\prime}$ at $x_{0}$ are denoted by $\lambda_{j}, 1 \leqq j \leqq n$, the eigenvalues of the linearized matrix $F_{p}$ of $H_{p}$ at $\left(x_{0}, 0\right)$ are the $\pm 2\left(-\lambda_{j}\right)^{1 / 2}= \pm \mu_{j}, 1 \leqq j \leqq n$.

For $\alpha \in \mathbb{N}^{n}$ we denote by $N(\alpha)$ the number of $\beta \in \mathbb{N}^{n}$ with $\alpha \cdot \mu=\beta \cdot \mu$, where $\mu=\left(\mu_{1}, \ldots, \mu_{n}\right)$. We denote by $\Lambda_{+}$the stable outgoing manifold of $\left(x_{0}, 0\right)$ (see $[\mathrm{A}-\mathrm{M}])$. Then we have:

Proposition 4.2. For $\alpha \in \mathbb{N}^{n}$ there exist a $N(\alpha) \times N(\alpha)$ matrix $F(z, h) \cong \sum_{0}^{\infty} F_{j}(z) h^{j}$, where $F_{0}(z)$ has the eigenvalue $-i \sum_{1}^{n}\left(\alpha_{j}+\frac{1}{2}\right) \mu_{j}-z$ with multiplicity $N(\alpha)$, such that:

i) $\lambda_{0}+z h$ is a quasiresonance if: $\operatorname{det}(F(z, h))=0$

ii) the quasiresonant state $u_{h}$ associated to $E_{\alpha}(h)=\lambda_{0}+z h$ is a Lagrangian distribution living on $\Lambda_{+}$.

Proof. By the stable manifold theorem (see [A-M]) there exist two Lagrangian manifolds $\Lambda_{ \pm}$tangent at $\left(x_{0}, 0\right)$ to the span of eigenspaces of $F_{p}$ with positive (respectively negative) eigenvalues, which are $H_{p}$ invariant. By a change of symplectic coordinates we can locally send $\left(x_{0}, 0\right)$ to $(0,0), \Lambda_{+}$to $\{\xi=0\}$, and $\Lambda_{-}$to $\{x=0\}$. If $T$ is a F.I.O. associated with this change of coordinates, and $\widetilde{P}=T P_{h} T^{-1}$, we have:

$$
\tilde{P}=\tilde{p}\left(x, h D_{x}\right)+h \tilde{p}_{-1}\left(x, h D_{x}\right)+O\left(h^{2}\right)
$$


with $\tilde{p}(x, \xi)=\langle B(x, \xi) x, \xi\rangle$, where $B(0,0)$ has the eigenvalues $\mu_{1}, \ldots, \mu_{n}$. We will use also the subprincipal symbol of $P$, defined as:

$$
p_{\text {sub }}(x, \xi)=p_{-1}(x, \xi)+\frac{i}{2} \sum \partial_{x_{j}} \partial_{\xi} p(x, \xi)
$$

Since at double characteristic points the subprincipal symbol is invariant under conjugation by Fourier integral operators, we see that $\tilde{p}_{-1}(0,0)=-\frac{i}{2} \sum_{1}^{n} \mu_{j}$.

We can write:

$$
\tilde{P}=\sum_{|\alpha|,|\beta|=1} x^{\alpha} a_{\alpha, \beta}\left(x, h D_{x}\right)\left(h D_{x}\right)^{\beta}+h a_{-1}\left(x, h D_{x}\right)+h^{2} a_{-2}+\cdots .
$$

To compute $a_{-1}(0,0)$ we still use the invariance of the subprincipal symbol and get:

so $a_{-1}=-\frac{i}{2} \sum_{1}^{n} \mu_{j}$

$$
a_{-1}(0,0)+\frac{i}{2} \sum_{j=1}^{n} \partial_{x_{j}} \partial_{\xi_{j}} \tilde{p}(0,0)=\sigma_{\text {sub }}(\tilde{P})(0,0)=0
$$

Now as in Helffer-Sjöstrand ([He-Sj1] Proof of Theorem 3.7) we introduce the linearized operator:

$$
\tilde{P}_{0}=\sum_{|\alpha|=|\beta|=1} x^{\alpha} a_{\alpha, \beta}(0,0)\left(h D_{x}\right)^{\beta}+h a_{-1}(0,0)
$$

and the space $S_{1 / 2}^{m, k}$ of formal symbols of the form:

$$
u(x, h)=\sum_{0}^{\infty} u_{j}(x) h^{-m+j}, \quad j \in \mathbb{N} / 2,
$$

where $u_{j}=O\left(|x|^{(k-2 j)+}\right)$.

$h^{-1} \widetilde{P}_{0}$ sends $S_{1 / 2}^{m, k}$ into itself and $h^{-1}\left(\tilde{P}-\tilde{P}_{0}\right)$ sends $S_{1 / 2}^{m, k}$ into $S_{1 / 2}^{m, k+1}$.

Now the eigenvalues of $h^{-1} \tilde{P}_{0}$ are the $-i \sum_{1}^{n}\left(\alpha_{j}+\frac{1}{2}\right) \mu_{j}, \alpha \in \mathbb{N}^{n}$ with eigen-
ctors $x^{\alpha}$.

The quantization condition can now be obtained exactly as in [He-Sj1, Theorem 3.7] or [He, Chap. 2]. It is also clear from the proof that the quasiresonant states are Lagrangian distributions living on $\Lambda_{+}$. One can extend the quasiresonant states to any neighborhood of $\Lambda_{+}$since $\left(x_{0}, 0\right)$ is the only trapped point in $p^{-1}\left(\left[\lambda_{0}-\varepsilon, \lambda_{0}+\varepsilon\right]\right)$ for $\varepsilon$ small enough. The proof can then be completed as in Proposition 4.1.

4.D. Quasiresonant States for Shape Resonances. We will now construct states exhibiting a time-decay behavior in a situation where shape resonances appear. It is known that in this case $\operatorname{Im} E(h)$ is of size $O\left(h^{\infty}\right)$, so this type of resonances need a separate treatment.

In this subsection we will assume that $V(x)$ is a 2-body potential which is dilation analytic in a sector $\left\{\left|x \in \mathbb{C}^{n}\right||\operatorname{Im} x| \leqq \lambda\langle\operatorname{Re} x\rangle\right\}$. We assume that $I$ is of 
the form $\left[\lambda_{0}-\varepsilon, \lambda_{0}+\varepsilon\right]$, for $\varepsilon$ small enough. Here $\lambda_{0}>0$, and there exists a connected open set $\ddot{O} \subset \mathbb{R}^{n}$ and a compact set $\mathscr{U} \subset \ddot{O}$ such that:

$$
V \leqq \lambda_{0} \text { in } \mathscr{U}, \quad V>\lambda_{0} \text { in } \ddot{O} \backslash \mathscr{U}, \quad V<\lambda_{0} \text { in } \overline{\ddot{O}}^{c} .
$$

We also assume that there are no trapped trajectories in $p^{-1}\left(\lambda_{0}\right)$ outside $\ddot{O}$.

This situation has been considered in [C-D-K-S, He-Sj2, Hi-Si]. One proves the existence of resonances due to tunneling through the potential barrier $\ddot{O} \backslash \mathscr{U}$, which are close to the real eigenvalues of a model Dirichlet problem, with an error of size $\widetilde{O}\left(e^{-2 S_{0} / h}\right)$, where $S_{0}$ is the distance between $\mathscr{U}$ and $\ddot{O}^{C}$ for the Agmon metric $\max \left(V-\lambda_{0}, 0\right) d x^{2}$ and $\tilde{O}(a)$ means $O_{\lambda}\left(e^{\left(-2 S_{0}+\lambda\right) / h}\right)$ for any $\lambda>0$.

For simplicity we will consider a case where one can obtain lower bounds (and even asymptotic expansions) on the widths of these resonances, i.e. upper bounds on their lifetimes. We will assume as in [He-Sj2, Sect. 10] that:

$$
\mathscr{U}=\left\{x_{0}\right\} \text {, where } V^{\prime \prime}\left(x_{0}\right)>0 \text {. }
$$

We will consider a resonance $E(h)$ close to an eigenvalue $E_{0}(h)$ of $P_{h}$ with Dirichlet boundary conditions in $M_{0}=B_{d}\left(\mathscr{U}, S_{0}-\eta\right)$, with $E_{0}(h) \rightarrow \lambda_{0}$ when $h \rightarrow 0$. Here $B_{d}\left(\mathscr{U}, S_{0}-\eta\right)$ is the ball around $\mathscr{U}$ of radius $S_{0}-\eta$ for the Agmon metric and $\eta \ll 1$. (see $[\mathrm{He}-\mathrm{Sj} 2]$, Theorems $10.8,10.12)$. We assume that $E_{0}(h)$ is asymptotically simple (see [He-Sj2], Theorem 10.7) and denote by $u_{h}$ the unique (modulo constant factor) resonant function associated with $E(h)$.

We can choose for example $u_{h}$ to be the eigenprojection (for the complex dilated Hamiltonian) of a normalized Dirichlet eigenfunction of $P_{h}$ for $E_{0}(h)$ (see [He-Sj2, Sect. 9]).

Then we know from [He-Sj2, Théorème 10.14] that:

$$
\Gamma(h)=-\operatorname{Im} E(h) \geqq C_{0} h^{1 / 2} e^{-2 S_{0} / h} \quad \text { for } h \text { small enough. }
$$

To formulate our result we fix a cutoff function $\chi_{0}(x) \in C_{0}^{\infty}\left(\mathbb{R}^{n}\right)$ equal to 1 in a neighborhood of $\ddot{O}$ and another cutoff function $\chi_{1}(x)$ such that no classical trajectories for $H_{p}$ in $p^{-1}\left(\lambda_{0}\right)$ starting from $\operatorname{supp} \nabla \chi_{0}$ reach $\operatorname{supp} \chi_{1}$ for positive times. It is important to notice that $\chi_{1}$ can for example be supported in the island $\ddot{O}$.

Then we have:

Theorem 4.3. With the notations above, we have:

$$
e^{-i t P_{h} / h} \chi_{0} u_{h}=e^{-i t E(h) / h} \chi_{0} u_{h}+r_{\text {out }}(t)+r_{\infty}(t),
$$

where

i) $r_{\infty}(t)$ has a norm $O\left(e^{-S_{0} / h}\right)+\tilde{O}\left(e^{-2 S_{0} / h}\right) \frac{h}{\Gamma(h)}\left(1-e^{-t \Gamma / h}\right)$.
ii) $r_{\text {out }}(t)$ satisfies the estimate:

$$
\left\|\chi_{1}(x) r_{\text {out }}(t)\right\|=O\left(h^{\infty} e^{-S_{0} / h}\right)\left(e^{-t \Gamma / h}+\frac{1}{\langle t\rangle^{n}}\right)
$$

uniformly for $t \geqq 0$. 
Proof. Let us denote by $\chi(\lambda)$ a cutoff function supported in $\left[E_{0}(h)-\lambda, E_{0}(h)+\lambda\right]$, equal to 1 in $\left[E_{0}(h)-\lambda / 2, E_{0}(h)+\lambda / 2\right]$. We first remark that:

$$
\chi_{0} u_{h}=\chi\left(P_{h}\right) \chi_{0} u_{h}+O\left(e^{-S_{0} / h}\right) \text {. }
$$

Indeed we have:

$$
\left(P_{h}-E_{0}(h)\right) \chi_{0} u_{h}=\left[P_{h}, \chi_{0}\right] u_{h}+\tilde{O}\left(e^{-2 S_{0} / h}\right) \chi_{0} u_{h}
$$

which gives for $z \in \mathbb{C} \backslash \mathbb{R}$ :

$$
\begin{aligned}
\left(P_{h}-z\right)^{-1} \chi_{0} u_{h}= & \left(E_{0}(h)-z\right)^{-1} \chi_{0} u_{h}-\left(E_{0}(h)-z\right)^{-1}\left(P_{h}-z\right)^{-1}\left[P_{h}, \chi_{0}\right] u_{h} \\
& -\left(E_{0}(h)-z\right)^{-1}\left(P_{h}-z\right)^{-1} \tilde{O}\left(e^{-2 S_{0} / h}\right) \chi_{0} u_{h} .
\end{aligned}
$$

Plugging (4.7) into formula (5.10) in Sect. 5 used to define $\chi\left(P_{h}\right) \chi_{0} u_{h}$ and using that $\left[P_{h}, \chi_{0}\right] u_{h}=O\left(e^{-S_{0} / h}\right)($ see $[\mathrm{He}-\mathrm{Sj} 2$, Théorème 10.10]) we get directly (4.6).

Now as in the proof of Theorem 3.7, we get:

$$
\begin{aligned}
e^{-i t P_{h} / h} \chi\left(P_{h}\right) \chi_{0} u_{h}= & e^{-i t E(h) / h} \chi\left(P_{h}\right) \chi_{0} u_{h} \\
& -(i h)^{-1} \int_{0}^{t} e^{-i(t-s) E(h) / h} e^{-i s P_{h} / h} \chi\left(P_{h}\right)\left[P_{h}, \chi_{0}\right] u_{h} d s .
\end{aligned}
$$

For $\delta>0, \delta \ll 1$, let us denote by $W_{\delta}(x) \in C_{0}^{\infty}(\ddot{O})$ a positive cutoff function supported in $B_{d}(\mathscr{U}, \delta)$ such that $V+W_{\delta}>\lambda_{0}$ in $\ddot{O}$. Let us put $P_{h, 1}=P_{h}+W_{\delta}$ and forget the $\delta$ dependence of $P_{h, 1}$ for the moment. We claim that:

$$
\left(\chi\left(P_{h}\right)-\chi\left(P_{h, 1}\right)\right)\left[P_{h}, \chi_{0}\right]=\tilde{O}\left(e^{-S_{0} / h}\right) .
$$

To see this, we use formula (5.10) in Sect. 5 and get:

$$
\left(\chi\left(P_{h}\right)-\chi\left(P_{h, 1}\right)\right)\left[P_{h}, \chi_{0}\right]=\frac{i}{2 \pi} \int_{\mathbb{C}} \partial_{\bar{z}} \tilde{\chi}(z)\left(z-P_{h}\right)^{-1} W_{\delta}\left(z-P_{h, 1}\right)^{-1}\left[P_{h}, \chi_{0}\right] d z \wedge d \bar{z} .
$$

Then (4.9) follows directly from Agmon-type estimates on $\left(z-P_{h, 1}\right)^{-1}$ (see for example [He-Sj2, Lemma 9.4]).

We use now formula (7.12) in Sect. 7 and get:

$$
\begin{aligned}
e^{-i t P_{h} / h} \chi\left(P_{h, 1}\right)\left[P_{h}, \chi_{0}\right]= & e^{-i t P_{h, 1} / h} \chi\left(P_{h, 1}\right)\left[P_{h}, \chi_{0}\right] \\
& -(i h)^{-1} \int_{0}^{t} e^{-i(t-s) P_{h} / h} W_{\delta} e^{-i s P_{h, 1} / h} \chi\left(P_{h, 1}\right)\left[P_{h}, \chi_{0}\right] d s .
\end{aligned}
$$

If we write:

$$
W_{\delta} e^{-i s P_{h, 1 / h}} \chi\left(P_{h, 1}\right)\left[P_{h}, \chi_{0}\right]=\int_{\mathbf{R}} e^{-i s \lambda / h} \chi(\lambda) W_{\delta} \frac{\partial E_{1}}{\partial \lambda}\left[P_{h}, \chi_{0}\right] d \lambda,
$$

where $\frac{\partial E_{1}}{\partial \lambda}$ is the spectral function of $P_{h, 1}$, we get again by Agmon-type estimates:

$$
\left\|W_{\delta} e^{-i s P_{h, 1 / h}} \chi\left(P_{h, 1}\right)\left[P_{h}, \chi_{0}\right]\right\|=\tilde{O}\left(e^{-S_{0} / h}\right) .
$$

Integrating by parts in $\lambda$ we also get:

$$
\frac{i s}{h} W_{\delta} e^{-i s P_{h, 1 / h}} \chi\left(P_{h, 1}\right)\left[P_{h}, \chi_{0}\right]=\int e^{-i s \lambda / h} W_{\delta}\left(\chi^{\prime}(\lambda) \frac{\partial E_{1}}{\partial \lambda}+\chi(\lambda) \frac{\partial^{2} E_{1}}{\partial \lambda^{2}}\right)\left[P_{h}, \chi_{0}\right] d \lambda \text {. }
$$


We now use that:

$$
\frac{\partial^{2} E_{1}}{\partial \lambda^{2}}=\frac{1}{2 i \pi}\left(R_{1}(\lambda+i 0)^{2}-R_{1}(\lambda-i 0)^{2}\right),
$$

where $R_{1}(\lambda \pm i 0)$ are the boundary values of the resolvent of $P_{h, 1}$.

We can then prove Agmon-type estimates for the kernel of $R_{1}(\lambda \pm i 0)^{2}$ in the same way as for the kernel of $R_{1}(\lambda \pm i 0)$.

So (4.10) gives:

$$
\left\|W_{\delta} e^{-i s P_{h, 1} / h} \chi\left(P_{h, 1}\right)\left[P_{h}, \chi_{0}\right]\right\|=\tilde{O}\left(\frac{1}{\langle s\rangle} e^{-S_{0} / h}\right) .
$$

Integrating by parts in $\lambda$ once more and using the same type of estimates we get that:

$$
\left\|W_{\delta} e^{-i s P_{h, 1} / h} \chi\left(P_{h, 1}\right)\left[P_{h}, \chi_{0}\right]\right\|=\tilde{O}\left(\frac{1}{\langle s\rangle^{2}} e^{-S_{0} / h}\right) .
$$

From this we get finally:

$$
\begin{aligned}
e^{-i t P_{h} / h} \chi\left(P_{h}\right) \chi_{0} u_{h}= & e^{-i t E(h) / h} \chi\left(P_{h}\right) \chi_{0} u_{h}-(i h)^{-1} \int_{0}^{t} e^{-i(t-s) E(h) / h} \\
& \cdot e^{-i s P_{h, 1} / h} \chi\left(P_{h, 1}\right)\left[P_{h}, \chi_{0}\right] u_{h} d s+\tilde{O}\left(e^{-2 S_{0} / h}\right) \frac{h}{\Gamma(h)}\left(1-e^{-t \Gamma(h) / h}\right) .
\end{aligned}
$$

Let us put now

$$
r_{\text {out }}(t)=-(i h)^{-1} \int_{0}^{t} e^{-i(t-s) E(h) / h} e^{-i s P_{h, 1 / h}} \chi\left(P_{h, 1}\right)\left[P_{h}, \chi_{0}\right] u_{h} d s .
$$

Since $\lambda_{0}$ is non-trapping for $\tilde{p}=\xi^{2}+V+W_{\delta}$, we can apply Theorem 7.2 and Egorov's Theorem to get that:

$$
\left\|\chi_{1}(x) e^{-i s P_{h, 1} / h} \chi\left(P_{h, 1}\right)\left[P_{h}, \chi_{0}\right]\right\|=\frac{O\left(h^{\infty}\right)}{\langle s\rangle^{\infty}} .
$$

To obtain (4.11) we need to take the support of $\chi(\lambda)$ sufficiently small depending on $\delta$, and use that $p=\tilde{p}$ outside $\ddot{O}$.

So it just remains to estimate $\int_{0}^{t} e^{-(t-s) \Gamma(h) / h} \frac{1}{\langle s\rangle^{n}} d s$. This is done in Appendix 2, Proposition B.1, and we get the desired estimate on $\left\|\chi_{1}(x) r_{\text {out }}(t)\right\|$.

\section{Expansion of Commutators and Localization}

5.A. Expansion of Commutators. In this subsection we generalize the commutator expansion lemma of $[\mathrm{S}-\mathrm{S}]$ to functions of operators which satisfy symbol-type estimates. We also prove a localization lemma for a function of a (selfadjoint) pseudodifferential operator. These two tools will allow us to work with functions of pseudodifferential operators as if they were themselves pseudodifferential operators (which is not clear if the pseudodifferential operators considered are not elliptic, as for example $\left.\frac{1}{2} h\left(x \cdot D_{x}+D_{x} \cdot x\right)\right)$. 
We consider first a function $F(x)$ in the symbol class $S^{n_{0}}(\mathbb{R})$, i.e.:

$$
\left|\partial_{t}^{k} F(t)\right| \leqq C_{\alpha}\langle t\rangle^{n_{0}-k}, \quad n_{0} \in \mathbb{R} .
$$

Let $H$ and $A$ be two selfadjoint operators on a Hilbert space $\mathscr{H}$, such that for any $k \in \mathbb{N}, \operatorname{ad}_{A}^{k}(H)$ is bounded. Here $\operatorname{ad}_{A}(B)=[B, A]$. In particular $H$ is bounded. From this it follows easily that $H$ and $\operatorname{ad}_{A}^{k}(H)$ are bounded on $D\left((|A|+1)^{\alpha}\right)$ for integer $\alpha$ and then for any $\alpha$ by interpolation. We will prove the following Proposition:

Proposition 5.1. The commutator $[H, F(A)]$ originally defined on $D\left((|A|+1)^{n_{0}}\right)$ can be expanded as:

$$
[H, F(A)]=\sum_{k=1}^{n-1} \frac{1}{k !} F^{(k)}(A) \operatorname{ad}_{A}^{k}(H)+R_{n}(A, H, F),
$$

where $R_{n}(A, H, F)$ is bounded on $\mathscr{H}$ with a norm bounded by $C_{n}\left\|\operatorname{ad}_{A}^{n}(H)\right\|$, for $n>n_{0}+1$, and for a constant $C_{n}$ depending only on a finite number of seminorms of $F$ in $S^{n_{0}}(\mathbb{R})$.

Proof. We write $F=\left(x^{2}+1\right)^{n_{1}} g=f g$, with $n_{0}-2 n_{1}=-1-\varepsilon, \varepsilon>0$. Then $g \in S^{-1-\varepsilon}(\mathbb{R})$, from which it follows that $\forall n \in \mathbb{N}, s^{n} \hat{g}(s) \in L^{\infty}(\mathbb{R})$, and hence also $s^{n} \hat{g}(s) \in L^{1}(\mathbb{R})$.

Hence we can apply to $g(A)$ the result of $[\mathrm{S}-\mathrm{S}$, Lemma 1.1]. We will write:

$$
\left[H,\left(A^{2}+1\right)^{n_{1}} g(A)\right]=\left[H,\left(A^{2}+1\right)^{n_{1}}\right] g(A)+\left(A^{2}+1\right)^{n_{1}}[H, g(A)] .
$$

It is straightforward to show by induction on the degree of $f$, that if $f(s)$ is a polynomial of degree $n_{0}$, one has:

$$
[H, f(A)]=\sum_{k=1}^{n_{0}} \frac{f^{(k)}}{k !}(A) \operatorname{ad}_{A}^{k}(H)
$$

So

$$
\left[H,\left(A^{2}+1\right)^{n_{1}}\right] g(A)=\sum_{k=1}^{2 n_{1}} \frac{f^{(k)}}{k !}(A) \operatorname{ad}_{A}^{k}(H) g(A) .
$$

We apply now $[\mathrm{S}-\mathrm{S}$, Lemma 1.1$]$ to $\tilde{H}=\operatorname{ad}_{A}^{k}(H)$ (since $\tilde{H}$ is either selfadjoint or antiselfadjoint). We get:

$$
\left[H,\left(A^{2}+1\right)^{n_{1}} g(A)\right]=\sum_{n=1}^{2 n_{1}} \sum_{k=0}^{M-1} \frac{f^{(n)}}{n !}(A) \frac{g^{(k)}}{k !}(A) \operatorname{ad}_{A}^{n+k}(H)+\frac{f^{(n)}}{n !}(A) R_{M}\left(A, \operatorname{ad}_{A}^{n}(H), g\right) .
$$

The second term in the r.h.s. of (5.1) is:

$$
\sum_{k=1}^{M-1} f(A) \frac{g^{(k)}}{k !}(A) \operatorname{ad}_{A}^{k}(H)+f(A) R_{M}(A, H, g) .
$$

Let us take in (5.2) $M=M(n)$ such that $n+M(n)=M_{0} \gg 1$. We get from (5.2), (5.3):

$$
\begin{aligned}
{[H, F(A)] } & =\sum_{n=0}^{2 n_{1}} \sum_{\substack{k=0 \\
n+k \geqq 1}}^{M_{0}-1-n} \frac{f^{(n)}}{n !}(A) \frac{g^{(k)}}{k !}(A) \operatorname{ad}_{A}^{n+k}(H)+R_{M_{0}} \\
& =\sum_{k=1}^{M_{0}-1} \frac{(f g)^{(k)}}{k !}(A) \operatorname{ad}_{A}^{k}(H)+R_{M_{0}}
\end{aligned}
$$


by the binomial formula. It remains to estimate $R_{M_{0}}$.

Let us first consider a term of the type:

$$
f^{(n)}(A) R_{M_{0}-n}\left(A, \operatorname{ad}_{A}^{n}(H), g\right)=\int_{-\infty}^{+\infty} f^{(n)}(A) e^{i A s} K_{n}(s) d s,
$$

where

$$
K_{n}(s)=\hat{g}(s) \int_{0}^{s} d u_{1} \cdots \int_{0}^{u_{M_{0}-n-1}} d u_{M_{0}-n} e^{-i A u_{M_{0}-n}} \operatorname{ad}_{A}^{M_{0}}(H) e^{i A u_{M_{0}-n}}
$$

(see [S-S], Eqs. (1.12), (1.10)).

We use now that $f^{(n)}(A)=\sum_{k=0}^{2 n_{1}-n} c_{n, k} A^{k}$. Let us consider one term of (5.4) with $f^{(n)}(A)$ replaced by $A^{k}, k \leqq 2 n_{1}-n$. We get:

$$
I_{n, k}=\int_{-\infty}^{+\infty}\left(\frac{1}{i} \frac{\partial}{\partial s}\right)^{k} e^{i A s} K_{n}(s) d s
$$

where the integral converges in norm in $\mathscr{L}\left(D((|A|+1))^{k}, \mathscr{H}\right)$.

Since $K_{n}(s)$ has a norm in $\mathscr{L}\left(D\left((|A|+1)^{k}\right), D\left((|A|+1)^{k}\right)\right)$ bounded by $C_{n}|\hat{g}(s)| s^{M_{0}-n}$ we can integrate by parts once.

Then we have:

$$
\partial_{s}^{l} K_{n}^{\prime}(s)=\sum_{l_{1}=0}^{\ell} \partial_{s}^{l_{1}} \hat{g}(s) O\left(s^{M_{0}-n+\ell_{1}-\ell}\right),
$$

where $O(\quad)$ means in norm in $\mathscr{L}\left(D\left((|A|+1)^{k}\right), D\left((|A|+1)^{k}\right)\right)$.

We use now that $s^{M_{0}-n+\ell_{1}-\ell} \partial_{s}^{\ell_{1}} \hat{g}(s)$ is the Fourier transform of $D_{t}^{M_{0}-n+\ell_{1}-\ell}(i t)^{\ell_{1}} g(t)$ which is in $S^{\ell+n-M_{0}-(1+\varepsilon)}(\mathbb{R})$.

Since $l \leqq 2 n_{1}-n$, by taking $M_{0}>2 n_{1}, s^{M_{0}-n+\ell_{1}-\ell} \partial_{s}^{\ell_{1}} \hat{g}(s)$ is the Fourier transform of a term in $S^{-1-\varepsilon}(\mathbb{R})$, so tends to zero at infinity faster than any power. So we can integrate by parts $k$ times in (5.5) and we get that $I_{n, k}$ has a norm bounded by $C_{M_{0}}\left\|\operatorname{ad}_{A}^{M_{0}}(H)\right\|$.

Here $C_{M_{0}}$ depends only on a finite number of seminorms of $F(s)$ in $S^{n_{0}}(\mathbb{R})$. This completes the proof of the proposition.

5.B. A Localization Result. We now prove a localization result for functions of pseudodifferential operators. We consider a real function $A(x, \xi)$ in some symbol class $S\left(m_{0}, g_{0}\right)$ for some $\sigma$-temperate metric $g_{0}$ and some $g_{0}$-temperate weight $m_{0}$. We assume that $A\left(x, h D_{x}\right)$ is essentially selfadjoint on $S\left(\mathbb{R}^{n}\right)$ and denote by $A\left(x, h D_{x}\right)$ its unique selfadjoint extension.

We have the following result:

Proposition 5.2. Let $\chi_{1}(x, \xi) \in C_{0}^{\infty}\left(T^{*} \mathbb{R}^{n}\right)$ and such that on $\operatorname{supp} \chi_{1}$ one has:

$$
C_{0} \leqq A(x, \xi) \leqq C_{1} \text {. }
$$

Let $f(\lambda) \in S^{0}(\mathbb{R})$ such that:

Then

$$
f(\lambda)=0 \text { for } C_{0}-\varepsilon_{0} \leqq \lambda \leqq C_{1}+\varepsilon_{0}, \quad \varepsilon_{0}>0 .
$$

$$
f\left(A\left(x, h D_{x}\right)\right) \chi_{1}\left(x, h D_{x}\right) \text { has a norm } O\left(h^{\infty}\right) .
$$


Proof. Let $\chi(\lambda)$ be a cutoff function supported in $\left[C_{0}-\varepsilon_{0} / 2, C_{1}+\varepsilon_{0} / 2\right]$, equal to 1 in $\left[C_{0}-\varepsilon_{0} / 3, C_{1}+\varepsilon_{0} / 3\right]$. Then $\chi(A) f(A)=0$, so it suffices to show that $\chi_{1}\left(x, h D_{x}\right)-$ $\chi(A) \chi_{1}\left(x, h D_{x}\right)=O\left(h^{\infty}\right)$. Let us now take a function $A_{1}(x, \xi)$ such that:

$$
\begin{gathered}
A_{1}=A \text { in a neighborhood of supp } \chi_{1}, \\
C_{0}-\frac{\varepsilon_{0}}{4} \leqq A_{1} \leqq C_{1}+\frac{\varepsilon_{0}}{4}, \\
A_{1}(x, \xi) \text { is constant at infinity. }
\end{gathered}
$$

We denote by $\tilde{A}\left(x, h D_{x}\right)$ its Weyl quantization which is a bounded operator by (5.8).

By (5.7) we get that for $h$ small enough:

$$
C_{1}-\frac{\varepsilon_{0}}{3} \leqq A_{1}\left(x, h D_{x}\right) \leqq C_{1}+\frac{\varepsilon_{0}}{3} .
$$

So $\chi\left(A_{1}\right)=1$ and we are left with the proof of:

$$
\left(\chi\left(A_{1}\right)-\chi(A)\right) \chi_{1}\left(x, h D_{x}\right)=O\left(h^{\infty}\right) .
$$

This is proven in Lemma 5.3 below, which will complete the proof of the proposition.

Lemma 5.3. Let $\chi_{1}$ be as in Proposition 5.2 and $\chi$ as in the proof of Proposition 5.2. Then:

$$
\left(\chi\left(A_{1}\right)-\chi(A)\right) \chi_{1}\left(x, h D_{x}\right)=O\left(h^{\infty}\right) .
$$

Proof. We use a formula for functional calculus due to Helffer-Sjöstrand [He-Sj3]. We denote by $\tilde{\chi}(z)$ an almost analytic extension of $\chi(\lambda)$, i.e. a smooth function on $\mathbb{C}$ such that

$$
\partial_{\bar{z}} \tilde{\chi}(z)=O\left(|\operatorname{Im} z|^{\infty}\right) .
$$

Moreover we can take $\tilde{\chi}(z)$ with compact support in $\mathbb{C}$.

Then one can prove (see $[\mathrm{He}-\mathrm{Sj} 3]$ ) that:

$$
\chi(A)=\frac{i}{2 \pi} \int_{\mathbb{C}} \partial_{\bar{z}} \tilde{\chi}(z)(z-A)^{-1} d z \wedge d \bar{z} .
$$

The integral in (5.10) converges in norm by (5.9). (5.10):

Using that $(z-A)^{-1}-\left(z-A_{1}\right)^{-1}=(z-A)^{-1}\left(A-A_{1}\right)\left(z-A_{1}\right)^{-1}$, we get by

$$
\left(\chi(A)-\chi\left(A_{1}\right)\right) \chi_{1}=\frac{i}{2 \pi} \int_{\mathbb{C}} \partial_{\bar{z}} \tilde{\chi}(z-A)^{-1}\left(A-A_{1}\right)\left(z-A_{1}\right)^{-1} \chi_{1} d z \wedge d \bar{z}
$$

Then we have:

$$
\begin{aligned}
\left(A-A_{1}\right)\left(z-A_{1}\right)^{-1} \chi_{1}= & \left(A-A_{1}\right) \chi_{1}\left(z-A_{1}\right)^{-1} \\
& +\left(A-A_{1}\right)\left(z-A_{1}\right)^{-1}\left[A_{1}, \chi_{1}\right]\left(z-A_{1}\right)^{-1} .
\end{aligned}
$$

By pseudodifferential calculus $\left(A-A_{1}\right) \chi_{1}$ has a norm $O\left(h^{\infty}\right)$ and $\left[A_{1}, \chi_{1}\right]$ has a 
norm $O(h)$ so $\left(\chi(A)-\chi\left(A_{1}\right)\right) \chi_{1}=O(h)$ using that $\left\|(B-z)^{-1}\right\|_{\mathscr{L}(\mathscr{H}, D(B))}=O\left((\operatorname{Im} z)^{-1}\right)$ for any selfadjoint operator $B$ on a Hilbert space $\mathscr{H}$.

We use now that:

$$
\begin{aligned}
& \left(A-A_{1}\right)\left(z-A_{1}\right)^{-1}\left[A_{1}, \chi_{1}\right]\left(z-A_{1}\right)^{-1} \\
& \quad=\left(A-A_{1}\right)\left[A_{1}, \chi_{1}\right]\left(z-A_{1}\right)^{-2}+\left(A-A_{1}\right)\left(z-A_{1}\right)^{-1}\left[A_{1},\left[A_{1}, \chi_{1}\right]\right]\left(z-A_{1}\right)^{-1} .
\end{aligned}
$$

Again by pseudodifferential calculus $\left(A-A_{1}\right)\left[A_{1}, \chi_{1}\right]$ has a norm $O\left(h^{\infty}\right)$ and $\left[A_{1},\left[A_{1}, \chi_{1}\right]\right]$ a norm $O\left(h^{2}\right)$ so $\left[\chi(A)-\chi\left(A_{1}\right)\right] \chi_{1}=O\left(h^{2}\right)$. Iterating this argument in the obvious way gives the Lemma.

5.C. A Result on Functional Calculus for Singular Potentials. In this subsection, we consider a Schrödinger operator satisfying the hypotheses of Sect. 2. We will show that outside the singular support of $V_{s}$, for a smooth cutoff function $f \in C_{0}^{\infty}(\mathbb{R})$, the operator $f\left(P_{h}\right)$ given by the functional calculus is a pseudodifferential operator modulo some error of size $O\left(h^{\infty}\right)$. This result is summarized in the next proposition:

Proposition 5.4. Let $\chi_{0}(x, \xi) \in C_{0}^{\infty}\left(T^{*}\left(\mathbb{R}^{n}\right)\right)$ such that $V_{s}$ is smooth on support of $\chi_{0}$. Let us take a smooth potential $V_{1}$ satisfying the same hypotheses as $V_{r}$, such that $V_{1}=V_{s}(x)+V_{r}(x)$ on support of $\chi_{0}(x, \xi)$. Then if $P_{h, 1}=-h^{2} \Delta_{x}+V_{1}$, for any smooth cutoff function $\chi \in C_{0}^{\infty}(\mathbb{R})$, we have:

$$
\left(\chi\left(P_{h, 1}\right)-\chi\left(P_{h}\right)\right) \chi_{0}\left(x, h D_{x}\right)=O_{H^{2}}\left(h^{\infty}\right),
$$

where $O_{H^{2}}$ means an estimate in $\mathscr{L}\left(L^{2}, H^{2}\right)$.

Proof. Without loss of generality, we can replace $\operatorname{sing} \operatorname{supp} V_{s}$ by $\operatorname{supp} V_{s}$. Let us take another smooth cutoff function $\chi_{1}(x) \in C_{0}^{\infty}\left(\mathbb{R}^{n}\right)$, such that:

$$
\chi_{1}(x) \chi_{0}(x, \xi)=\chi_{0}(x, \xi) \text {, }
$$

and $V_{1}=V_{s}(x)+V_{r}(x)$ on support of $\chi_{1}(x)$. Since $\chi_{1}(x) \chi_{0}\left(x, h D_{x}\right)=\chi_{0}\left(x, h D_{x}\right)+O\left(h^{\infty}\right)$ by pseudodifferential calculus, it suffices to show that:

$$
\left(\chi\left(P_{h, 1}\right)-\chi\left(P_{h}\right)\right) \chi_{1}(x)=O_{H^{2}}\left(h^{\infty}\right) .
$$

To do this we use formula $(5.10)$ to represent $\chi\left(P_{h}\right)$. One has:

$$
\chi\left(P_{h}\right)=\frac{i}{2 \pi} \int_{\mathbb{C}} \frac{\partial \tilde{\chi}(z)}{\partial \bar{z}}\left(z-P_{h}\right)^{-1} d z \wedge d \bar{z} .
$$

Here $\tilde{\chi}(z)$ is as in (5.10) an almost analytic extension of $\chi(\lambda)$ with compact support in $\mathbb{C}$. This formula gives:

$$
\left(\chi\left(P_{h}\right)-\chi\left(P_{h, 1}\right)\right) \chi_{1}(x)=\frac{i}{2 \pi} \int_{\mathbb{C}} \frac{\partial \tilde{\chi}(z)}{\partial \bar{z}}\left(\left(z-P_{h}\right)^{-1}-\left(z-P_{h, 1}\right)^{-1}\right) \chi_{1} d z \wedge d \bar{z}
$$

Then we have:

$$
\begin{aligned}
\left(\left(z-P_{h}\right)^{-1}-\left(z-P_{h, 1}\right)^{-1}\right) \chi_{1} & =\left(z-P_{h}\right)^{-1}\left(P_{h}-P_{h, 1}\right)\left(z-P_{h, 1}\right)^{-1} \chi_{1} \\
& =\left(z-P_{h}\right)^{-1} W\left(z-P_{h, 1}\right)^{-1} \chi_{1},
\end{aligned}
$$

where $W=V_{r}+V_{s}-V_{1}$ is supported where $\chi_{1}=0$. Then we have:

$$
W\left(z-P_{h, 1}\right)^{-1} \chi_{1}=W\left(z-P_{h, 1}\right)^{-1}\left[P_{h, 1}, \chi_{1}\right]\left(z-P_{h, 1}\right)^{-1}=O\left(\frac{h}{(\operatorname{Im} z)^{2}}\right) \text {. }
$$


This can be iterated since $\left[P_{h, 1}, \chi_{1}\right]=\chi_{2}(x)\left[P_{h, 1}, \chi_{1}\right]$, where $\chi_{2}$ is another cutoff with $W \chi_{2}=0$. This way we get finally a term in the integrand of (5.13) of norm between $L^{2}$ and $H^{2}$ equal to $O\left(h^{N}(\operatorname{Im} z)^{-N}\right)$ for any $N$, which completes the proof of the proposition since $\partial_{\bar{z}} \tilde{\chi}(z)=O(\operatorname{Im} z)^{\infty}$.

\section{Semiclassical Propagation Estimates}

In this section we prove a semiclassical version of the propagation estimates of Sigal-Soffer [S-S]. Since we will later apply it to different types of conjugate operators, we will prove the propagation estimates in an abstract setting.

We consider a self adjoint operator $H$ on an Hilbert space $\mathscr{H}$ with domain $D(H) . H$ is supposed to depend on the Planck constant $h$. Actually the only place where $h$ will appear will be in the size of the multicommutators. We consider also a commutative family $A(t)$ of unbounded $h$-dependent self adjoint operators with a common domain $D$ satisfying the following properties: for a cutoff function $\chi_{0}(\lambda) \in C_{0}^{\infty}(\mathbb{R})$ we denote by $H_{1}$ the bounded operator $\chi_{0}(H) H$. We fix another cutoff function $\chi_{1}(\lambda)$ such that $\chi_{0} \chi_{1}=\chi_{1}$.

We denote by $D A(t)$ the Heisenberg derivative of $A(t)$ w.r.t. $H_{1}$, i.e.

$$
D A(t)=i h^{-1}\left[H_{1}, A(t)\right]+\frac{d A}{d t}
$$

and by $\left(\tilde{\mathrm{ad}}_{A}^{k}\right)(H)$ the multicommutator $h^{-k}[[H, A] \cdots A]$. With these notations we ask that $A(t)$ satisfies:

(H.2.i) $A(t)$ is norm differentiable with $\frac{d A}{d t}$ uniformly bounded in $t$.

(H.2.ii) $\chi_{1}(H) D A(t) \chi_{1}(H) \geqq C_{0} \chi_{1}^{2}(H)$ for some $C_{0}>0$.

(H.2.iii) $\operatorname{ad}_{A(t)}^{k}(H)$ are uniformly bounded in $t$ and $h$.

(H.2.iv) $\frac{d A}{d t}$ commutes with $A$.

This last assumption is not necessary and can be replaced by control on the multicommutators $\tilde{\mathrm{ad}}_{A(t)}^{\mathrm{k}}\left(\frac{d A}{d t}\right)$.

As in [S-S], we set $A(0)=A$ and $\tau=t+1$.

We will consider the propagation observables

$$
\Phi_{\alpha}=\Phi_{\alpha}(t)=-(-A(t))^{\alpha} F\left(\frac{A(t)}{\tau} \leqq-\varepsilon\right),
$$

where $F(\Omega)$ stands for a smoothed-out characteristic function of $\Omega$ with $F^{\prime} \leqq 0$. We also fix a bounded operator $\chi_{\text {out }}$ such that:

$$
\Phi_{\alpha}(0) \chi_{1}(H) \chi_{\text {out }} \text { is bounded with norm } O\left(h^{\infty}\right) \text { for any } \alpha \in \mathbb{N} \text {. }
$$

Later on $\chi_{\text {out }}$ will be a pseudodifferential cutoff with support in an outgoing region.

We will prove the following theorem, which for $h=1$ is an extension of Theorems 2.3-2.5 in [S-S]. 
Theorem 6.1. Under the hypotheses (H.2), $\forall \alpha, \forall u \in \mathscr{H}$, we have:
i) $\int_{0}^{+\infty}\left\langle\Phi_{\alpha}^{2}(t) e^{-i t H / h} \chi_{\text {out }} u, e^{-i t H / h} \chi_{\text {out }} u\right\rangle d t \leqq C_{N} h^{N}\|u\|^{2} \quad \forall N \in \mathbb{N}$,
ii) $\left\|\Phi_{\alpha}(t) e^{-i t H / h} \chi_{\text {out }} u\right\| \leqq C_{N} h^{N}\|u\| \quad \forall N \in \mathbb{N}$, uniformly in $t \geqq 0$,
iii) $\left\|F\left(\frac{A(t)}{\tau} \leqq-\varepsilon\right) e^{-i t H / h} \chi_{\text {out }} u\right\| \leqq C_{N} \frac{h^{N}}{\langle t\rangle^{N}}\|u\| \quad \forall N \in \mathbb{N}$, uniformly in $t \geqq 0$.

We start by fixing some notations.
We will denote by $F_{\delta}$ any operator of the form $f_{\delta}(A(t), t) F\left(\frac{A(t)}{\tau} \leqq-\varepsilon\right)$, where $f_{\delta}(\lambda, t)$ belongs to the symbol class $S^{\delta}\left(\mathbb{R}_{\lambda}\right)$ uniformly in $t$. If $f_{\delta}$ is such that $f_{\delta}(\lambda, t) \geqq$ $C\langle\lambda\rangle^{\delta}$, we will denote $f_{\delta}(A(t), t) F\left(\frac{A(t)}{\tau} \leqq-\varepsilon\right)$ by $F_{\delta}^{+}$. We will denote by $G$ the operator $F\left(\frac{A(t)}{\tau} \leqq-\varepsilon / 2\right)$, and put

$$
\Phi_{\alpha}^{\prime}=\frac{\partial \Phi_{\alpha}}{\partial \lambda}, \frac{d \Phi_{\alpha}}{d t}=\frac{\partial \Phi_{\alpha}}{\partial t}(A(t), t)
$$

where $\Phi_{\alpha}(\lambda, t)=-(-\lambda)^{\alpha} F\left(\frac{\lambda}{\tau} \leqq-\varepsilon\right)$.

We take a vector $u$ in $\mathscr{H}$ and denote by $\psi_{t}$ the vector $e^{-i t H_{1 / h}} \chi_{1}(H) \chi_{\text {out }} u$. If $B$ is an observable, we denote by $\langle B\rangle$ the expectation value $\left\langle B \psi_{t}, \psi_{t}\right\rangle$. We first prove the following lemma:

Lemma 6.2. For $\alpha<N_{0}+1,0<\beta, 0<\beta^{\prime}<1$, we have:

$$
\left\langle D \Phi_{\alpha}\right\rangle \geqq C_{0}\left\langle\chi_{1}(H) F_{\alpha-1}^{+} \chi_{1}(H)\right\rangle+R_{t}
$$

where

$$
\begin{aligned}
\left|R_{t}\right| \leqq & C_{1} \sum_{k=2}^{N_{0}} \sum_{\ell=0}^{M} h^{k+\ell-1}\left\|F_{\alpha-k-\beta} \psi_{t}\right\|\left\|F_{\beta-\ell} \psi_{t}\right\| \\
& +C_{1} \sum_{k=2}^{N_{0}} h^{k+M} \tau^{\beta-(M+1)}\left\|F_{\alpha-k-\beta} \psi_{t}\right\|\left\|\psi_{0}\right\| \\
& +C_{1} \sum_{k=1}^{\left[N_{0} / 2\right]+1} h^{k}\left\|F_{((\alpha-1) / 2)-k+\beta^{\prime}} \psi_{t}\right\|\left\|F_{((\alpha-1) / 2)-\beta^{\prime}} \psi_{t}\right\| \\
& +C_{1} h^{\left[N_{0} / 2\right]+2} \tau^{(\alpha-1) / 2-\left[N_{0} / 2\right]-2}\left\|F_{(\alpha-1) / 2} \psi_{t}\right\|\left\|\psi_{0}\right\| \\
& +C_{1} h^{N_{0}} \tau^{\alpha-\left(N_{0}+1\right)}\left\|G \psi_{t}\right\|\left\|\psi_{0}\right\| .
\end{aligned}
$$

We will follow closely the proof of [S-S]. The proof is divided in several steps.

Step 1: in Step 1 we compute $D \Phi_{\alpha}$. We start by computing $d_{t} \Phi_{\alpha}$. Since $\tau=t+1$, we have:

$$
\frac{d \Phi_{\alpha}}{d t}=\Phi_{\alpha}^{\prime} \frac{d A}{d t}+(-A)^{\alpha} \frac{A(t)}{\tau^{2}} F^{\prime}\left(\frac{A(t)}{\tau} \leqq-\varepsilon\right) \geqq \Phi_{\alpha}^{\prime} \frac{d A}{d t}
$$


Let us now compute $i h^{-1}\left[H_{1}, \Phi_{\alpha}\right]$. Since $\Phi_{\alpha}=\Phi_{\alpha} G$, we have:

$$
i h^{-1}\left[H_{1}, \Phi_{\alpha}\right]=i h^{-1}\left[H_{1}, \Phi_{\alpha}\right] G+i h^{-1} \Phi_{\alpha}\left[H_{1}, G\right] \text {. }
$$

Using the commutator expansion of Proposition 5.1, we get:

$i h^{-1}\left[H_{1}, \Phi_{\alpha}\right]=i h^{-1} \Phi_{\alpha}^{\prime}\left[H_{1}, A\right]+\sum_{k=2}^{N_{0}} F_{\alpha-k} h^{k-1}\left(\widetilde{\mathrm{ad}_{A}}\right)^{k}\left(H_{1}\right)+i h^{-1} R_{N_{0}+1}\left(A, H_{1}, \Phi_{\alpha}\right)$

for $N_{0} \geqq \sup (2, \alpha)$.

Applying again the commutator expansion to $\left[H_{1}, G\right]$, and using that:

$$
F(\lambda \leqq-\varepsilon) G^{(k)}(\lambda \leqq-\varepsilon / 2)=0 \text { for } k \geqq 1,
$$

we get:

$$
i h^{-1} \Phi_{\alpha}\left[H_{1}, G\right]=i h^{-1} \Phi_{\alpha} R_{N_{0}+1}\left(A, H_{1}, G\right) .
$$

From (6.3)...(6.5), we get:

$$
\begin{aligned}
D \Phi_{\alpha} \geqq & \Phi_{\alpha}^{\prime} D A(t)+\sum_{k=2}^{N_{0}} F_{\alpha-k} h^{k-1}\left(\tilde{\mathrm{ad}}_{A}\right)^{k}\left(H_{1}\right) G+h^{-1} R_{N_{0}+1}\left(A, H_{1}, \Phi_{\alpha}\right) G \\
& +h^{-1} \Phi_{\alpha} R_{N_{0}+1}\left(A, H_{1}, G\right) .
\end{aligned}
$$

To complete Step 1 we will estimate the size of the remainder terms. We have:

$$
\begin{aligned}
\left|\left\langle h^{-1} R_{N_{0}+1}\left(A, H_{1}, \Phi_{\alpha}\right) G\right\rangle\right| & \leqq h^{-1}\left\|R_{N_{0}+1}\left(A, H_{1}, \Phi_{\alpha}\right)\right\|\left\|G \psi_{t}\right\|\left\|\psi_{0}\right\| \\
& =O\left(h^{N_{0}} \tau^{\alpha-\left(N_{0}+1\right)}\right)\left\|G \psi_{t}\right\|\left\|\psi_{0}\right\| .
\end{aligned}
$$

This follows from Proposition 5.1 with $A$ replaced by $\tilde{A}=\frac{A}{\tau}$ if one recalls that $\Phi_{\alpha}(\lambda, t)=(-\lambda)^{\alpha} F\left(\frac{\lambda}{\tau} \leqq-\varepsilon\right)$.

Similarly we have:

$$
\begin{aligned}
\left|\left\langle\Phi_{\alpha} h^{-1} R_{N_{0}+1}\left(A, H_{1}, G\right)\right\rangle\right| \\
\quad=\left|\left\langle\Phi_{\alpha} h^{-1} R_{N_{0}+1}\left(A, H_{1}, G\right) \psi_{t}, \psi_{t}\right\rangle\right| \\
\quad=\left|\left\langle(-A(t))^{N_{0}+1} R_{N_{0}+1}\left(A, H_{1}, G\right) \psi_{t},(-A(t))^{-\left(N_{0}+1\right)} \Phi_{\alpha} \psi_{t}\right\rangle\right| .
\end{aligned}
$$

Again by Proposition 5.1 we have $\left\|h^{-1}(-A(t))^{N_{0}+1} R_{N_{0}+1}\left(A, H_{1}, G\right)\right\|=O\left(h^{N_{0}}\right)$ and since $\alpha-N_{0}-1<0$, we have:

$$
\left\|(-A(t))^{-\left(N_{0}+1\right)} \Phi_{\alpha} \psi_{t}\right\|=O\left(\tau^{\alpha-\left(N_{0}+1\right)}\right)\left\|G \psi_{t}\right\| .
$$

So the remainder terms in (6.6) have expectation values bounded by

$$
h^{N_{0}} \tau^{\alpha-\left(N_{0}+1\right)}\left\|G \psi_{t}\right\|\left\|\psi_{0}\right\| \text {. }
$$

Step 2. We estimate the terms $F_{\dot{\alpha}-k}\left(\widetilde{\operatorname{ad}}_{A}\right)^{k}\left(H_{1}\right) G$. We will use the trick of SigalSoffer of commuting $(-A(t))^{\beta} G$ through $\left(\operatorname{ad}_{A(t)}\right)^{k}\left(H_{1}\right)$, but in our case we need to take $\beta>1$. We write:

$$
\begin{aligned}
F_{\alpha-k}\left(\tilde{\operatorname{ad}}_{A(t)}\right)^{k}\left(H_{1}\right) G & =F_{\alpha-k}(-A)^{-\beta}(-A)^{\beta} G\left(\tilde{\operatorname{ad}}_{A(t)}\right)^{k}\left(H_{1}\right) G \\
& =F_{\alpha-k-\beta}\left(\tilde{\operatorname{ad}}_{A(t)}\right)^{k}\left(H_{1}\right) F_{\beta}+F_{\alpha-k-\beta}\left[F_{\beta},\left(\tilde{\operatorname{ad}}_{A(t)}\right)^{k}\left(H_{1}\right)\right] G .
\end{aligned}
$$


We use again Proposition 5.1 to expand $\left[F_{\beta},\left(\tilde{a d}_{A(t)}\right)^{k}\left(H_{1}\right)\right]$, but this time by putting the functions of $A(t)$ to the right. (This follows directly from Proposition 5.1 by taking adjoints.)

We get:

$$
\left[F_{\beta},\left(\tilde{\mathrm{ad}}_{A(t)}\right)^{k}\left(H_{1}\right)\right]=\sum_{\ell=1}^{M} h^{\ell}\left(\tilde{\mathrm{ad}}_{A(t)}\right)^{k+\ell}\left(H_{1}\right) F_{\beta-\ell}+O\left(h^{M+1} \tau^{\beta-(M+1)}\right)
$$

So we finally have:

$S_{k} \stackrel{\text { def }}{=} h^{k-1} F_{\alpha-k}\left(\widetilde{\mathrm{ad}_{A(t)}}\right)^{k}\left(H_{1}\right) G=\sum_{\ell=0}^{M} h^{k+\ell-1} F_{\alpha-k-\beta}\left(\widetilde{a d}_{A(t)}\right)^{k+\ell}\left(H_{1}\right) F_{\beta-\ell}+R_{1, k}$,

where

$$
\left\langle R_{1, k}\right\rangle=O\left(h^{k+M} \tau^{\beta-(M+1)}\right)\left\|F_{\alpha-k-\beta} \psi_{t}\right\|\left\|\psi_{0}\right\| .
$$

Step 3. We symmetrize the term $\Phi_{\alpha}^{\prime} D A(t)$, and use the positivity of $D A(t)$. Since $\Phi_{\alpha}^{\prime}(\lambda, t) \geqq C\langle\lambda\rangle^{\alpha-1} F\left(\frac{\lambda}{\tau} \leqq-\varepsilon\right)$, we write:

$$
\Phi_{\alpha}^{\prime} D A(t) G=F_{(\alpha-1) / 2}^{+} D A(t) F_{(\alpha-1) / 2}^{+}+F_{(\alpha-1) / 2}\left[F_{(\alpha-1) / 2}, D A(t)\right] G .
$$

Using also that

$$
\left\langle\Phi_{\alpha}^{\prime} D A(t) G\right\rangle=\left\langle\chi_{1}(H) \Phi_{\alpha}^{\prime} D A(t) G \chi_{1}(H)\right\rangle
$$

we get:

$$
\begin{aligned}
\left\langle\Phi_{\alpha}^{\prime} D A(t) G\right\rangle \\
=\left\langle F_{(\alpha-1) / 2} \chi_{1}(H) D A(t) \chi_{1}(H) F_{(\alpha-1) / 2}\right\rangle+\left\langle\left[\chi_{1}(H), F_{(\alpha-1) / 2}\right] D A(t) F_{(\alpha-1) / 2} \chi_{1}(H)\right\rangle \\
\quad+\left\langle F_{(\alpha-1) / 2} \chi_{1}(H) D A(t)\left[\chi_{1}(H), F_{(\alpha-1) / 2}\right]\right\rangle+\left\langle\chi_{1}(H)\left[F_{(\alpha-1) / 2}, D A(t)\right] G \chi_{1}(H)\right\rangle \\
=\left\langle F_{(\alpha-1) / 2} \chi_{1}(H) D A(t) \chi_{1}(H) F_{(\alpha-1) / 2}\right\rangle+\left\langle K_{1}\right\rangle+\left\langle K_{2}\right\rangle+\left\langle K_{3}\right\rangle .
\end{aligned}
$$

Let us first compute $K_{1}$.

We have:

$$
\begin{aligned}
K_{1}= & {\left[\chi_{1}(H), F_{(\alpha-1) / 2}\right](-A(t))^{\beta^{\prime}} G D A(t) F_{(\alpha-1) / 2}(-A(t))^{-\beta^{\prime}} \chi_{1}(H) } \\
& +\left[\chi_{1}(H), F_{(\alpha-1) / 2}\right]\left[(-A(t))^{\beta^{\prime}} G, D A(t)\right] F_{(\alpha-1) / 2}(-A(t))^{-\beta^{\prime}} \chi_{1}(H) \\
= & K_{11}+K_{12} .
\end{aligned}
$$

Let us start by computing $\left[F_{(\alpha-1) / 2}, \chi_{1}(H)\right]$ : again by Proposition 5.1, we get:

$$
\begin{aligned}
{\left[F_{(\alpha-1) / 2}, \chi_{1}(H)\right]=} & \sum_{1}^{\left[N_{0} / 2\right]+1} F_{((\alpha-1) / 2)-k} h^{k}\left(\tilde{a d}_{A(t)}\right)^{k}\left(\chi_{1}(H)\right) \\
& +O\left(h^{\left[N_{0} / 2\right]+2} \tau^{(\alpha-1) / 2-\left(\left[N_{0} / 2\right]+2\right)}\right) .
\end{aligned}
$$

In (6.12) we use that $\frac{\alpha-1}{2}<N_{0} / 2$. In (6.11), we take $\beta^{\prime}<1$ and use (see [S-S, Lemma A.2]), that:

$$
\left[-A(t)^{\beta^{\prime}} G, D A(t)\right]=O(h)
$$


From (6.12) we get:

$$
\begin{aligned}
K_{11}= & \sum_{k=1}^{\left[N_{0} / 2\right]+1} h^{k} F_{((\alpha-1) / 2)-k+\beta^{\prime}} B_{k} F_{((\alpha-1) / 2)-\beta^{\prime}} \\
& +h^{\left[N_{0} / 2\right]+2} \tau^{(\alpha-1) / 2-\left[N_{0} / 2\right]-2} R_{N_{0}} F_{(\alpha-1) / 2},
\end{aligned}
$$

where $B_{k}$ and $R_{N_{0}}$ are bounded operators, uniformly in $h$ and $t$. Similarly we get:

$$
\begin{aligned}
K_{12}= & \sum_{1}^{\left[N_{0} / 2\right]+1} h^{k+1} F_{((\alpha-1) / 2)-k} B_{k} F_{((\alpha-1) / 2)-\beta^{\prime}} \\
& +h^{\left[N_{0} / 2\right]+2+1} \tau^{(\alpha-1) / 2-\left[N_{0} / 2\right]-2} R_{N_{0}} F_{(\alpha-1) / 2} .
\end{aligned}
$$

This completes the computation of $K_{1} . K_{2}$ can be estimated exactly as $K_{1}$. Let us now consider $K_{3}$ :

$$
\begin{aligned}
K_{3} & =\chi_{1}(H) F_{(\alpha-1) / 2}\left[F_{(\alpha-1) / 2}, D A(t)\right] G \chi_{1}(H) \\
& =\chi_{1}(H) F_{(\alpha-1) / 2}(-A)^{-\beta^{\prime}}(-A)^{\beta^{\prime}}\left[F_{(\alpha-1) / 2}, D A(t)\right] G \chi_{1}(H) .
\end{aligned}
$$

Expanding again the commutator $\left[F_{(\alpha-1) / 2}, D A(t)\right]$, we get:

$$
\begin{aligned}
K_{3}= & \sum_{1}^{\left[N_{0} / 2\right]+1} \chi_{1}(H) h^{k} F_{((\alpha-1) / 2)-\beta^{\prime}} B_{k} F_{((\alpha-1) / 2)-k+\beta^{\prime}} \\
& +h^{\left[N_{0} / 2\right]+2} \tau^{(\alpha-1) / 2-\left[N_{0} / 2\right]-2} R_{N_{0}} F_{(\alpha-1) / 2},
\end{aligned}
$$

where as above $B_{k}$ and $R_{N_{0}}$ are uniformly bounded in $h$ and $t$. This completes Step 3.

Step 4. In Step 4, we establish a bound from below on $\left\langle D \Phi_{\alpha}\right\rangle$, which is the key estimate.

We first remark that:

$$
F_{(\alpha-1) / 2} \chi_{1}(H) D A(t) \chi_{1}(H) F_{(\alpha-1) / 2} \geqq C_{0} F_{(\alpha-1) / 2} \chi_{1}^{2}(H) F_{(\alpha-1) / 2} \text { by ii). }
$$

Then we write:

$$
\begin{aligned}
F_{(\alpha-1) / 2} \chi_{1}^{2}(H) F_{(\alpha-1) / 2}= & \chi_{1}(H) F_{\alpha-1} \chi_{1}(H)+\left[F_{(\alpha-1) / 2}, \chi_{1}(H)\right] \chi_{1}(H) F_{(\alpha-1) / 2} \\
& +\chi_{1}(H) F_{(\alpha-1) / 2}\left[\chi_{1}(H), F_{(\alpha-1) / 2}\right]
\end{aligned}
$$

The two commutator terms can be easily estimated as $K_{1}$.

Putting together (6.6), (6.7), (6.9), (6.10), (6.13), (6.14), (6.15), we finally get:

$$
\begin{aligned}
\left\langle D \Phi_{\alpha}\right\rangle \geqq & C_{0}\left\langle\chi_{1}(H) F_{\alpha-1}^{+} \chi_{1}(H)\right\rangle-C h^{N_{0}} \tau^{\alpha-\left(N_{0}+1\right)}\left\|G \psi_{t}\right\|\left\|\psi_{0}\right\| \\
& -C \sum_{k=2}^{N_{0}} \sum_{\ell=0}^{M} h^{k+\ell-1}\left\|F_{\alpha-k-\beta} \psi_{t}\right\|\left\|F_{\beta-\ell} \psi_{t}\right\| \\
& -C \sum_{k=2}^{N_{0}} h^{k+M} \tau^{\beta-(M+1)}\left\|F_{\alpha-k-\beta} \psi_{t}\right\|\left\|\psi_{0}\right\| \\
& -C \sum_{k=1}^{\left[N_{0} / 2\right]+1} h^{k}\left\|F_{((\alpha-1) / 2)-k+\beta^{\prime}} \psi_{t}\right\|\left\|F_{(\alpha-1) / 2-\beta^{\prime}} \psi_{t}\right\| \\
& -C h^{\left[N_{0} / 2\right]+2} \tau^{(\alpha-1) / 2-\left[N_{0} / 2\right]-2}\left\|F_{(\alpha-1) / 2} \psi_{t}\right\|\left\|\psi_{0}\right\| .
\end{aligned}
$$

This completes the proof of the lemma. 
Proof of Theorem 6.1. We will prove the theorem by induction on $\alpha$. Our induction hypothesis will be:

$$
\left(H . N_{0}\right)\left\{\begin{array}{llll}
\left\|F_{\delta}^{+} \psi_{t}\right\|_{L^{2}(d t)}=O\left(h^{n / 2}\right) & \text { for } & \delta<(n-1) / 2, & n \leqq N_{0} . \\
\left\|F_{\delta}^{+} \psi_{t}\right\|_{L^{\infty}(d t)}=O\left(h^{n / 2}\right) & \text { for } \quad \delta<n / 2, & n \leqq N_{0} .
\end{array}\right.
$$

Let us start by proving (H.1).

We take $\alpha<1$, and use Lemma 6.2 with $\beta=\beta^{\prime}=0$. Using that $\alpha-k<-1$ for $k \geqq 2$, we have $\left\|F_{\alpha-k} \psi_{t}\right\|=O\left(\tau^{\alpha-k}\right)\left\|\psi_{0}\right\|$, and also $\left\|F_{((\alpha-1) / 2)-k} \psi_{t}\right\|=O\left(\tau^{((\alpha-1) / 2)-k}\right)\left\|\psi_{0}\right\|$. This gives:

$$
\left\|R_{t}\right\|_{L^{1}(d t)}=O(h)
$$

Using that $\left\langle D \Phi_{\alpha}\right\rangle_{t}=\frac{d}{d t}\left\langle\Phi_{\alpha}\right\rangle_{t}$ and that $\Phi_{\alpha}$ is a negative operator, we get as in [S-S]:

$$
C_{0} \int_{0}^{T}\left\langle F_{\alpha-1}^{+} \psi_{t}, \psi_{t}\right\rangle d t-\left\langle\Phi_{\alpha}(T) \psi_{T}, \psi_{T}\right\rangle \leqq-\left\langle\Phi_{\alpha}(0) \psi_{0}, \psi_{0}\right\rangle+O(h) .
$$

Since $\left\|\Phi_{\alpha}(0) \psi_{0}\right\|=O\left(h^{\infty}\right)$ by $(6.2)$, this proves $(H .1)$.

We assume now that $\left(H . N_{0}\right)$ holds and prove $\left(H . N_{0}+1\right)$. The only thing to do is to estimate $\left\|R_{t}\right\|_{L^{1}(d t)}$.

The terms in $R_{t}$ will be estimated either by Cauchy-Schwarz inequality in $t$ or by $L^{\infty}(d t)-L^{1}(d t)$ estimates. Let us consider successively the different terms: (recall that we take $\alpha<N_{0}+1$ )

a) term $\quad h^{\left[N_{0} / 2\right]+2} \tau^{(\alpha-1) / 2-\left[N_{0} / 2\right]-2}\left\|\psi_{0}\right\|\left\|F_{(\alpha-1) / 2} \psi_{t}\right\|: \quad$ since $\quad \frac{\alpha-1}{2}<N_{0} / 2$, $\tau^{(\alpha-1) / 2-\left[N_{0} / 2\right]-2}$ is in $L^{1}(d t)$. By $\left(H . N_{0}\right),\left\|F_{(\alpha-1) / 2} \psi_{t}\right\|_{L^{\infty}(d t)}=O\left(h^{N_{0} / 2}\right)$, which gives a term with norm in $L^{1}(d t)$ bounded by $C h^{N_{0}+1}$.

b) term $h^{N_{0}} \tau^{\alpha-\left(N_{0}+1\right)}\left\|G \psi_{t}\right\|\left\|\psi_{0}\right\|$ :

$$
h^{N_{0}} \tau^{\alpha-\left(N_{0}+1\right)}\left\|G \psi_{t}\right\|\left\|\psi_{0}\right\|=h^{N_{0}} \tau^{\alpha-\left(N_{0}+1\right)-1 / 2}\left\|\tau^{1 / 2} G \psi_{t}\right\|\left\|\psi_{t}\right\| .
$$

Since $\tau^{\alpha-\left(N_{0}+1\right)-1 / 2} \in L^{2}(d t)$ and $\left\|\tau^{1 / 2} G \psi_{t}\right\| \leqq C\left\|F_{1 / 2} \psi_{t}\right\|$ using that $\left\|F_{1 / 2} \psi_{t}\right\|_{L^{2}(d t)}=$ $O(h)$, we get a term with $L^{1}(d t)$ norm bounded by $C h^{N_{0}+1}$.

c) term $h^{k}\left\|F_{((\alpha-1) / 2)-k+\beta^{\prime}} \psi_{t}\right\|\left\|F_{((\alpha-1) / 2)-\beta^{\prime}} \psi_{t}\right\|$ : we take $\beta^{\prime}=\frac{1}{2}$. Then since $\alpha<N_{0}+1$, we have: $\frac{\alpha-1}{2}-\beta^{\prime}<\frac{N_{0}-1}{2}$, and $\frac{\alpha-1}{2}-k+\beta^{\prime}<\frac{N_{0}-2(k-1)-1}{2}$.

Using the induction hypothesis and Cauchy-Schwarz inequality, we get an $L^{1}(d t)$ norm bounded by $C h^{k} \times h^{N_{0} / 2} h^{N_{0} / 2-k+1}=C h^{N_{0}+1}$.

d) term $h^{k+\ell-1}\left\|F_{\alpha-k-\beta} \psi_{t}\right\|\left\|F_{\beta-\ell} \psi_{t}\right\|$ for $k \geqq 2, \ell \geqq 0$ : since $k \geqq 2$, we have $\alpha-k<N_{0}-1$. Taking $\beta=\frac{\alpha-k}{2}$, we have $\alpha-k-\beta<\frac{N_{0}-1}{2}$ and $\beta-\ell<\frac{N_{0}-1}{2}$.

By the induction hypothesis and Cauchy-Schwarz inequality, we get an $L^{1}(d t)$ norm bounded by $h^{N_{0} / 2} h^{N_{0} / 2} h^{k+\ell-1}=O\left(h^{N_{0}+1}\right)$ since $k \geqq 2$.

e) term $h^{k+M} \tau^{\beta-(M+1)}\left\|F_{\alpha-k-\beta} \psi_{t}\right\|\left\|\psi_{0}\right\|$ : we take $M>\beta$ so that $\tau^{\beta-(M+1)} \in L^{1}(d t)$. Then if $\beta=\frac{\alpha-k}{2}$, one has $\alpha-k-\beta<\frac{N_{0}-1}{2}$, so by induction hypothesis we get: $\left\|F_{\alpha-k-\beta} \psi_{t}\right\|_{L^{\infty}(d t)} \leqq C h^{N_{0} / 2}$. Taking $M$ large enough gives again an $L^{1}(d t)$ norm 
bounded by $C h^{N_{0}+1}$. So we have proved:

$$
\left\|R_{t}\right\|_{L^{1}(d t)}=O\left(h^{N_{0}+1}\right) .
$$

By the same argument as above we get:

$$
C_{0} \int_{0}^{T}\left\langle F_{\alpha-1}^{+} \psi_{t}, \psi_{t}\right\rangle d t-\left\langle\Phi_{\alpha}(T) \psi_{T}, \psi_{T}\right\rangle \leqq-\left\langle\Phi_{\alpha}(0) \psi_{0}, \psi_{0}\right\rangle+O\left(h^{N_{0}+1}\right) .
$$

This implies $\left(H . N_{0}+1\right)$ using again (6.2).

Using now that $F_{\alpha} \geqq \varepsilon \tau^{\alpha} F\left(\frac{A(t)}{\tau} \leqq-\varepsilon\right)$ we get the rest of the theorem.

We will now apply the abstract Theorem 6.1 to Schrödinger operators with smooth potentials.

We consider $P_{h}=-h^{2} \Delta+V(x)$, where $V(x)$ is a potential satisfying the hypotheses in Sect. 2 . We assume that $I$ is an energy interval which is non-trapping for $p=\xi^{2}+V(x)$, so that there exist a modification $G_{1}(x, \xi)$ of the escape function $G$ such that the estimate (2.1) in Proposition 2.2 holds. For simplicity of notations we will still denote by $G$ this new escape function. We take $A(t)=G\left(x, h D_{x}\right)-b t$, where $b$ will be chosen later. We will first state the following Mourre estimate which can be proven as in [Ge1, Proposition 4.1], [Wa, Proposition 3.2].

Proposition 6.3. Let $P_{h}=-h^{2} \Delta+V(x)$, where $V(x)$ is a smooth potential satisfying the hypotheses in Sect. 2 with $V_{s}=0$, such that the energy interval I is non-trapping for $p=\xi^{2}+V(x)$. Then if $\chi_{2}(\lambda)$ is a cutoff function supported in I, there exists some $C_{0}>0$ such that the following Mourre estimate holds for $h$ small enough:

$$
\chi_{2}\left(P_{h}\right)\left[P_{h}, i G\right] \chi_{2}\left(P_{h}\right) \geqq C_{0} h \chi_{2}\left(P_{h}\right)^{2} .
$$

We take then $0<b<C_{0}$. As noted in the beginning of the section, we will have to replace $P_{h}$ by $P_{h, 1}=\chi_{0}\left(P_{h}\right) P_{h}$, where $\chi_{0}(\lambda)$ is a large cutoff function equal to 1 near $I$.

Finally we take a cutoff function $\chi_{\text {out }}(x, \xi)$ supported in

$$
\left\{(x, \xi) \in T^{*}\left(\mathbb{R}^{n}\right) \mid p(x, \xi) \in I, G(x, \xi) \geqq C_{1}\right\} \quad \text { for } \quad C_{1}>0 .
$$

We denote by $\chi_{\text {out }}$ the operator $\chi_{\text {out }}\left(x, h D_{x}\right)$. We start by proving a proposition which shows that we can apply Theorem 6.1 (recall that $P_{h, 1}=\chi_{0}\left(P_{h}\right) P_{h}$ for some large cutoff function $\chi_{0}$ ):

Proposition 6.4. i) $\left(\widetilde{\operatorname{ad}}_{A(t)}\right)^{k}\left(P_{h, 1}\right)$ is bounded uniformly in $h$.

ii) $\chi_{1}\left(P_{h}\right)\left[P_{h, 1}, i A\right] \chi_{1}\left(P_{h}\right) \geqq\left(C_{0}-\varepsilon\right) h \chi_{1}^{2}\left(P_{h}\right)$ for any $\varepsilon>0$, for $h \leqq h(\varepsilon)$.

iii) $(-A(0))^{\alpha} F(A(0) \leqq(b-\varepsilon)) \chi_{1}\left(P_{h}\right) \chi_{\text {out }}$ is bounded with norm $O\left(h^{\infty}\right)$.

Proof. Let us first prove i). As in Sect. 5, we use the following formula to define $P_{h, 1}=\chi_{0}\left(P_{h}\right) P_{h}$ : if $K(x)=x \chi_{0}(x)$ we denote by $\tilde{K}(z)$ an almost analytic extension of $\tilde{K}(x)$ which can be taken with compact support. Then we have:

$$
P_{h, 1}=\frac{i}{2 \pi} \int_{\mathbb{C}} \frac{\partial \tilde{K}}{\partial \bar{z}}(z)\left(z-P_{h}\right)^{-1} d z \wedge d \bar{z}
$$


Then:

$$
\begin{aligned}
& h^{-1} {\left[P_{h, 1} A(t)\right] } \\
&=h^{-1}\left[P_{h 1}, G\right]=\frac{i}{2 \pi} \int_{\mathbb{C}} \frac{\partial \tilde{K}}{\partial \bar{z}}(z)\left(z-P_{h}\right)^{-1} h^{-1}\left[P_{h}, G\right]\left(z-P_{h}\right)^{-1} d z \wedge d \bar{z} \\
&=\frac{i}{2 \pi} \int_{\mathbb{C}} \frac{\partial \tilde{K}}{\partial \bar{z}}(z)\left(z-P_{h}\right)^{-1}\left(P_{h}+i\right) h^{-1}\left(P_{h}+i\right)^{-1}\left[P_{h}, G\right]\left(z-P_{h}\right)^{-1} d z \wedge d \bar{z} .
\end{aligned}
$$

Since $h^{-1}\left(P_{h}+i\right)^{-1}\left[P_{h}, G\right]$ is bounded and $\left\|\left(z-P_{h}\right)^{-1}\right\|_{\mathscr{S}\left(L^{2}, H^{2}\right)}=O\left(|\operatorname{Im} z|^{-1}\right)$ we get that $h^{-1}\left[P_{h, 1}, A(t)\right]$ is bounded uniformly in $h$.

Using (6.19) to write $h^{-2}\left[\left[P_{h, 1}, A(t)\right] A(t)\right]$ gives that $\left(\tilde{\mathrm{ad}}_{A(t)}\right)^{2}\left(P_{h, 1}\right)$ is bounded uniformly in $h$. The general case follows by induction, using that $\left(\tilde{a d}_{G}\right)^{k}\left(P_{h}\right)$ is bounded uniformly in $h$ from $H^{2}(X)$ into $L^{2}(X)$.

Let us now prove ii).

We have:

$$
\chi_{1}\left(P_{h}\right)\left[P_{h, 1}, i A\right] \chi_{1}\left(P_{h}\right)=\chi_{1}\left(P_{h}\right) \chi_{0}\left(P_{h}\right)\left[P_{h}, i G\right] \chi_{1}\left(P_{h}\right)+\chi_{1}\left(P_{h}\right)\left[\chi_{0}\left(P_{h}\right), i G\right] P_{h} \chi_{1}\left(P_{h}\right) .
$$

By (6.18), the first term is bigger than $C_{0} h_{\chi 1}\left(P_{h}\right)^{2}$. Let us consider the second term.

We first take a function $F_{1}(\lambda)$, constant at $\pm \infty$ such that if $P_{h}^{\prime}=F_{1}\left(P_{h}\right)$, one has: $\chi_{0}\left(P_{h}\right)=\chi_{0}\left(P_{h}^{\prime}\right)$. Let us check that $\left[G, P_{h}^{\prime}\right]=O(h)$ and $\left[\left[G, P_{h}^{\prime}\right], P_{h}^{\prime}\right]=O\left(h^{2}\right)$. The fact that $\left[G, P_{h}^{\prime}\right]=O(h)$ follows from i), by noticing that one can take an

almost analytic extension $\tilde{F}_{1}(z)$ of $F_{1}(x)$ such that $\frac{\partial \widetilde{F}_{1}}{\partial \bar{z}}$ has compact support.
Since $P_{h}^{\prime}$ commutes with $\left(z-P_{h}\right)^{-1}$, we have:

$$
\left[\left[G, P_{h}^{\prime}\right], P_{h}^{\prime}\right]=\frac{i}{2 \pi} \int_{\mathbb{C}} \frac{\partial \tilde{F}_{1}}{\partial \bar{z}}\left(z-P_{h}\right)^{-1}\left[\left[G, P_{h}\right], P_{h}^{\prime}\right]\left(z-P_{h}\right)^{-1} d z \wedge d \bar{z} .
$$

Now:

$$
\left[\left[G, P_{h}\right], P_{h}^{\prime}\right]=\frac{-i}{2 \pi} \int_{\mathbb{C}} \frac{\partial \tilde{F}_{1}}{\partial \bar{z}}\left(z-P_{h}\right)^{-1}\left[P_{h},\left[G, P_{h}\right]\right]\left(z-P_{h}\right)^{-1} d z \wedge d \bar{z} .
$$

Since $\left(P_{h}+i\right)^{-1}\left[P_{h},\left[G, P_{h}\right]\right]\left(P_{h}+i\right)^{-1}$ has a norm $O\left(h^{2}\right),\left[\left[G, P_{h}\right], P_{h}^{\prime}\right]$ and $\left[\left[G, P_{h}^{\prime}\right], P_{h}^{\prime}\right]$ have also norms $O\left(h^{2}\right)$. Then we can apply Proposition 5.1 to expand $\left[\chi_{0}\left(P_{h}\right), i G\right]$ to order 1 . The first term is $\chi_{0}^{\prime}\left(P_{h}\right)\left[P_{h}, i G\right]$ which gives 0 by left multiplication with $\chi_{1}\left(P_{h}\right)$. The remainder term is $O\left(h^{2}\right)$. So this gives ii), for $h$ small enough.

Let us now prove iii). We write

$$
G^{\alpha} F(G \leqq b-\varepsilon) \chi_{1}\left(P_{h}\right) \chi_{\text {out }}=G^{-[\alpha]+\alpha-1} F(G \leqq b-\varepsilon) G^{[\alpha]+1} \chi_{1}\left(P_{h}\right) \chi_{\text {out }} .
$$

Using the functional calculus of Helffer-Robert [He-Ro] adapted to non-conformal metrics in [Ge1], we get that for any $N$ :

$$
\chi_{1}\left(P_{h}\right)=\chi_{p s}\left(x, h D_{x}\right)+R_{N}(h)
$$

where $\chi_{p s}\left(x, h D_{x}\right)$ is a pseudodifferential operator with total symbol equal to 1 near $\operatorname{supp} \chi_{\text {out }}(x, \xi)$ and $R_{N}(h)$ has an operator norm $O\left(h^{N}\right)$ between any weighted Sobolev spaces $H_{\langle x\rangle^{\boldsymbol{k}}}^{s}$. 
This gives that modulo error terms of norm $O\left(h^{N}\right), G^{[\alpha]+1} \chi_{1}\left(P_{h}\right) \chi_{\text {out }}$ is a pseudodifferential operator with total symbol supported in $\left\{(x, \xi) \mid G(x, \xi) \geqq C_{1}\right\}$. Then by applying Proposition 5.2, we get that $F(G \leqq b-\varepsilon) G^{[\alpha]+1} \chi_{1}\left(P_{h}\right) \chi_{\text {out }}$ has a norm $O\left(h^{\infty}\right)$. This completes the proof of proposition.

We are now able to apply Theorem 6.1 to $M$. So we get:

Corollary 6.5. Let $P_{h}, b$ and $G$ be as Proposition 6.4. Then one has:

$$
\left\|F\left(\frac{G\left(x, h D_{x}\right)}{t}-b \leqq \varepsilon\right) e^{-i t P_{h} / h} \chi_{1}\left(P_{h}\right) \chi_{\text {out }} u\right\| \leqq C_{N} h^{N}\langle t\rangle^{-N} \quad \forall N \in \mathbb{N} \text {. }
$$

\section{Minimal Velocity Bounds}

In this section we prove minimal velocity bounds in the semiclassical limit by adapting the arguments of $[\mathrm{S}-\mathrm{S}]$. We assume that $P_{h}=-h^{2} \Delta_{x}+V(x)$ is a Schrödinger operator with potential $V$ satisfying the hypotheses of Sect. 2 with $V_{s}=0$ and the Mourre estimate (6.18).

We denote by $G_{\alpha}$ the propagation observable

$$
G_{\alpha}=-\left(b t^{2}-x^{2}\right)^{\alpha / 2} F\left(\frac{x^{2}}{\tau^{2}} \leqq v^{2}\right) .
$$

We take $v^{2}<b<C_{0}$, where $C_{0}$ is the constant in (6.18). We denote by $A_{0}$ the generator of dilations $\frac{1}{2} h\left(x \cdot D_{x}+D_{x} \cdot x\right)$.

We first prove a lemma which extends [S-S, Lemma 3.2]:

Lemma 7.1. $\exists T_{0}$ such that one has for $t \geqq T_{0}$ :

$$
D G_{x} \geqq \delta t\left(-G_{\alpha-2}\right)+R_{0, t}+O\left(h^{\infty}\langle t\rangle^{-\infty}\right),
$$

where $\left\langle\boldsymbol{R}_{0, t}\right\rangle$ satisfies:

$$
\left|\left\langle R_{0, t}\right\rangle\right| \leqq C h \tau^{\alpha-2}\left\|G_{0} \psi_{t}\right\|^{2}
$$

Proof. We have:

$$
i h^{-1}\left[P_{h}, G_{\alpha}\right]=2 G_{\alpha}^{\prime} x \cdot h D_{x}+\frac{i h}{2} \Delta G_{\alpha}
$$

where $G_{\alpha}^{\prime}=\frac{\partial G_{\alpha}}{\partial|x|^{2}}$
So:

$$
i h^{-1}\left[P_{h}, G_{\alpha}\right]=2 G_{\alpha}^{\prime} A_{0}+\frac{i h}{2} \Delta G_{\alpha}-2 h G_{\alpha}^{\prime}
$$

Since $G_{\alpha}^{\prime} \geqq 0$, we have:

$$
G_{\alpha}^{\prime}=\left(F_{(\alpha / 2)-1}\right)^{2}
$$

where we will denote by $F_{\delta}$ an operator of the form $t^{\delta} \times K_{\delta}\left(\frac{\langle x\rangle}{\tau}, t\right)$, where $K_{\delta}(s, t)$ is a bounded function supported in $\{s \in \mathbb{R} \mid s \leqq v\}$. 
With this notation, we get:

$$
i h^{-1}\left[P_{h}, G_{\alpha}\right]=2 F_{(\alpha / 2)-1} A_{0} F_{(\alpha / 2)-1}+R_{0, \alpha},
$$

where $R_{0, \alpha}$ is a multiplication operator of the form $h \tau^{\alpha-2} K_{0}\left(\frac{\langle x\rangle}{\tau}\right)$. By Hypothesis (H.5) and Proposition 2.2, we know that $G\left(x, h D_{x}\right)=A_{0}+R_{0}$, where $R_{0}$ is a bounded operator, and we get:

$$
i h^{-1}\left[P_{h}, G_{\alpha}\right]=2 F_{(\alpha / 2)-1} G F_{(\alpha / 2)-1}+R_{0, \alpha}+2 F_{(\alpha / 2)-1} R_{0} F_{(\alpha / 2)-1} .
$$

Now we use that:

which gives:

$$
G \geqq a t F\left(\frac{G}{t} \geqq a\right)+G F(G \leqq 0)
$$

$$
i h^{-1}\left[P_{h}, G_{\alpha}\right] \geqq 2 a t G_{\alpha}^{\prime}+R_{0, \alpha}+2 F_{(\alpha / 2)-1} R_{0} F_{(\alpha / 2)-1}+R_{1, \alpha}+R_{2, \alpha},
$$

where:

$$
\begin{aligned}
& R_{1, \alpha}=2 a t F_{(\alpha / 2)-1} F\left(\frac{G}{\tau} \leqq a\right) F_{(\alpha / 2)-1}, \\
& R_{2, \alpha}=2 F_{(\alpha / 2)-1} G F(G \leqq 0) F_{(\alpha / 2)-1} .
\end{aligned}
$$

We can rewrite $R_{2, \alpha}$ as:

$$
\begin{aligned}
R_{2, \alpha} & =2 F_{(\alpha / 2)-1} G F(G \leqq-\delta \tau) F_{(\alpha / 2)-1}+2 F_{(\alpha / 2)-1} G F(-\delta \tau \leqq G \leqq 0) F_{(\alpha / 2)-1} \\
& =\tilde{R}_{2, \alpha}+\tilde{R}_{3, \alpha} .
\end{aligned}
$$

We have:

$$
\begin{aligned}
\left|\left\langle\tilde{R}_{3, \alpha}\right\rangle\right| & =\left|\left(F_{(\alpha / 2)-1} G F(-\delta \tau \leqq G \leqq 0) F_{(\alpha / 2)-1} \psi_{t}, \psi_{t}\right)\right| \\
& \leqq 2 \delta \tau\left\|F_{(\alpha / 2)-1} u_{t}\right\|^{2} .
\end{aligned}
$$

Using (7.4) and the fact that $R_{0}$ is bounded, we get:

$$
i h^{-1}\left[P_{h}, G_{\alpha}\right] \geqq 2(\alpha-2 \delta) t F_{\alpha-2}+R_{0, \alpha}+R_{1, \alpha}+\tilde{R}_{2, \alpha} \text {, for } t \geqq T_{0} .
$$

In the next step we will estimate the terms $R_{1, \alpha}$ and $\tilde{R}_{2, \alpha}$. Let us begin with $R_{1, \alpha}$. We have:

$$
F_{(\alpha / 2)-1} F\left(\frac{G}{\tau} \leqq a\right) F_{(\alpha / 2)-1}=F_{\alpha-2} F\left(\frac{G}{\tau} \leqq a\right)+F_{(\alpha / 2)-1}\left[F\left(\frac{G}{\tau} \leqq a\right), F_{(\alpha / 2)-1}\right] .
$$

It is straightforward using pseudodifferential calculus and hypothesis $(H .5)$ to check that $\left(\tilde{\operatorname{ad}}_{G}\right)^{k}\left(K_{\alpha}\left(\frac{\langle x\rangle}{\tau}, t\right)\right)$ is bounded uniformly in $h$ and $t$.

Applying then the commutator expansion, we get:

$$
\begin{aligned}
{\left[F\left(\frac{G}{\tau} \leqq a\right), F_{(\alpha / 2)-1}\right]=} & t^{(\alpha / 2)-1} \sum_{1}^{N-1}(-1)^{k} \frac{h^{k}}{\tau^{k}}\left(\widetilde{a d}_{G}\right)^{k}\left(K_{\alpha}\right) F^{(k)}\left(\frac{G}{\tau} \leqq a\right) \\
& +t^{(\alpha / 2)-1} O\left(\frac{h^{N}}{\tau^{N}}\right) .
\end{aligned}
$$


From this we obtain:

$$
\begin{aligned}
\left\langle R_{1, \alpha}\right\rangle= & \left(R_{1, \alpha} \psi_{t}, \psi_{t}\right)=\left(F\left(\frac{G}{\tau} \leqq a\right) \psi_{t}, F_{\alpha-2} \psi_{t}\right) \\
& +\sum_{1}^{N} O\left(\tau^{(\alpha / 2)-1-k}\right) h^{k}\left\|F\left(\frac{G}{\tau} \leqq a\right) \psi_{t}\right\|\left\|F_{(\alpha / 2)-1} \psi_{t}\right\| \\
& +O\left(h^{N}\right) \tau^{(\alpha / 2)-1-N}\left\|\psi_{0}\right\|^{2}
\end{aligned}
$$

Let us now estimate $\tilde{R}_{2, \alpha}$. We have:

$$
\begin{aligned}
F_{(\alpha / 2)-1} G F(G \leqq-\delta \tau) F_{(\alpha / 2)-1}= & F_{\alpha-2} G F(G \leqq-\delta \tau) \\
& +F_{(\alpha / 2)-1}\left[G F(G \leqq-\delta \tau), F_{(\alpha / 2)-1}\right]
\end{aligned}
$$

The second term in the r.h.s. of (7.7) is equal to:

$$
t^{\alpha-2} K_{\alpha}\left[G F(G \leqq-\delta \tau), K_{\alpha}\right]
$$

Expanding the commutator gives:

$$
\left[G F(G \leqq-\delta \tau), K_{\alpha}\right]=\sum_{1}^{N} h^{k}(-1)^{k} \tau^{1-k}\left(\tilde{a d}_{G}\right)^{k}\left(K_{\alpha}\right) F_{1}^{(k)}\left(\frac{G}{\tau} \leqq-\delta\right)+O\left(\frac{h^{N}}{\tau^{N-1}}\right)
$$
Here we write $G F(G \leqq-\delta \tau)=\tau F_{1}\left(\frac{G}{\tau}\right)$, where $F_{1}(s)=s F(s \leqq-\delta)$. From this we
get:

$$
\begin{aligned}
\left\langle\tilde{R}_{2, \alpha}\right\rangle= & t^{\alpha-2}\left\langle G F(G \leqq-\delta \tau) \psi_{t}, K_{\alpha} \psi_{t}\right\rangle \\
& +\sum_{1}^{N} h^{k} O\left(t^{\alpha-1-k}\right)\left\|F_{1}^{(k)}\left(\frac{G}{\tau} \leqq-\delta\right) \psi_{t}\right\|\left\|K_{\alpha} \psi_{t}\right\| \\
& +t^{\alpha-1-N} h^{N}\left\|\psi_{0}\right\|^{2} .
\end{aligned}
$$

Using the estimates of Theorem 6.1 with $A(t)=G$, we get finally that:

$$
\left|\left\langle R_{1, \alpha}\right\rangle\right|+\left|\left\langle\tilde{R}_{2, \alpha}\right\rangle\right| \leqq C_{N} h^{N}\langle t\rangle^{-N} \quad \forall N .
$$

To complete the proof of the lemma, we just have to compute $\frac{d G_{\alpha}}{d t}$. As in $[\mathrm{S}-\mathrm{S}]$, we have:

$$
\begin{aligned}
\frac{d G_{\alpha}}{d t} & =\alpha b t G_{\alpha-2}-2\left(b t^{2}-x^{2}\right)^{\alpha / 2} \frac{x^{2}}{\tau^{3}}\left(-F^{\prime}\right)\left(\frac{x^{2}}{\tau^{2}} \leqq v^{2}\right) \\
& \geqq \alpha b t G_{\alpha-2}+2 \frac{v^{2}}{\tau} .
\end{aligned}
$$

Since also $G_{\alpha}^{\prime}=\frac{\alpha}{2}\left(-G_{\alpha-2}\right)-\frac{\left(b t^{2}-|x|^{2}\right)^{\alpha / 2}}{\tau^{2}} F^{\prime}$, we get for $t \geqq T_{0}$ :

$$
D G_{\alpha} \geqq \delta t\left(-G_{\alpha-2}\right)+\frac{\delta}{\tau} \frac{\left(b t^{2}-|x|^{2}\right)^{\alpha / 2}}{\tau^{2}}\left(-F^{\prime}\right)+R_{0, \alpha}+O\left(h^{N}\langle t\rangle^{-N}\right),
$$

which proves the lemma. 
We now prove the analog of [S-S, Theorem 3.1]. We use the notations of the end of Sect. 6.

We ask moreover that the cutoff function $\chi_{\text {out }}(x, \xi)$ is supported in

$$
\left\{x \in \mathbb{R}^{n}|| x \mid \geqq v\left(T_{0}+1\right)+\varepsilon_{0}\right\},
$$

where $T_{0}$ is the time in Lemma 7.1.

Theorem 7.2. Let $\chi_{1}(\lambda), \chi_{\text {out }}\left(x, h D_{x}\right)$ be as in Proposition 6.4. Then for $t \geqq T_{0}$, one has:

$$
\left\|\chi(|x| \leqq v(t+1)) e^{-i t P_{h} / h} \chi_{1}\left(P_{h}\right) \chi_{\text {out }} u\right\| \leqq C_{N} h^{N}\langle t\rangle^{-N}\|u\| \quad \forall N \in \mathbb{N} .
$$

Proof. Similarly to Theorem 6.1, the proof is done by considering the observable $G_{\alpha}$ and by induction on $\alpha$. Lemma 7.1 and negativity of the observable $G_{\alpha}$ gives:

$$
\begin{aligned}
- & \left\langle G_{\alpha}(T)\right\rangle_{T}+\delta \int_{T_{0}}^{T} t\left\langle-G_{\alpha-2} \psi_{t}, \psi_{t}\right\rangle d t \\
& \leqq-\left\langle G_{\alpha}\left(T_{0}\right)\right\rangle_{T_{0}}+C_{N} h^{N}+C h \int_{T_{0}}^{T}\langle t\rangle^{\alpha-2}\left\|G_{0} \psi_{t}\right\|^{2} d t .
\end{aligned}
$$

By the pseudodifferential calculus, we have

$$
\left\|\chi\left(|x| \leqq v\left(T_{0}+1\right)\right) \chi_{1}\left(P_{h}\right) \chi_{\text {out }}\right\|=O\left(h^{\infty}\right)
$$

so

$$
\left\langle G_{\alpha}\left(T_{0}\right)\right\rangle_{T_{0}}=O\left(h^{\infty}\right)
$$

For $\alpha<1,(7.9)$ yields:

$$
\begin{aligned}
\int_{T_{0}}^{+\infty}\langle t\rangle^{\delta}\left\|G_{0} \psi_{t}\right\|^{2} d t \leqq C h & \forall \delta<0 \\
\forall t \geqq T_{0}:\langle t\rangle^{\delta}\left\|G_{0} \psi_{t}\right\|^{2} \leqq C h & \forall \delta<1 .
\end{aligned}
$$

The last estimate follows from:

$$
G_{\alpha} \geqq C\langle t\rangle^{\alpha} G_{0} .
$$

Let us check by induction on $N$ that one has:

$$
\begin{aligned}
& \int_{T_{0}}^{+\infty}\langle t\rangle^{\delta}\left\|G_{0} \psi_{t}\right\|^{2} d t \leqq C_{N} h^{N} \forall \delta<N-1, \\
& \forall t \geqq T_{0} \quad\langle t\rangle^{\delta}\left\|G_{0} \psi_{t}\right\|^{2} \leqq C_{N} h^{N} \quad \forall \delta<N .
\end{aligned}
$$

Assume that (7.10), (7.11) hold for $N=N_{0}$. Using (7.9) for $\alpha<N_{0}+1$, and (7.10) for $\delta<N_{0}-1$ to estimate the r.h.s. of (7.9) gives immediately (7.10), (7.11) for $N=N_{0}+1$.

This completes the proof of the theorem.

For use in Sect. 3, we will now consider the case of an Hamiltonian satisfying the hypotheses $(H)$ of Sect. 2. From hypothesis (H.6), there are no trapped trajectories on $p^{-1}(I)$ near infinity, but we assume that there may exist trapped trajectories near the origin. In other words the semiclassical Mourre estimate (6.18) does not hold. By hypothesis $(H .7)$, there exists a $C_{0}^{\infty}$ function $W$ such that if $p_{1}=p+W, I$ 
is non-trapping for $p_{1}$. We put $P_{h, 1}=P_{h}+W-v_{s}$. By Proposition 2.2, one can modify the escape function $G$ in a compact set of $T^{*}\left(\mathbb{R}^{n}\right)$ such that $H_{p_{1}} G \geqq c_{0}$ on $p_{1}^{-1}(I)$. We will still denote by $G$ this new modified escape function.

Let us denote by $\chi_{\text {out }}(x, \xi) \in C_{0}^{\infty}\left(T^{*} \mathbb{R}^{n}\right)$ a cutoff function supported in

$$
\left\{(x, \xi)\left|G(x, \xi) \geqq C_{1}+1,\right| x \mid \geqq R_{1}\right\},
$$

where $R_{1}$ is large enough such that supp $V_{s} \subset\left\{|x| \leqq R_{1}-1\right\}$ and:

$$
C_{1}=\sup _{x \in \operatorname{supp} W \cup \operatorname{supp} V s, p_{1}(x, \xi) \in I} G(x, \xi) .
$$

The following remark will be used in the proof of Theorem 7.3 below:

Remark 5. Since $H_{p_{1}} G \geqq c_{0}$ on $p_{1}^{-1}(I)$, we get that:

$\forall(x, \xi) \in \operatorname{supp} \chi_{\text {out }} \bigcap_{1}^{-1}(I), \forall t \geqq 0$, one has: $\pi_{x}\left(\exp t H_{p_{1}}(x, \xi)\right) \notin \operatorname{supp} W \cup \operatorname{supp} V_{s}$.

Then we have the following result:

Theorem 7.3. Let $\chi(\lambda)$ be a cutoff function supported in $p^{-1}(I)$. Then:

$$
\chi\left(P_{h}\right) e^{i t P_{h} / h} \chi_{\text {out }}\left(x, h D_{x}\right)=\chi\left(P_{h, 1}\right) e^{-i t P_{h, 1} / h} \chi_{\text {out }}\left(x, h D_{x}\right)+R_{\infty}(h)
$$

where:

$$
\left\|R_{\infty}(h)\right\|_{\mathscr{L}\left(L^{2}(X)\right)}=O\left(h^{\infty}\right) \text { uniformly in } t \in \mathbb{R}^{+} .
$$

Proof. We use the following standard formula:

$$
e^{-i t P_{h} / h}=e^{-i t P_{h, 1} / h}-(i h)^{-1} \int_{0}^{t} e^{-i(t-s) P_{h} / h}\left(W-V_{s}\right) e^{-i s P_{h, 1} / h} d s
$$

Let us take another cutoff function $\chi_{1}(\lambda)$ supported in $p^{-1}(I)$ with $\chi(\lambda) \chi_{1}(\lambda)=\chi(\lambda)$. Using the functional calculus ([He-Ro], [Ge1]), Proposition 5.4, and the fact that $\chi_{\text {out }}$ is supported away from the support of $V_{s}$, we get that:

$$
\chi_{1}\left(P_{h}\right) \chi_{\text {out }}=\tilde{\chi}_{\text {out }}\left(x, h D_{x}, h\right)+R_{\infty}(h),
$$

where $\tilde{\chi}_{\text {out }}\left(x, h D_{x}, h\right)$ is a pseudodifferential operator with total symbol supported in $\operatorname{supp} \chi_{\text {out }} \cap p^{-1}(I)$, and $R_{\infty}(h)$ has an operator norm $O\left(h^{\infty}\right)$. From this we get:

$$
\begin{aligned}
\chi\left(P_{h}\right) e^{-i t P_{h} / h} \chi_{\text {out }} & =\chi\left(P_{h}\right) e^{-i t P_{h} / h} \chi_{1}\left(P_{h}\right) \chi_{\text {out }} \\
& =\chi\left(P_{h}\right) e^{-i t P_{h} / h} \tilde{\chi}_{\text {out }}\left(x, h D_{x}, h\right)+R_{\infty}(t, h),
\end{aligned}
$$

where $R_{\infty}(t, h)$ has an operator norm $O\left(h^{\infty}\right)$ uniformly in $h$.

By the same argument, using that on $\operatorname{supp} \tilde{\chi}_{\text {out }}(x, \xi, h) p$ is equal to $p_{1}$, we have:

$$
\chi\left(P_{h}\right) e^{-i t P_{h} / h} \tilde{\chi}_{\text {out }}\left(x, h D_{x}, h\right)=\chi\left(P_{h}\right) e^{-i t P_{h} / h} \chi_{2}\left(P_{h, 1}\right) \tilde{\chi}_{\text {out }}\left(x, h D_{x}, h\right)+R_{\infty}(t, h) .
$$

Here $\chi_{2}(\lambda)$ is another function equal to 1 on $I$. Using (7.12) we get:

$$
\begin{aligned}
\chi\left(P_{h}\right) e^{-i t P_{h} / h} \chi_{\text {out }}= & \chi\left(P_{h}\right) e^{-i t P_{h, 1} / h} \chi_{2}\left(P_{h, 1}\right) \tilde{\chi}_{\text {out }} \\
& -(i h)^{-1} \int_{0}^{t} e^{-i(t-s) P_{h} / h} \chi\left(P_{h}\right)\left(W-V_{s}\right) e^{-i s P_{h, 1} / h} \\
& \cdot \chi_{2}\left(P_{h, 1}\right) \tilde{\chi}_{\text {out }} d s+O\left(h^{\infty}\right) .
\end{aligned}
$$


Let us consider now $\chi\left(P_{h}\right)\left(W-V_{s}\right) e^{-i s P_{h, 1} / h} \chi_{2}\left(P_{h, 1}\right) \tilde{\chi}_{\text {out }}$.

We first remark that by Egorov's theorem (see [Ro]) and using the Remark 5, we get:

$$
\left\|\chi\left(P_{h}\right)\left(W-V_{s}\right) e^{-i s P_{h, 1} / h} \chi_{2}\left(P_{h, 1}\right) \tilde{\chi}_{\text {out }}\right\|=O_{s}\left(h^{\infty}\right) .
$$

So we only have to consider what happens for $s \geqq s_{0}, s_{0} \gg 1$. We have:

$$
\begin{aligned}
\chi\left(P_{h}\right) & \left(W-V_{s}\right) e^{-i s P_{h, 1} / h} \chi_{2}\left(P_{h, 1}\right) \tilde{\chi}_{\text {out }} \\
= & \chi\left(P_{h}\right)\left(W-V_{s}\right) F(G \leqq b\langle s\rangle) \chi_{2}\left(P_{h, 1}\right) e^{-i s P_{h, 1} / h} \tilde{\chi}_{\text {out }} \\
& +\chi\left(P_{h}\right)\left(W-V_{s}\right) \chi_{2}\left(P_{h, 1}\right) F(G \geqq b\langle s\rangle) e^{-i s P_{h, 1} / h} \tilde{\chi}_{\text {out }} \\
= & I_{1}(s)+I_{2}(s) .
\end{aligned}
$$

Using Corollary 6.5 applied to $P_{h, 1}$ and the fact that $V_{s}$ is $P_{h}$-bounded, we get that:

$$
\left\|I_{1}(s)\right\|=\frac{O\left(h^{\infty}\right)}{\langle s\rangle^{\infty}} \text { for } s \geqq s_{0} .
$$

Let us consider now $I_{2}(s)$. We write:

$$
\begin{aligned}
& \langle s\rangle^{N} \chi\left(P_{h}\right)\left(W-V_{s}\right) \chi_{2}\left(P_{h, 1}\right) F(G \geqq b\langle s\rangle) \\
& \quad=\chi\left(P_{h}\right)\left(W-V_{s}\right) \chi_{2}\left(P_{h, 1}\right)(G+i)^{N} F_{1}(G \geqq b\langle s\rangle)\langle s\rangle^{N}(G+i)^{-N} F(G \geqq b\langle s\rangle) .
\end{aligned}
$$

Here $F_{1}(\lambda)$ is a cutoff function such that $F_{1} F=F$. One first notices that:

$$
\left\|\langle s\rangle^{N}(G+i)^{-N} F(G \geqq b\langle s\rangle)\right\| \leqq C_{N} .
$$

Then we use that $V_{s}$ and $W$ are compactly supported multiplicative potentials. We take a cutoff function $\chi_{0}(x) \in C_{0}^{\infty}\left(\mathbb{R}^{n}\right)$ such that $\left(W-V_{s}\right)=\left(W-V_{s}\right) \chi_{0}$. So we have:

$$
\begin{aligned}
& \chi\left(P_{h}\right)\left(W-V_{s}\right) \chi_{2}\left(P_{h, 1}\right)(G+i)^{N} F_{1}(G \geqq b\langle s\rangle) \\
& \quad=\chi\left(P_{h}\right)\left(W-V_{s}\right) \chi_{0}(x) \chi_{2}\left(P_{h, 1}\right)(G+i)^{N} F_{1}(G \geqq b\langle s\rangle) .
\end{aligned}
$$

Then $\chi\left(P_{h}\right)\left(W-V_{s}\right)$ is a bounded operator and $\chi_{0}(x) \chi_{2}\left(P_{h, 1}\right)(G+i)^{N}$ is (modulo a term of norm $\left.O\left(h^{\infty}\right)\right)$, a pseudodifferential operator with symbol supported in a region where $|G(x, \xi)| \leqq C_{1}+\varepsilon_{0}$. Let $\chi_{1}(\lambda)$ be a cutoff function equal to 1 near $\left[-C_{1}-\varepsilon_{0}, C_{1}+\varepsilon_{0}\right]$.

Then using Proposition 5.2, we get that:

$$
\chi_{0}(x) \chi_{2}\left(P_{h, 1}\right)(G+i)^{N}\left(1-\chi_{1}(G)\right)=O\left(h^{\infty}\right) .
$$

So:

$$
\begin{aligned}
& \chi\left(P_{h}\right)\left(W-V_{s}\right) \chi_{2}\left(P_{h, 1}\right)(G+i)^{N} F_{1}(G \geqq b\langle s\rangle) \\
& \quad=\chi\left(P_{h}\right)\left(W-V_{s}\right) \chi_{0}(x) \chi_{2}\left(P_{h, 1}\right)(G+i)^{N} \chi_{1}(G) F_{1}(G \geqq b\langle s\rangle)+O\left(h^{\infty}\right)=O\left(h^{\infty}\right)
\end{aligned}
$$

for $s \geqq s_{0}$ with $b\left\langle s_{0}\right\rangle \geqq 2\left(C_{1}+\varepsilon_{0}\right)$.

Using (7.19) we get:

$$
\left\|I_{2}(s)\right\|=\frac{O\left(h^{\infty}\right)}{\langle s\rangle^{\infty}} \text { for } \quad s \geqq s_{0} .
$$




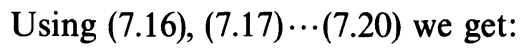

$$
\chi\left(P_{h}\right) e^{-i t P_{h} / h} \chi_{\text {out }}=\chi\left(P_{h}\right) e^{-i t P_{h, 1} / h} \chi_{2}\left(P_{h, 1}\right) \tilde{\chi}_{\text {out }}+O\left(h^{\infty}\right) .
$$

Using again Remark 5 and Corollary 6.5, we get:

$$
\chi\left(P_{h}\right) e^{-i t P_{h, 1} / h} \chi_{2}\left(P_{h, 1}\right) \tilde{\chi}_{\text {out }}=\chi\left(P_{h}\right) F\left(|x| \geqq C+\varepsilon_{0}\right) e^{-i t P_{h, 1} / h} \chi_{2}\left(P_{h, 1}\right) \tilde{\chi}_{\text {out }}+O\left(h^{\infty}\right) \text {. }
$$

Here $C$ is such that $\operatorname{supp}\left(W+V_{s}\right) \subset\{|x| \leqq C\}$ and $\varepsilon_{0}$ is small enough. Then by Proposition 5.4 we have:

$$
\chi\left(P_{h}\right) F\left(|x| \geqq C+\varepsilon_{0}\right)=\chi\left(P_{h, 1}\right) F\left(|x| \geqq C+\varepsilon_{0}\right)+O\left(h^{\infty}\right) .
$$

So:

$$
\begin{aligned}
\chi\left(P_{h}\right) e^{-i t P_{h} / h} \chi_{\text {out }} & =\chi\left(P_{h, 1}\right) F\left(|x| \geqq C+\varepsilon_{0}\right) e^{-i t P_{h, 1} / h} \chi_{2}\left(P_{h, 1}\right) \tilde{\chi}_{\text {out }}+O\left(h^{\infty}\right) \\
& =e^{-i t P_{h, 1} / h} \chi\left(P_{h, 1}\right) \tilde{\chi}_{\text {out }}+O\left(h^{\infty}\right) .
\end{aligned}
$$

(Here and above the remainders $O\left(h^{\infty}\right)$ are uniform in $t \in \mathbb{R}^{+}$). But again by Proposition 5.4, $\tilde{\chi}_{\text {out }}=\chi_{1}\left(P_{h}\right) \chi_{\text {out }}+O\left(h^{\infty}\right)($ see $(7.13))=\chi_{1}\left(P_{h, 1}\right)_{\text {out }}+O\left(h^{\infty}\right)$ so:

$$
\chi\left(P_{h}\right) e^{-i t P_{h} / h} \chi_{\text {out }}=\chi\left(P_{h, 1}\right) e^{-i t P_{h, 1} / h} \chi_{\text {out }}+O\left(h^{\infty}\right),
$$

which is the desired result.

\section{A. Appendix 1}

In this section, we prove that generalized many-body Schrödinger operators satisfy the hypotheses $(H)$, as indicated in Example 2, Sect. 2.

We first review the definition of generalized many-body Schrödinger operators introduced by Agmon [Ag]. One considers a finite dimensional real vector space $X$ with a positive definite quadratic form, and a finite family $\left\{X_{a}\right\}, a \in A$ of linear vector subspaces of $X$ closed under intersection and obeying $\bigcap_{a \in \mathscr{A}} X_{a}=\{0\}$ and $X \in\left\{X_{a}\right\}$. One denotes by $X^{a}$ the space $X_{a}^{\perp}$, by $\pi^{a}, \pi_{a}$ the orthogonal projections on $X^{a}$ and $X_{a}$.

On $\mathscr{A}$ one puts a partial ordering by saying that $b \subset a$ if $X^{b} \subset X^{a}$. With this ordering one gets that $X_{a_{\max }}=\{0\}$. Let as before $D_{x}=\frac{1}{i} \partial_{x}$ and let $\langle x\rangle=(1+g(x, x))^{1 / 2}$.

One defines a many-body Schrödinger operator by:

$$
P_{h}=g^{\prime}\left(h D_{x}, h D_{x}\right)+V(x),
$$

where: $V(x)=\sum_{a \in \mathscr{A}} V_{a}\left(\pi^{a} x\right)$ and $g^{\prime}$ is the dual quadratic form of $g$.

We assume that $V_{a} \in C^{\infty}\left(X^{a}, \mathbb{R}\right)$ satisfies:

$$
\text { (H.1) }\left|D_{y}^{\alpha} V_{y}(y)\right| \leqq C_{\alpha}\langle y\rangle^{-\rho-|\alpha|} \text { for } \rho>0, \quad \alpha \in \mathbb{N}^{\operatorname{dim} X^{a}} \text {. }
$$

From now on we can as well assume that $(X, g)$ is $\mathbb{R}^{n}$ with the usual metric and $P_{h}=-h^{2} \Delta+V(x)$. We will denote by $p(x, \xi)=\xi^{2}+V(x)$ the symbol of $P_{h}$.

The generalization of regular many-body Schrödinger operators introduced above allows for example to add $k$-body forces or exterior fields without changing 
the nature of the problem. The many-body structure of the potential energy term is most naturally described by the use of the following metric on $T^{*}(X)$ :

$$
g_{x, \xi}(d x, d \xi)=\frac{(d \xi)^{2}}{\langle\xi\rangle^{2}}+\sum_{a \in \mathscr{A}} \frac{\left(\pi^{a} d x\right)^{2}}{\left\langle x^{a}\right\rangle^{2}} .
$$

Let us denote it by $g$ for simplicity. Then with the definitions of Sect. 2, we get that $p(x, \xi)$ is in the symbol class $S\left(\langle\xi\rangle^{2}, g\right)$. To describe the behaviour of a classical many-body system near infinity, one has to consider its subhamiltonians, which are defined as follows:

for $a \in \mathscr{A}, a \neq a_{\max }$, we set:

$$
p^{a}\left(x^{a}, \xi^{a}\right)=\left(\xi^{a}\right)^{2}+\sum_{b \subset a} V_{b}\left(x^{b}\right) \stackrel{\text { def }}{=}\left(\xi^{a}\right)^{2}+V^{a}\left(x^{a}\right) .
$$

The energy levels which are trapping for a subhamiltonian are the exact classical analogs of the thresholds for the quantum Hamiltonian $\boldsymbol{P}_{\boldsymbol{h}}$. We have the following proposition, which was proven for in the case of three-particle Hamiltonians by Gérard [Ge1] and in the general case by Wang [Wa].

Proposition A.1. Let $p(x, \xi)$ be a classical many-body Hamiltonian and let $I \subset \mathbb{R}$ an energy interval which is non-trapping for all $p^{a}, a \neq a_{\max }$, and such that $0 \notin I$.

Then there exist a function $G(x, \xi) \in C^{\infty}\left(T^{*}\left(\mathbb{R}^{n}\right)\right)$ and constants $C_{0}, C_{1}, \varepsilon_{0}$ such that:

$$
\begin{gathered}
H_{p} G(x, \xi) \geqq C_{0}>0 \quad \text { on } \quad p^{-1}(I) \cap\left\{|x| \geqq C_{1}\right\} . \\
G=x \cdot \xi+r(x, \xi),
\end{gathered}
$$

where $r(x, \xi) \in S\left(\langle\xi\rangle^{-\infty}, g\right)$.

If $I$ is also non-trapping for $p$, then one has moreover:

$$
H_{p} G(x, \xi) \geqq C_{0}>0 \quad \text { on } \quad p^{-1}(I) .
$$

Note that the condition $0 \notin I$ is equivalent to $I$ non-trapping for $p_{a_{\min }}(x, \xi)=\xi^{2}$.

Finally we prove that one can modify the potential $V(x)$ on a compact set to make $I$ non-trapping, so that $p$ satisfies hypothesis $(H .7)$.

Lemma A.2. Let $p(x, \xi)$ a many-body classical Hamiltonian and I an energy interval such that $I$ is non-trapping for all $p^{a}, a \neq a_{\max }$. Let $G(x, \xi)$ be the escape function of Proposition A.1. Then there exists a function $W(x) \in C_{0}^{\infty}(X)$ such that if $\tilde{p}=p+W$ one has:

$$
H_{\tilde{p}} G \geqq C_{0} \quad \text { on } \quad \tilde{p}^{-1}(I) .
$$

Proof. By a covering argument, it suffices to consider the case when $I$ is a small neighborhood of an energy level $\lambda_{0}$. We choose a function $\chi \in C_{0}^{\infty}\left(\mathbb{R}^{+}\right)$with $\chi^{\prime} \leqq 0$, such that $\chi(s)=1$ for $0 \leqq s \leqq 1 / 2, \chi(2 / 3)=1 / 2, \chi(s)=0$ for $s \geqq 1$.

We will choose $W(x)=C_{1} \chi\left(\frac{|x|}{C_{2}}\right)$ for some constants $C_{1}, C_{2}$ to be chosen later.

Recall that given any $\varepsilon>0$, the escape function $G$ can be chosen such that $H_{p} G \geqq-\varepsilon$ everywhere (see [Wa, Theorem 2.2]) and that $G=x \cdot \xi+r$, with $r \in S\left(\langle\xi\rangle^{-\infty}, g\right)$. 
We first notice that if $\left|\tilde{p}-\lambda_{0}\right| \leqq \frac{\varepsilon_{1}}{2}$, one has $|x| \geqq \frac{2}{3} C_{2}$, as soon as $C_{1} \geqq C_{1}\left(\lambda_{0}, V, \varepsilon_{1}\right)$, since in $\{s \leqq 2 / 3\}$ one has $\chi(s) \geqq 1 / 2$.

We also know that $H_{p} G \geqq C_{0}$ in $|p-\lambda| \leqq \varepsilon_{1}$, and $|x| \geqq C_{0}$. If $|x| \geqq\left(1-\varepsilon_{0}\right) C_{2}, \varepsilon_{0}$ depending only on $\varepsilon_{1}$, and if $|\tilde{p}-\lambda| \leqq \frac{\varepsilon_{1}}{2}$, one has $|p-\lambda| \leqq \varepsilon_{1}$, so $H_{p} G \geqq C_{0}$. Also:

$$
H_{\tilde{p}} G=H_{p} G-\frac{C_{1}}{C_{2}}|x| \chi^{\prime}\left(\frac{|x|}{C_{2}}\right)-\frac{C_{1}}{C_{2}} \chi^{\prime}\left(\frac{|x|}{C_{2}}\right) \frac{x}{|x|} \cdot \frac{\partial r}{\partial \xi} \geqq \frac{C_{0}}{2},
$$

if we take $C_{1} / C_{2}$ large enough.

So it remains to consider the region:

$$
|\tilde{p}-\lambda| \leqq \frac{\varepsilon_{1}}{2}, \text { and } \frac{2}{3} C_{2} \leqq|x| \leqq\left(1-\varepsilon_{0}\right) C_{2} .
$$

In this region we have:

$$
-\frac{C_{1}}{C_{2}}|x| \chi^{\prime}\left(\frac{|x|}{C_{2}}\right) \geqq C_{1} \varepsilon_{2}\left(\varepsilon_{0}\right) .
$$
Since $H_{p} G \geqq-\varepsilon$, with $\varepsilon$ small fixed, by taking $C_{1}>C_{1}\left(C_{0}, \lambda_{0}, V, \varepsilon\right)$, and $\frac{C_{1}}{C_{2}}$ large
enough we get:

$$
H_{\tilde{p}} G \geqq \frac{C_{0}}{2} \quad \text { in } \quad|\tilde{p}-\lambda| \leqq \frac{\varepsilon_{1}}{2}, \quad \frac{2}{3} C_{2} \leqq|x| \leqq\left(1-\varepsilon_{0}\right) C_{2}
$$

This completes the proof of the lemma.

\section{B. Appendix 2}

In this section we collect some technical computations. In the statement of the first proposition we use the notations of the proof of Theorem 3.7.

Proposition B.1. Let us put $a(h)=\Gamma(h) / h$. Then one has:

$$
K_{n}(a, t)=\int_{0}^{t} \frac{O\left(h^{\infty}\right) e^{-(t-s) a}}{\langle s\rangle^{n}} d s=O\left(h^{\infty}\right)\left(\frac{1}{a\langle t\rangle^{n}}+e^{-a t}\right)
$$

uniformly for $h \leqq \varepsilon_{0}$ and $t \geqq 0$.

Proof. We have to estimate:

$$
I_{n}(a, t)=\int_{0}^{t} \frac{e^{a s}}{\langle s\rangle^{n}} d s
$$

uniformly for $0<h<\varepsilon_{0}$ and for $t \geqq 0$.

Let us consider different cases:

Case $A$ : $t \geqq C_{1}$ for some $C_{1}>1$. Then:

$$
I_{n} \leqq C_{n} \int_{0}^{t} e^{a s} d s+C_{n} \int_{1}^{t} \frac{e^{a s}}{|s|^{n}} d s \leqq a^{-1}\left(e^{C_{1 a}}-1\right)+C_{n} \int_{C_{1}}^{t} e^{a s} s^{-n} d s .
$$


Let us denote by $J_{n}$ the last integral. We have:

$$
J_{n}=a^{n-1} \int_{a}^{a t} \frac{e^{s}}{s^{n}} d s
$$

We consider now different subcases:

Case $A .1: C_{0}^{-1} \leqq a \leqq C_{0}$ for some $C_{0}>0$.

Let $s_{n}$ be such that for $s \geqq s_{n}$ one has:

$$
\left(s^{-n} e^{s}\right)^{\prime}=s^{-n} e^{s}-n s^{-n+1} e^{s} \geqq \frac{1}{2} s^{n} e^{s} .
$$

We can write:

$$
\int_{a C_{1}}^{a t} e^{s} s^{-n} d s=\int_{a C_{1}}^{s_{n}} e^{s} s^{-n} d s+\int_{s_{n}}^{a t} e^{s} s^{-n} d s
$$

Since $a$ is bounded below, we can always assume that $a C_{1} \geqq s_{n}$, by taking $C_{1}$ large enough, so we just have to consider the last integral. By (B.1) it is bounded by:

$$
C_{n}\left((a t)^{-n} e^{a t}-C_{n}^{\prime}\right)
$$

So we get that:

$$
I_{n}(a, t) \leqq \frac{e^{C_{n} a}-1}{a}+C_{n} \frac{e^{a t}}{a t^{n}} .
$$

So finally using that $C_{0}^{-1} \leqq a \leqq C_{0}$, we get:

$$
K_{n}(a, t) \leqq O\left(h^{\infty}\right)\left(\frac{1}{a t^{n}}\right) .
$$

Case A.2: $a=o_{h}(1)$. We have again to compute $J_{n}$. We have to consider two subcases:

Case A.2i): at $\leqq s_{n}$.

Then we have:

$$
J_{n} \leqq C_{n} a^{n-1} \int_{a}^{a t} s^{-n} d s \leqq C_{n} t^{-n}
$$

So we get finally:

$$
K_{n}(a, t) \leqq O\left(h^{\infty}\right) e^{-a t}\left(\frac{e^{C_{1} a}-1}{a}\right)+\frac{e^{-a t}}{t^{n-1}} \leqq \frac{C_{n}}{a t^{n}}
$$

since $a t \leqq s_{n}$ and $a^{-1}\left(e^{c_{1} a}-1\right)$ is bounded.

Case A.2ii): at $\geqq s_{n}$. Then:

$$
J_{n} \leqq C_{n} a^{-n+1} \int_{s_{n}}^{a t} e^{s} s^{-n} d s .
$$

By (B.1), the last integral is bounded by $C_{n}(a t)^{-n} e^{a t}$. So we get finally:

$$
K_{n}(a, t) \leqq O\left(h^{\infty}\right)\left(\frac{C_{n}}{a t^{n}}+C_{n} e^{a t}\right)
$$


Case A.3: $a^{-1}=o_{h}(1)$.

Then for $h$ small enough $J_{n}$ can be estimated using (B.1) since $a \geqq s_{n}$, and we get:

$$
K_{n}(a, t) \leqq O\left(h^{\infty}\right)\left(e^{-a t}\left(\frac{e^{C_{1} a}-1}{a}\right)+\frac{C_{n}}{a t^{n}}\right) .
$$

Let us now consider the case when $t$ is bounded.

Case $B: t \leqq C_{1}$ for some $C_{1}>1$. Then:

$$
K_{n}(a, t) \leqq C_{n} O\left(h^{\infty}\right) \int_{0}^{t} e^{a(s-t)} d s \leqq C_{n} a^{-1}\left(1-e^{-a t}\right) .
$$

Since $t \leqq C_{1}$, we get that:

$$
K_{n}(a, t) \leqq \frac{O\left(h^{\infty}\right)}{a} .
$$

It is now straightforward to check that in all cases $K_{n}$ is bounded by:

$$
O\left(h^{\infty}\right)\left(\frac{1}{a\langle t\rangle^{n}}+e^{-a t}\right)
$$

which completes the proof of the Proposition.

We now give a computation used in the proof of Theorem 3.8. As above we denote by $a(h)$ the width $\Gamma(h) / h$. We use the notations in the proof of Theorem 3.8 .

Proposition B.2. If $a(h)=O_{h}(1)$ then:

$$
\left\|r_{\alpha}(t)\right\| \leqq C e^{-a t(1-\alpha)}\left(1+\alpha^{-1 / 2}\left(1-e^{-c a t}\right)^{1 / 2}\right) .
$$

If $a(h)^{-1}=o_{h}(1)$ then:

$$
\left\|r_{\alpha}(t)\right\| \leqq C a^{-1} e^{-a t(1-\alpha)} .
$$

Proof. The estimate on $J(t, h)=\left\|r_{\alpha}(t)\right\|$ when $a^{-1}$ is $o_{h}(1)$ is obvious. Let us now consider the case when $a=O_{h}(1)$ and for simplicity of notations replace $P_{h, 1}$ by $P_{h}$. We write: $J(t, h)^{2}=e^{-2 t a} I(t, h)$, where:

$$
I(t, h)=\int_{0}^{\alpha t} d s \int_{0}^{\alpha t} d s^{\prime}\left(e^{-i s\left(P_{h}-E\right) / h} \chi\left(P_{h}\right) \chi_{\text {out }} u_{h}, e^{-i s^{\prime}\left(P_{h}-E\right) / h} \chi\left(P_{h}\right) \chi_{\text {out }} u_{h}\right) .
$$

Let us put $\operatorname{Re} E=E_{0}$. Then:

$$
\begin{array}{r}
\left(e^{-i s\left(P_{h}-E\right) / h} \chi\left(P_{h}\right) \chi_{\text {out }} u_{h}, e^{-i s^{\prime}\left(P_{h}-E\right) / h} \chi\left(P_{h}\right) \chi_{\text {out }} u_{h}\right) \\
=e^{a\left(s+s^{\prime}\right)}\left(\chi_{\text {out }}^{*} e^{-i\left(s-s^{\prime}\right)\left(P_{h}-E_{0}\right)} \chi^{2}\left(P_{h}\right) \chi_{\text {out }} u_{h}, u_{h}\right) .
\end{array}
$$

We put $s+s^{\prime}=\sigma$ and $s-s^{\prime}=\sigma^{\prime}$. Then:

$$
I(t, h)=\sqrt{2} \iint_{\Omega} e^{a \sigma}\left(\chi_{\text {out }}^{*} e^{-i \sigma^{\prime}\left(P_{h}-E_{0}\right) / h} \chi^{2}\left(P_{h}\right) \chi_{\text {out }} u_{h}, u_{h}\right) d \sigma d \sigma^{\prime},
$$

where $\Omega$ is the region drawn on Fig. 1 .

Let $\sigma_{0}$ be a time such that no trajectory starting from supp $\chi_{\text {out }}$ is still in supp $\chi_{\text {out }}$ for $t \geqq \sigma_{0}$. Let us first assume that $\alpha t \geqq \sigma_{0}$. Then by the results of Sect. 7, we know 


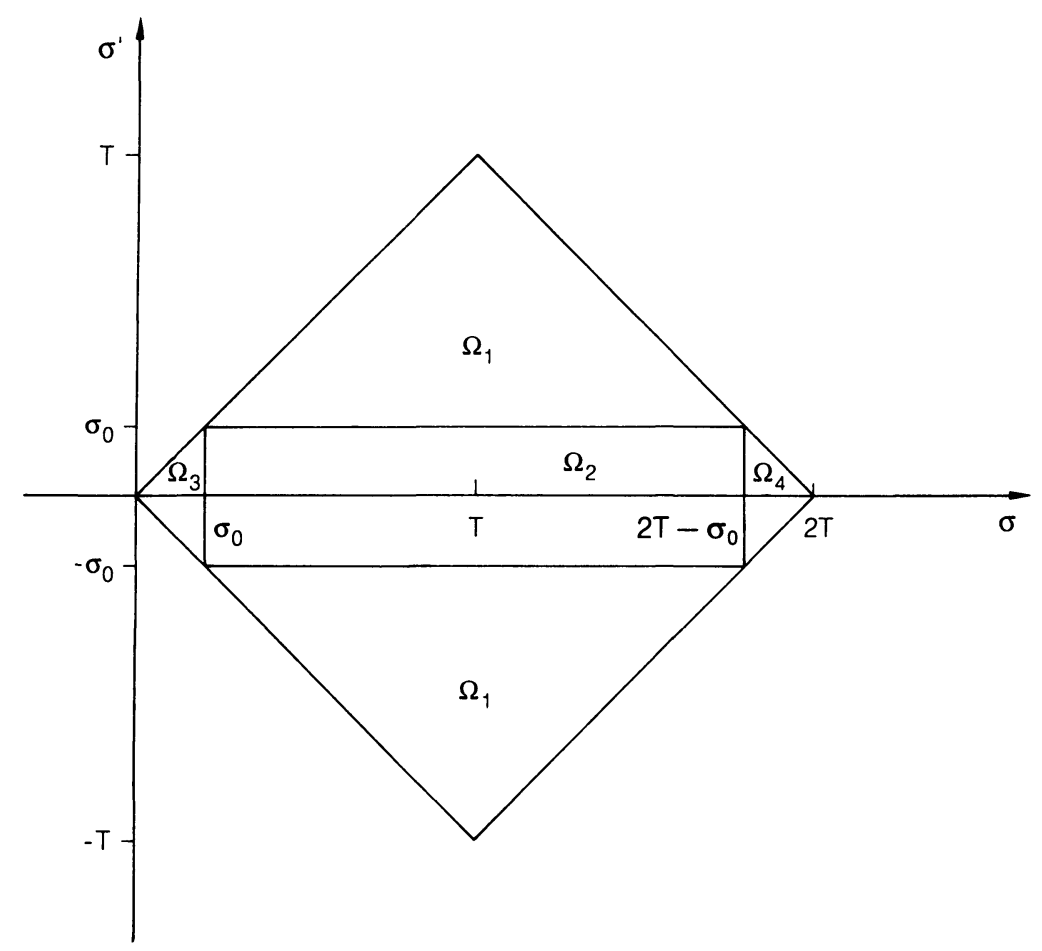

Fig. 1.

that for $s \geqq \sigma_{0}$, one has:

$$
\left\|\chi_{\text {out }}^{*} e^{-i t\left(P_{h}-E_{0}\right) / h} \chi^{2}\left(P_{h}\right) \chi_{\text {out }}\right\|=O\left(\frac{h^{\infty}}{\langle t\rangle^{\infty}}\right) .
$$

Accordingly we cut $\Omega$ in four pieces as shown in Fig. 1.

Let us now compute the various integrals.

Integral on $\Omega_{1}$ : We integrate first in $\sigma^{\prime}$ and get an integral which is $O\left(h^{\infty}\right)$ by the previous remark. Then the integral in $\sigma$ gives a term which is $O\left(h^{\infty}\right)\left(a^{-1} e^{2 a \alpha t}-1\right)$.

Integral on $\Omega_{2}$ : The integral is not better than $O(1)$, so we get a term equal to:

$$
\left.O\left(a^{-1}\right)\left(e^{a\left(2 \alpha t-s_{0}\right.}\right)-e^{a s_{0}}\right) \text {. }
$$

Integral on $\Omega_{3}$ : We integrate first in $\sigma^{\prime}$ and get a term which is $O(\sigma)$. Then the integral in $\sigma$ gives: $O\left(a^{-1}\left(e^{a s o}-1\right)\right)$.

Integral on $\Omega_{4}$ : We integrate first in $\sigma^{\prime}$ and get a term $O(2 \alpha t-\sigma)$. Then the integral in $\sigma$ gives a term which is:

$$
O\left(a^{-1} e^{2 a \alpha t}\left(1-e^{-a s_{0}}\right)\right)
$$

Let us now estimate $e^{-2 a t} I(t, h)$. If we denote by $A_{i}$ the term coming from the 
integral on $\Omega_{i}$, we see that $A_{1}$ can be absorbed into $A_{2}$ and we get:

$$
\begin{aligned}
& A_{2}=O\left(a^{-1}\right)\left(e^{-a\left(s_{0}+2 t(1-\alpha)\right)}-e^{a\left(s_{0}-2 t\right)}\right), \\
& A_{3}=O\left(a^{-1}\right) e^{-2 a t}\left(e^{a s_{0}}-1\right), \\
& A_{4}=O\left(a^{-1}\right) e^{-2 a t(1-\alpha)}\left(1-e^{-a s_{0}}\right) .
\end{aligned}
$$

We will now find a simpler upper bound by considering separately the two cases $C_{1} \leqq a$ for some constant $C_{1}$ and $a=o_{h}(1)$.

Case 1: Then one gets easily that for $t \geqq T_{0}, J(t, h)^{2}$ is $O\left(a^{-1} e^{-2 a t(1-\alpha)}\right)$.

Case 2. : We use that if $a=o_{h}(1)$ then $a^{-1}\left(1-e^{ \pm s_{0} a}\right)$ is $O_{h}(1)$. We find that $A_{i}$ for $i=1,3,4$ are $O\left(e^{-2 a t(1-\alpha)}\right)$. For $A_{2}$ we get:

$$
\left.A_{2} \leqq C a^{-1} e^{-2 a t(1-\alpha)}\left(\left|e^{-a s_{0}}-1\right|+\mid 1-e^{a\left(s_{0}-\alpha t\right.}\right) \mid\right) .
$$

By the previous remark this completes the proof of the proposition.

\section{Appendix 3}

In this section, we will prove Proposition 3.5. We will prove the following lemma, which implies directly Proposition 3.5.

Lemma C.1. Let $u_{h}$ be a quasiresonant state. Then $u_{h}$ satisfies the following properties:

i) $\forall C_{1}>0 \exists R_{1} \quad$ such that $F S u_{h} \cap\left\{|x| \geqq R_{1}\right\} \subset\left\{(x, \xi) \mid G(x, \xi) \geqq C_{1}\right\}$.

If moreover:

$$
F S u_{h} \cap \operatorname{sing} \operatorname{supp} V_{s}=\varnothing
$$

then the following properties hold:

ii) $F S u_{h} \subset p^{-1}(I)$,

iii) $F S u_{h} \subset \Gamma_{+} \Leftrightarrow \forall C_{1} \gg 1, \quad F S u_{h} \cap\left\{G(x, \xi) \leqq-C_{1}\right\}=\varnothing$,

iv) $F S u_{h} \cap\{|x| \geqq R\} \subset\left\{G(x, \xi) \geqq C_{1}\right\} \Rightarrow F S u_{h} \cap\{|x| \geqq R\} \subset \Gamma_{+}$

v) $F S u_{h} \subset \Gamma_{+} \Leftrightarrow \forall R \geqq 1, \quad F S u_{h} \cap\{|x| \geqq R\} \subset \Gamma_{+}$.

Proof. Proof of i): Let $\left(x_{0}, \xi_{0}\right) \in F S u_{h} \cap\left\{|x| \geqq R_{1}\right\}$, for some $R_{1} \geqq 1$. We choose some $R_{0} \geqq 1$ such that $H_{p} G \geqq c_{0}$ in $\left\{|x| \geqq R_{0}\right\} \cap p^{-1}(I)$ and $V_{s}=0$ in $\left\{|x| \geqq R_{0}\right\}$. Then we know that for $-T_{0} \leqq t \leqq 0$, where $T_{0}=\varepsilon_{0}\left(R_{1}-R_{0}\right) . \phi_{t}\left(x_{0}, \xi_{0}\right)$ stays in $\left\{|x| \geqq R_{0}\right\}$, and hence in $F S u_{h}$ by Theorem 3.2. So we have $G\left(\phi_{T_{0}}\left(x_{0}, \xi_{0}\right)\right) \geqq C_{0}$, by Definition 3.4. Since $H_{p} G \geqq c_{0}$ along the trajectory, we get that $G\left(x_{0}, \xi_{0}\right) \geqq C_{0}+c_{0} \varepsilon_{0}\left(R_{1}-R_{0}\right)$. This can be made bigger than $C_{1}$ by choosing $R_{1}$ big enough, which proves $\mathrm{i})$.

Proof of ii): Let $\left(x_{0}, \xi_{0}\right) \in T^{*}\left(\mathbb{R}^{n}\right) \cap F S u_{h}$. If $x_{0} \in \operatorname{supp} V_{s}$, then $\left(x_{0}, \xi_{0}\right) \notin F S u_{h}$ by hypothesis (C.1). If $x_{0} \notin \operatorname{supp} V_{s}$, then $u_{h}$ solves:

$$
\left(-h^{2} \Delta+V-E(h)\right) u_{h}=O\left(h^{\infty}\right)
$$

microlocally near $\left(x_{0}, \xi_{0}\right)$, and $E(h) \rightarrow \lambda_{0}$ with $\lambda_{0} \in \dot{I}$, so by ellipticity one has $\left(x_{0}, \xi_{0}\right) \in p^{-1}(I)$. 
Proof of iii): $\Rightarrow$ : This follows from the fact that $\exists C_{0}$ such that $\Gamma_{+} \subset\left\{G(x, \xi) \geqq-C_{0}\right\}$ (see [Ge-Sj, Appendix]).

$\Leftarrow:$ Let $\left(x_{0}, \xi_{0}\right) \in T^{*}\left(\mathbb{R}^{n}\right) \cap F S u_{h}$. If $x_{0} \in \operatorname{supp} V_{s}$, then $\left(x_{0}, \xi_{0}\right) \notin F S u_{h}$ by hypothesis (C.1). So we can assume that $x_{0} \notin \operatorname{supp} V_{s}$, and $\left(x_{0}, \xi_{0}\right) \in p^{-1}(I)$ by ii). Suppose that $\left(x_{0}, \xi_{0}\right) \notin \Gamma_{+}$. Since outside the set of trapped trajectories $K, d p$ is non-zero, and since $\Gamma_{+}$is $H_{p}$ invariant, $d p$ is non-zero along the whole $H_{p}$ trajectory starting from $\left(x_{0}, \xi_{0}\right)$. We consider two different cases:

Case 1: There exists a minimal time $T$ such that $\pi_{x} \phi_{T}\left(x_{0}, \xi_{0}\right) \in \operatorname{supp} V_{s}$. We will then say that $\left(x_{0}, \xi_{0}\right)$ is a point of type 1 . Then by hypothesis (C.1), we know that $\phi_{T+\varepsilon}\left(x_{0}, \xi_{0}\right) \notin F S u_{h}$ for $\varepsilon$ small enough. Since $u_{h}$ solves (C.2) along the $H_{p}$ trajectory between $\phi_{T+\varepsilon}\left(x_{0}, \xi_{0}\right)$ and $\left(x_{0}, \xi_{0}\right)$, we can apply Theorem 3.2 and get that $\left(x_{0}, \xi_{0}\right) \notin F S u_{h}$.

Case 2: The $H_{p}$ trajectory starting backwards from $\left(x_{0}, \xi_{0}\right)$ does not meet $\operatorname{supp} V_{s}$. We will then say that $\left(x_{0}, \xi_{0}\right)$ is a point of type 2 . Then since $\left(x_{0}, \xi_{0}\right) \notin \Gamma_{+}$, we get easily that $G\left(\phi_{t}\left(x_{0}, \xi_{0}\right)\right) \rightarrow-\infty$ when $t \rightarrow-\infty$. Applying again Theorem 3.2, we get that if $\left(x_{0}, \xi_{0}\right) \in F S u_{h}$ then there exists some points in $F S u_{h} \cap\left\{G \leqq-C_{1}\right\}$ for $C_{1} \geqq 1$, which gives a contradiction.

Proof of iv): Let $\left(x_{0}, \xi_{0}\right) \in F S u_{h} \cap\{|x| \geqq R\}$ and $\left(x_{0}, \xi_{0}\right) \notin \Gamma_{+}$. If $\left(x_{0}, \xi_{0}\right)$ is a point of type 1 , then $\left(x_{0}, \xi_{0}\right) \notin F S u_{h}$ by the same argument as above. If $\left(x_{0}, \xi_{0}\right)$ is of type 2 , then since $\left(x_{0}, \xi_{0}\right) \notin \Gamma_{+}$, one knows that $\phi_{t}\left(x_{0}, \xi_{0}\right) \rightarrow \infty, G\left(\phi_{t}\left(x_{0}, \xi_{0}\right)\right) \rightarrow-\infty$, when $t \rightarrow-\infty$. By the same argument as in the proof of iii), we get that there would exist a point $\phi_{t}\left(x_{0}, \xi_{0}\right) \in F S u_{h} \cap\left\{(x, \xi)|| x \mid \geqq R, G(x, \xi) \geqq C_{1}\right.$, for any $R, C_{1}$. This gives a contradiction and proves iv).

Proof of v): $\Rightarrow$ is obvious. To prove $\epsilon$, it suffices as above to consider a point $\left(x_{0}, \xi_{0}\right) \in F S u_{h}$ of type 2 which does not belong to $\Gamma_{+}$. Since $\left(x_{0}\right) \notin \Gamma_{+}$, we know that $\phi_{t}\left(x_{0}, \xi_{0}\right) \rightarrow \infty$ when $t \rightarrow-\infty$. Using Theorem 3.2. and the fact that $\Gamma_{+}$is $H_{p}$ invariant, we get a contradiction. This completes the proof of the lemma.

Acknowledgements. The work on this problem began when both authors were visiting E.T.H. in Zürich. The authors are grateful to J. Fröhlich, W. Hunziker, H. Knörrer and E. Trubowitz for hospitality.

\section{References}

[A-M] Abraham, R., Marsden, J.: Foundations of Mechanics. New York: BenjaminCummings 1978

[Ag] Agmon, S.: Lectures on exponential decay of solutions of second order elliptic equations. Princeton, NJ: Princeton University Press 1982

[Ag-Co] Aguilar, J., Combes, J. M.: A class of analytic perturbations for one-body Schrödinger operators. Commun. Math. Phys. 22, 269-279 (1971)

[Ba1] Balslev, E.: Local spectral deformation techniques for Schrödinger operators. J. Funct. Anal. 29, 79-105 (1984)

[Ba2] Balslev, E.: Scattering theory of quantum mechanical three-body systems. Ann. I.H.P. 58, 125-160 (1980)

[Ba-Co] Balslev, E., Combes, J. M.: Spectral properties of many body Schrödinger operators with dilation analytic potentials. Commun. Math. Phys. 22, 280-294 (1971) 
[B-C-D] Briet, P., Combes, J. M., Duclos, P.: On the location of resonances of Schrödinger operators in the semiclassical limit I. Resonances free domains. J. Math. Anal. Appl., Vol. 126, (1) 90-99 (1987); II Barrier top resonances. Comm. P.D.E. 12, 201-222 (1987)

[C-D-K-S] Combes, J. M., Duclos, P., Klein, M., Seiler, R.: The shape resonance. Commun. Math. Phys. 110, 215-237 (1987)

[Cy] Cycon, H. L.: Resonances defined by modified dilations. Helv. Phys. Acta 58, 969-981 (1986)

[De] Derezinski, J.: Existence and analyticity of many-body scattering amplitudes at low energies. J. Math. Phys. 28, 1080-1088 (1989)

[Ge1] Gérard, C.: Semiclassical resolvent estimates for two and three-body Schrödinger operators. Comm. P.D.E. 15 (8), 1161-1178 (1990)

[Ge2] Gérard, C.: Asymptotique des pôles de la matrice de scattering pour deux obstacles strictement convexes. Bull. S.M.F. Mémoire $n^{\circ} 31$ tome 116 (1988)

[Ge-Ma2] Gérard, Ch., Martinez, A.: Principe d'absorption limite pour des opérateurs de Schrödinger a longue portée, C. R. Acad. Sci. Paris t.306, série I, 121-123 (1988)

[Ge-Ma1] Gérard, Ch., Martinez, A.: Prolongement méromorphe de la matrice de scattering pour des problèmes à deux corps à longue portée. Ann. l' I.H.P. 51, 81-110 (1989)

[Ge-Sj] Gérard, Ch., Sjöstrand, J.: Semiclassical resonances generated by a closed trajectory of hyperbolic type. Commun. Math. Phys. 108, 391-421 (1987)

[Gu-St] Guillemin, V., Sternberg, S.: Geometric asymptotics. A.M.S. Surveys, Vol. 14 (1977)

[Ha] Hagedorn, G.: A link between scattering resonances and dilation analytic resonances in few-body Quantum Mechanics. Commun. Math. Phys. 65, 181-188(1979)

[He] Helffer, B.: Semiclassical Analysis for the Schrödinger operator and applications. Lectures Notes in Mathematics Vol. 1336. Berlin, Heidelberg, New York: Springer 1988

[He-Ro] Helffer, B., Robert, D.: Calcul fonctionnel par la transformation de Mellin et opérateurs admissibles. J. Funct. Anal. 63, 246-268 (1983)

[He-Sj1] Helffer, B., Sjöstrand, J.: Multiple Wells in the semiclassical limit I. Comm. P.D.E. 9, 377-408 (1984)

[He-Sj2] Helffer, B., Sjöstrand, J.: Résonances en limite semiclassique. Bull. S.M.F. mémoire $\mathrm{n}^{\circ} 24 / 25$, tome 114 (1986)

[He-Sj3] Helffer, B., Sjöstrand, J.: Equation de Schrödinger avec champ magnétique et équation de Harper. Lectures Notes in Physics vol. 345, 118-197. Berlin, Heidelberg, New York: Springer 1989

[Hi-Si] Hislop, P., Sigal, I. M.: Shape resonances in quantum mechanics. Proceeding of the International Conference on Diff. Eq. in Math. Phys. Birmingham (Alabama) (1986)

[Hö] Hörmander, L.: The analysis of linear partial differential operators, Vols. III and IV, Berlin, Heidelberg, New York: Springer 1985

[Hu1] Hunziker, W.: Distortion analyticity and molecular resonances curves. Ann. Inst. Henri Poincaré 45, 339-358 (1986)

[Hu2] Hunziker, W.: Resonances, metastable states and exponential decay laws in Perturbation Theory. Commun. Math. Phys. 132 (1), 177-188 (1990)

[Ma] Martinez, A.: Estimations de l'effect tunnel pour le double puits II. Etats hautement excités. Bull. Soc. Math. France 116, 199-229 (1988)

[O] Orth, A.: Quantum mechanical resonance and limiting absorption: The many-body problem. Commun. Math. Phys. 126 (3), 559-573 (1990)

[Ro] Robert, D.: Autour de l'approximation semiclassique. Basel: Birkhaüser 1983

[S1] Sigal, I. M.: Complex transformations method and resonances in one-body quantum systems, Ann. Inst. Henri Poincaré 41, 103-114 (1984),

[S2] Sigal, I. M.: Sharp exponential bounds on the resonances... . Adv. Appl. Math.

[S3] Sigal, I. M.: Scattering theory for many-body systems. Lecture Notes in Mathematics, Vol. 1011. Berlin, Heidelberg, New York: Springer 1983

[S4] Sigal, I. M.: Analytic properties of the scattering matrix of many-particle systems. Int. Eq. Oper. Theory 9, 134-153 (1986)

[S-S] Sigal, I. M., Soffer, A.: Local Decay and propagation estimates for time dependent and time independent Hamiltonians. Preprint, Princeton University, 1988 
[Si1] Simon, B.: Resonances and complex scaling: A rigourous overview. Int. J. Quant. Chem. 14, 529-542 (1978)

[Si2] Simon, B.: The definition of molecular resonance curves by the method of exterior complex scaling. Phys. Lett. 71A, 211-214 (1979)

[Sj] Sjöstrand, J.: Semiclassical resonances generated by non-degenerate critical points, Proceedings Oberwolfach 1986. Lecture Notes in Mathematics Vol. 1256. Berlin, Heidelberg, New York: Springer 1986

[Sk1] Skibsted, E.: Truncated Gamow functions, $\alpha$-decay and the exponential decay law, Commun. Math. Phys. 104, 591-604 (1986)

[Sk2] Skibsted, E.: Truncated Gamow functions and the exponential decay law. Ann. Inst. H. Poincaré 46, 131-153 (1987)

[Sk3] Skibsted, E.: On the evolution of resonant states. J. Math. Anal. Appl. 141, 27-48 (1989)

[Wa] Wang, X. P.: Semiclassical resolvent estimates for $\mathrm{N}$-body Schrödinger operators, Preprint Technische Universität Berlin, 1989

Communicated by B. Simon 